\title{
Três Problemas em Complementaridade e Programação Matemática
}

\author{
Marcelo Gomes de Queiroz
}

TESE DE DOUTORADO APRESENTADA AO INSTITUTO DE MATEMÁTICA E ESTATÍSTICA DA UNIVERSIDADE DE SÃO PAULO

Curso: Doutorado em Ciência da Computação Orientador: Prof. Dr. Carlos Humes Júnior Co-orientador: Prof. Dr. Joaquim João Júdice (Universidade de Coimbra - Portugal)

São Paulo, 2001

O aluno recebeu apoio da FAPESP. 


\title{
Três Problemas em Complementaridade e Programação Matemática
}

\author{
Este exemplar corresponde à redação final da tese \\ apresentada por Marcelo Gomes de Queiroz, devida- \\ mente corrigida e aprovada pela comissão julgadora.
}

São Paulo, novembro de 2001.

Banca examinadora:

Prof. Dr. Carlos Humes Júnior IME - USP

Prof. Dr. José Mario Martínez IMECC - UNICAMP

Prof. Dr. Clóvis Caesar Gonzaga MTM - UFSC

Prof. Dr. Marcos Nereu Arenales ICMC - USP

Prof. Dr. Marco Antônio Brinati

IME - USP
IMECC - UNICAMP
MTM - UFSC
ICMC - USP
EP - USP




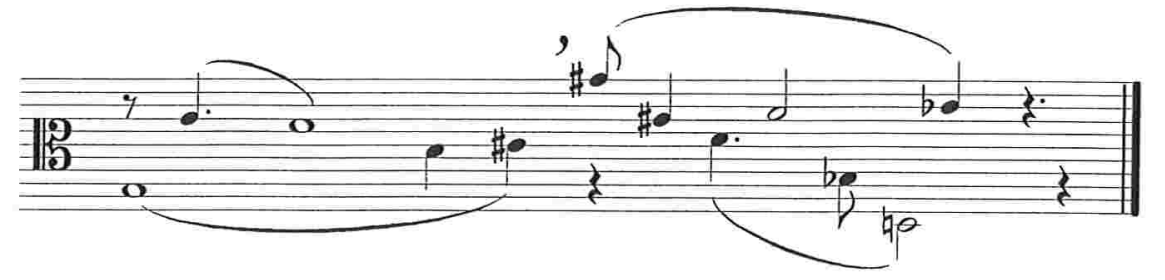




\section{Agradecimentos}

Aos meus pais

Ao Roberto

À Dilma

Ao Paulo

Ao Ernesto

Ao Carlos

Ao Joaquim 


\section{Resumo}

Este trabalho aborda três problemas que têm em comum modelagens envolvendo condições de complementaridade, e propostas de solução com ferramentas de programação matemática. As aplicações destes problemas incluem pesquisa operacional, regressão não-linear, redes neurais e equilíbrio de sistemas mecânicos. As técnicas de solução propostas combinam várias ferramentas de programação linear e não-linear, otimização combinatória e programação côncava. Testes numéricos estão incluidos.

\footnotetext{
Abstract

This work focuses on three problems that share models involving complementarity conditions and solution methods using mathematical programming tools. The applications include operational research, nonlinear regression, neural networks and equilibrium of mechanical systems. The proposed solution methods combine several tools from linear and nonlinear programming, combinatorial optimization and concave programming. Numerical tests are included.
} 


\section{Sumário}

Lista de Definições $\quad$ xiii

Lista de Teoremas, Proposições e Corolários $\quad$ xv

Lista de Algoritmos e Heurísticas $\quad$ xix

$\begin{array}{ll}\text { Notações } & \text { xxi }\end{array}$

Glossário $\quad$ xxiii

Introdução $\quad$ xxv

1 O Problema de Ajuste de Dobradiças 1

1.1 O Problema do Ajuste de Funções Dobradiças . . . . . . . . . . . . 5

1.2 O Algoritmo para Obtenção de Dobradiça (HFA) . . . . . . . . . 18

1.3 Um Método Enumerativo para o (HF) . . . . . . . . . . . . . . 23

1.4 O Problema de Ajuste Linear de Dobradiças . . . . . . . . . . . . . 33

1.5 Experiência Computacional . . . . . . . . . . . . . . 39

1.6 Conclusão e Trabalho Futuro . . . . . . . . . . . . . . . . 45

2 O Problema de Valores Próprios Complementar Simétrico $\quad 47$

2.1 Problemas de Valores Próprios e Otimização . . . . . . . . . . . . . 51 
2.2 O Problema de Valores Próprios Complementar . . . . . . . . . . 55

2.3 O Problema de Valores Próprios Complementar Simétrico . . . . . . . 60

2.4 Experiência Computacional . . . . . . . . . . . . . 75

2.5 Conclusão e Trabalho Futuro . . . . . . . . . . . . . . . . 77

Apêndice: Caso Assimétrico . . . . . . . . . . . . . . . . . . . . . . . . . . 79

3 Cortes Profundos Para o LCP 83

3.1 Cortes de Concavidade e o Método de Tuy . . . . . . . . . . . . . 86

3.2 Os Métodos de Tuy e Thoai \& Tuy Para o LCP . . . . . . . . . . . . 110

3.3 Cortes Profundos para o LCP . . . . . . . . . . . . . . . . . . 117

3.4 Experiência Computacional . . . . . . . . . . . . . . . . . 129

3.5 Conclusão e Trabalho Futuro . . . . . . . . . . . . . . . . 134

4 Conclusão Geral $\quad 137$ 


\section{Lista de Definições}

Problema de Ajuste Euclideano de Dobradiças - HF $\ldots \ldots \ldots \ldots \ldots \ldots \ldots \ldots \ldots$

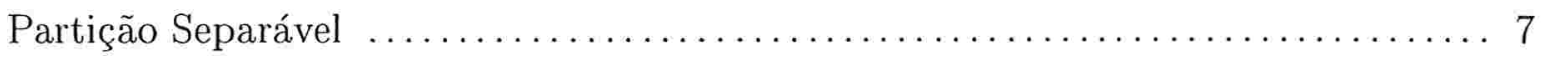

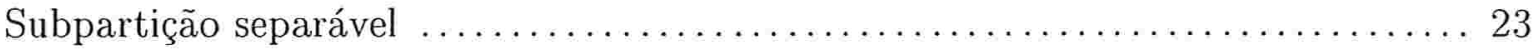

Problema de Ajuste Linear de Dobradiças - LHF $\ldots \ldots \ldots \ldots \ldots \ldots \ldots \ldots \ldots \ldots \ldots \ldots$

Problema Clássico de Valores Próprios - EiP $\ldots \ldots \ldots \ldots \ldots \ldots \ldots \ldots \ldots \ldots \ldots \ldots \ldots \ldots \ldots \ldots \ldots \ldots$

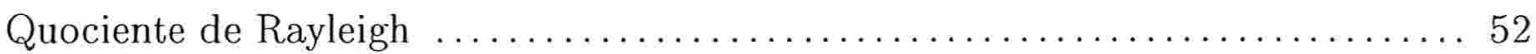

Problema de Valores Próprios Geral - GEiP $\ldots \ldots \ldots \ldots \ldots \ldots \ldots \ldots \ldots \ldots \ldots . \ldots \ldots$

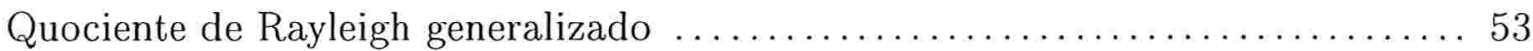

Problema de Valores Próprios Complementar - EiCP .................. 55

Problema de Valores Próprios Complementar Geral $\ldots \ldots \ldots \ldots \ldots \ldots \ldots \ldots \ldots$

Problema de Programação Côncava - PPC $\ldots \ldots \ldots \ldots \ldots \ldots \ldots \ldots \ldots \ldots \ldots \ldots \ldots \ldots \ldots \ldots \ldots \ldots$

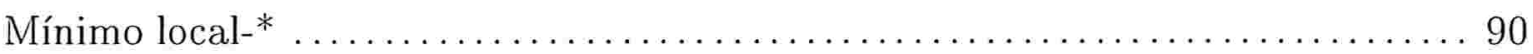

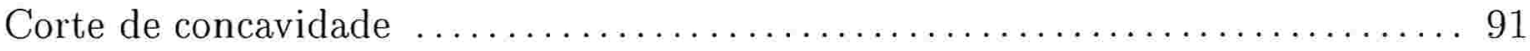

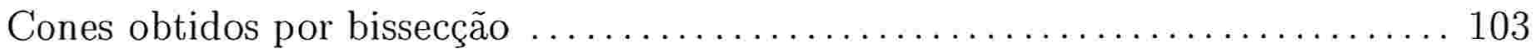

Problema de Complementaridade Linear - LCP $\ldots \ldots \ldots \ldots \ldots \ldots \ldots \ldots \ldots \ldots \ldots \ldots \ldots \ldots \ldots$

LCP como Problema de Programação Côncava $\ldots . \ldots \ldots \ldots \ldots \ldots \ldots \ldots \ldots \ldots \ldots \ldots \ldots$

LCP como PPC Paramétrico - LCP- $\alpha \beta \ldots \ldots \ldots \ldots \ldots \ldots \ldots \ldots \ldots \ldots \ldots \ldots \ldots \ldots \ldots$ 


\section{Lista de Teoremas, Proposições e}

\section{Corolários}

Lema: Partições separáveis são estritamente separáveis $\ldots \ldots \ldots \ldots \ldots \ldots \ldots \ldots 7$

Lema: Partições separáveis e cones de complementaridade $\ldots \ldots \ldots \ldots \ldots \ldots \ldots . . \ldots$

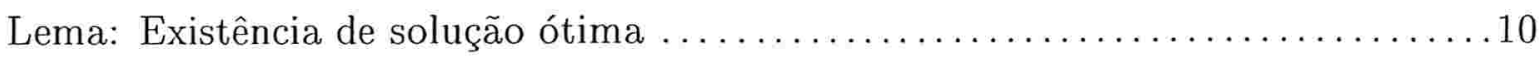

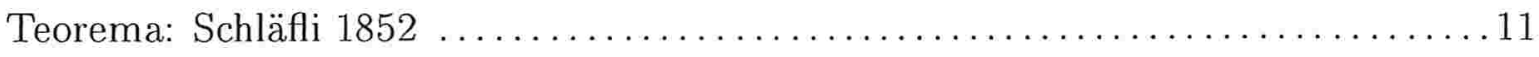

Lema: Partições não-separáveis geram problemas degenerados $\ldots \ldots \ldots \ldots \ldots \ldots 16$

Lema: Breiman 1993 ............................................

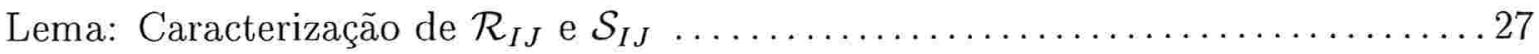

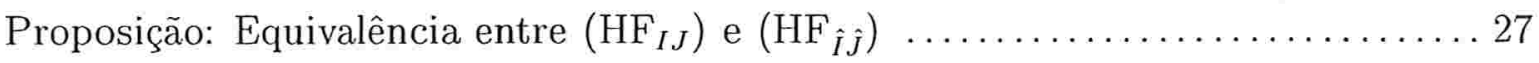

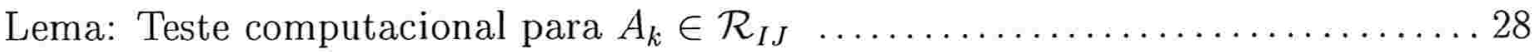

Lema: Hereditariedade de solução ótima de $\left(L B_{I J}\right) \ldots \ldots \ldots \ldots \ldots \ldots \ldots \ldots \ldots \ldots \ldots \ldots \ldots \ldots \ldots$

Proposição: Corretude e complexidade do método Enumerativo $\ldots . \ldots \ldots \ldots \ldots . \ldots 33$

Lema: (EiP) como problema de otimização $\ldots \ldots \ldots \ldots \ldots \ldots \ldots \ldots \ldots \ldots \ldots \ldots \ldots \ldots \ldots \ldots \ldots \ldots \ldots$

Lema: (GEiP) como problema de otimização $\ldots \ldots \ldots \ldots \ldots \ldots \ldots \ldots \ldots \ldots \ldots \ldots \ldots \ldots \ldots \ldots \ldots$

Lema: Homogeneidade de $\lambda(\cdot)$ e ortogonalidade de $\nabla \lambda(\cdot) \ldots \ldots \ldots \ldots \ldots \ldots . \ldots 4$

Proposição: Conjunto de índices associado a uma solução $\ldots \ldots \ldots \ldots \ldots \ldots \ldots \ldots 6$

Proposição: Número máximo de soluções - caso geral $\ldots \ldots \ldots \ldots \ldots \ldots \ldots \ldots \ldots 7$

Proposição: NP-completude da viabilidade do (GEiCP) $\ldots \ldots \ldots \ldots \ldots \ldots \ldots$ 


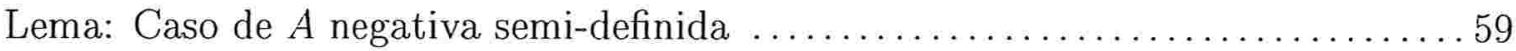

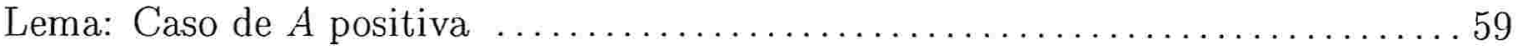

Lema: Caso de coluna com estrutura de sinal $\ldots \ldots \ldots \ldots \ldots \ldots \ldots \ldots \ldots \ldots \ldots \ldots \ldots \ldots \ldots \ldots$

Proposição: (EiCP) simétrico como problema de otimização $\ldots \ldots \ldots \ldots \ldots \ldots \ldots \ldots . \ldots \ldots$

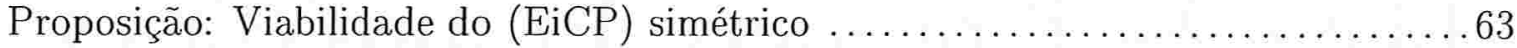

Proposição: Obtenção de ponto inicial conveniente - casos fáceis $\ldots \ldots \ldots \ldots \ldots 6$

Proposição: Obtenção de ponto inicial conveniente - caso geral $\ldots \ldots \ldots \ldots \ldots \ldots 67$

Proposição: Número máximo de soluções do (EiCP) simétrico $\ldots \ldots \ldots \ldots \ldots \ldots \ldots 7$

Proposição: (GEiCP) como problema de otimização $\ldots \ldots \ldots \ldots \ldots \ldots \ldots \ldots . \ldots \ldots$

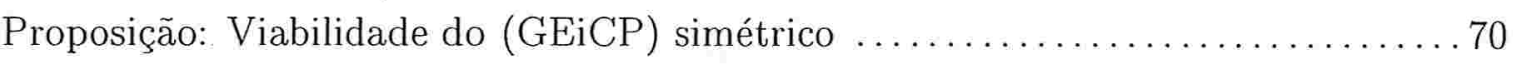

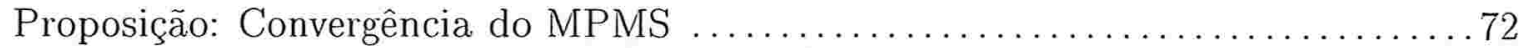

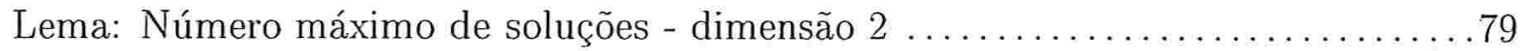

Lema: Viabilidade do (EiCP) com matriz P - dimensão $2 \ldots \ldots \ldots \ldots \ldots \ldots$

Lema: $(\mathrm{GEiCP})_{J}$ como sistema de polinômios em $x \ldots \ldots \ldots \ldots \ldots \ldots \ldots \ldots \ldots \ldots \ldots \ldots$

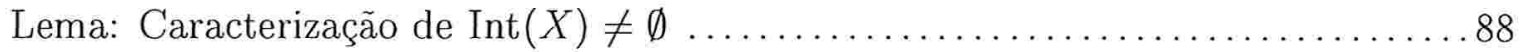

Lema: Comportamento assintótico de $f^{j}(\theta)=f\left(v^{0}+\theta\left(v^{i}-v^{0}\right)\right) \ldots \ldots \ldots \ldots .90$

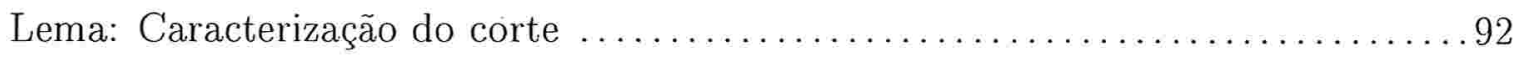

Lema: Comportamento de $f(\cdot)$ em direções paralelas ao corte $\ldots \ldots \ldots \ldots \ldots \ldots 93$

Proposição: Corretude do corte de concavidade $\ldots \ldots \ldots \ldots \ldots \ldots \ldots \ldots \ldots \ldots . \ldots \ldots$

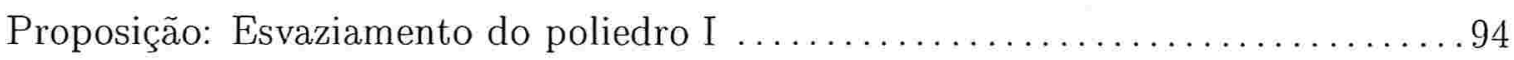

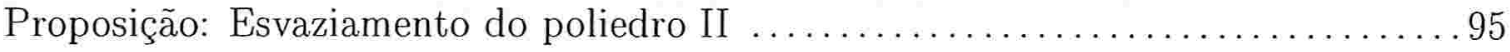

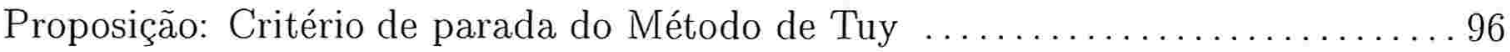

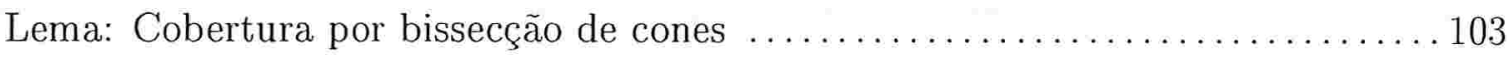

Lema: Cobertura por cones no Método de Thoai \& Tuy $\ldots \ldots \ldots \ldots \ldots \ldots \ldots \ldots$

Lema: Convergência de cones para subseqüências encaixadas ............... 106

Proposição: Convergência de $d_{k}$ no Método de Thoai \& Tuy $\ldots \ldots \ldots \ldots \ldots \ldots . \ldots 107$

Proposição: Convergência do Método de Thoai \& Tuy $\ldots \ldots \ldots \ldots \ldots \ldots \ldots \ldots$ 
Proposição: Equivalência de LCP e LCP-PPC $\ldots \ldots \ldots \ldots \ldots \ldots \ldots \ldots \ldots \ldots \ldots \ldots \ldots \ldots \ldots \ldots$

Lema: Classe de matrizes associadas a poliedros limitados $\ldots \ldots \ldots \ldots \ldots \ldots 112$

Lema: Corretude do corte de Tuy para o (LCP) $\ldots \ldots \ldots \ldots \ldots \ldots \ldots \ldots \ldots \ldots \ldots \ldots$

Proposição: Métodos de Tuy e Thoai \& Tuy com $f^{*}=0 \ldots \ldots \ldots \ldots \ldots \ldots \ldots \ldots \ldots \ldots \ldots$

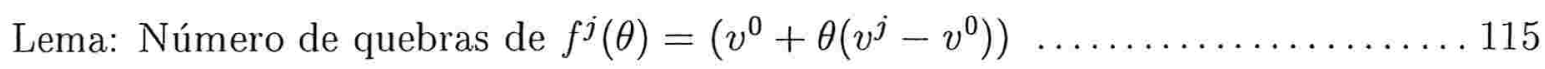

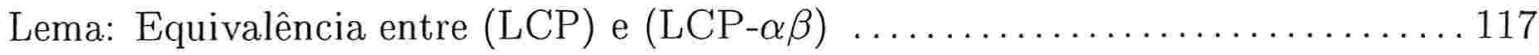

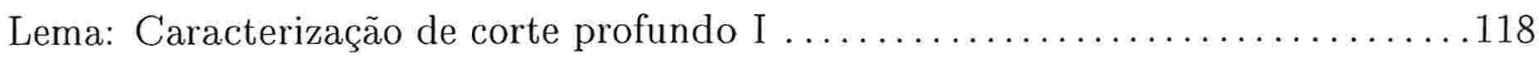

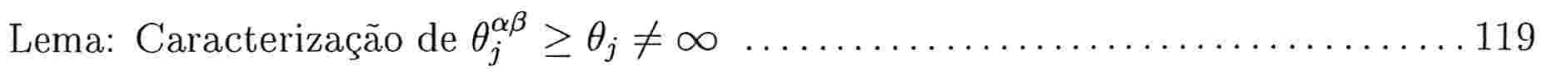

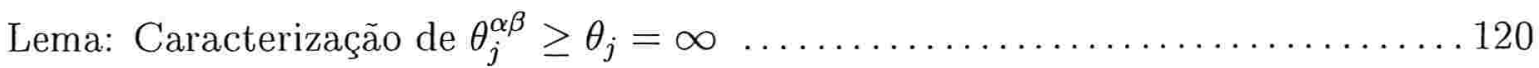

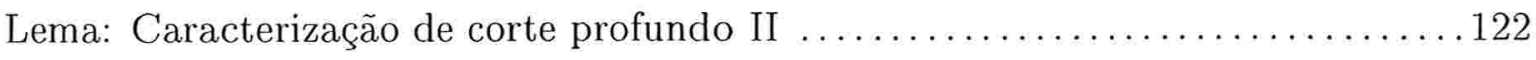

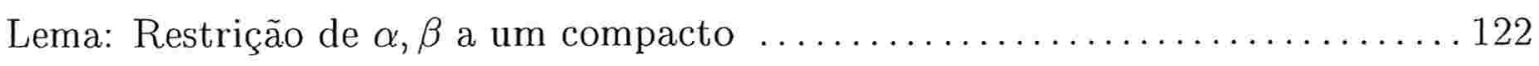

Proposição: Corretude do método de obtenção de corte profundo $\ldots \ldots \ldots \ldots \ldots \ldots$

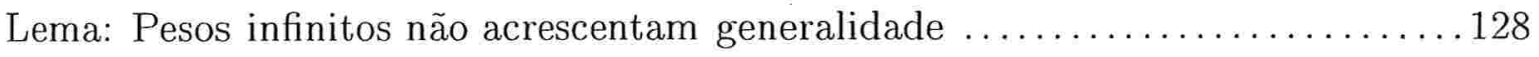




\section{Lista de Algoritmos e Heurísticas}

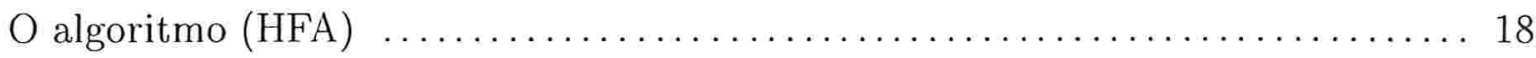

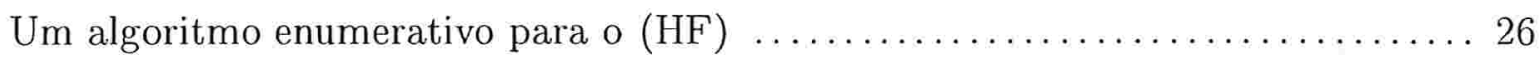

O Algoritmo SLCP para o Ajuste Linear de Dobradiças $\ldots \ldots \ldots \ldots \ldots \ldots \ldots \ldots . \ldots$

Conjunto Completo de Soluções do $(\mathrm{GEiCP})_{J} \ldots \ldots \ldots \ldots \ldots \ldots \ldots \ldots \ldots \ldots \ldots \ldots \ldots \ldots \ldots \ldots \ldots \ldots$

Método Projetado de Máxima Subida (MPMS)

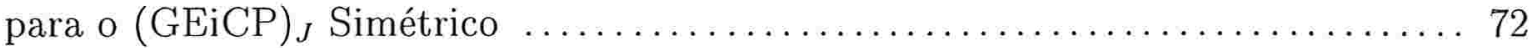

Método de Tuy (somente cortes de concavidade) $\ldots \ldots \ldots \ldots \ldots \ldots \ldots \ldots \ldots$

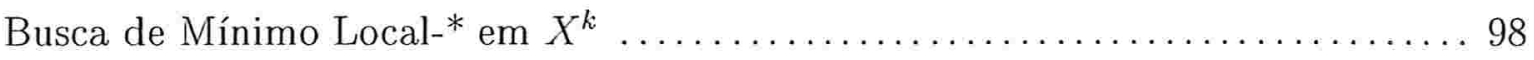

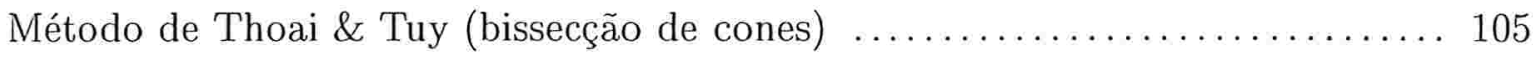

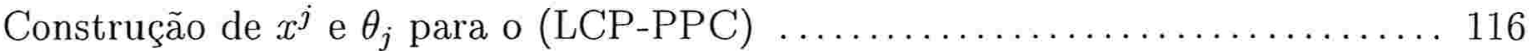

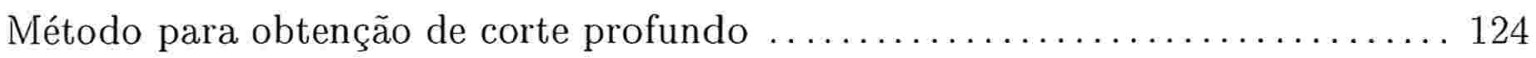

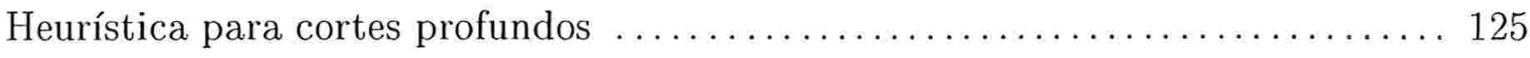

xix 


\section{Notações}

Notação 1 (Índices sobre-/sub-escritos)

Se $x \in \mathbb{R}^{n}$, denota-se suas coordenadas por $x_{1}, x_{2}, \ldots, x_{n}$. Todos os vetores são colunas.

Se $A \in M_{m \times n}(\mathbb{R})$ é uma matriz $m \times n$, denota-se por $A^{j}$ a j-ésima coluna de $A$, e por $A_{i}$ a i-ésima linha de $A$ :

$$
A=\left[\begin{array}{ccc}
\ddots & \vdots & \\
\cdots & a_{i j} & \cdots \\
& \vdots & \ddots
\end{array}\right]=\left[A^{1}\left|A^{2}\right| \cdots \mid A^{n}\right]=\left[\begin{array}{c}
\frac{A_{1}^{t}}{A_{2}^{t}} \\
\hline \vdots \\
\hline A_{m}^{t}
\end{array}\right] .
$$

Se I é um conjunto de índices, usam-se as notações $x_{I}=\left(x_{i}\right)_{i \in I}, A_{I}=\left(A_{i}\right)_{i \in I}$ e $A^{I}=\left(A^{i}\right)_{i \in I}$.

Notação 2 (Vetor unitário)

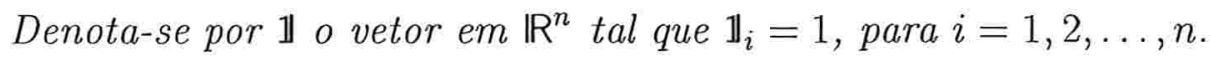

Notação 3 (Relações de ordem e ortantes não-negativo e positivo)

Sejam $x \in \mathbb{R}^{n}, y \in \mathbb{R}^{n}$, e seja $\geq_{\mathbf{R}}$ a relação de ordem usual em $\mathbb{R}$. Define-se:

$$
\begin{aligned}
& x \geq y \quad \Longleftrightarrow \quad x_{i} \geq_{\mathbf{R}} y_{i}, \text { para } i=1,2, \ldots, n ; \\
& x>y \Longleftrightarrow x_{i}>_{\mathbf{R}} y_{i}, \text { para } i=1,2, \ldots, n ;
\end{aligned}
$$


e denota-se por vecmax $\{x, y\}$ o vetor com componentes vecmax $\{x, y\}_{i}=\max \left\{x_{i}, y_{i}\right\}$.

Os ortantes não-negativo e positivo são denotados por

$$
\begin{aligned}
& \mathbb{R}_{+}^{n}=\left\{x \in \mathbb{R}^{n} \mid x \geq 0\right\} e \\
& \mathbb{R}_{++}^{n}=\left\{x \in \mathbb{R}^{n} \mid x>0\right\} .
\end{aligned}
$$

Notação 4 (Bola aberta e interior)

Denota-se a bola aberta de centro $\bar{x}$ e raio $\varepsilon$ por $B(\bar{x}, \varepsilon)=\left\{x \in \mathbb{R}^{n} \mid\|x-\bar{x}\|<\varepsilon\right\}$.

Se $A \subset \mathbb{R}^{n}$ denota-se o seu interior por $\operatorname{Int}(A)$ onde

$$
x \in \operatorname{Int}(A) \Longleftrightarrow \exists U \text { aberto t.q. } x \in U \subset A \text {. }
$$

Notação 5 (Soma de conjuntos)

Se $A, B \subset \mathbb{R}^{n}$ e $x \in \mathbb{R}^{n}$, denota-se por

$$
\begin{aligned}
& A+B=\{a+b \mid a \in A, b \in B\} e \\
& x+A=\{x+a \mid a \in A\} .
\end{aligned}
$$

\section{Notação 6 (Cone e casco convexo)}

Dados $n$ pontos $p_{1}, \ldots, p_{n}$ em $\mathbb{R}^{m}$, denota-se o cone e o casco convexo gerados por $p_{1}, \ldots, p_{n}$, respectivamente, por

$$
\begin{aligned}
& \operatorname{cone}\left\{p_{1}, \ldots, p_{n}\right\}=\left\{\sum_{i=1}^{n} \alpha_{i} p_{i} \mid \alpha \geq 0\right\} \\
& \operatorname{conv}\left\{p_{1}, \ldots, p_{n}\right\}=\left\{\sum_{i=1}^{n} \alpha_{i} p_{i} \mid \alpha \geq 0, \sum_{i=1}^{n} \alpha_{i}=1\right\} .
\end{aligned}
$$

Dada uma matriz $A$ e um conjunto de índices I, denota-se

$$
\begin{aligned}
& \operatorname{cone}\left(A_{I}\right)=\operatorname{cone}\left\{A_{i}\right\}_{i \in I}, \\
& \operatorname{conv}\left(A_{I}\right)=\operatorname{conv}\left\{A_{i}\right\}_{i \in I} .
\end{aligned}
$$




\section{Glossário}

Classes de Matrizes As seguintes classes de matrizes são importantes no estudo de problemas de complementaridade. Seja $M$ uma matriz real.

Matriz Adequada Para todo conjunto de índices $I$, se $\operatorname{det}\left(A_{I I}\right)=0$ então as linhas de $A_{I}$ são L.I., e as colunas de $A^{I}$ são L.I.

Matriz Copositiva Satisfaz $x^{t} M x \geq 0, \forall x \geq 0$.

Matriz Copositiva Estrita Satisfaz $x^{t} M x>0, \forall x \geq 0, x \neq 0$.

Matriz Diagonal Dominante Satisfaz, para todo $i$,

$$
\left|m_{i i}\right| \geq \sum_{j \neq i}\left|m_{i j}\right|
$$

Matriz $\mathrm{E}_{0}$ (Semi-Monótona) Satisfaz $\left.\begin{array}{l}z \geq 0 \\ z \neq 0\end{array}\right\} \Longrightarrow \exists k\left\{\begin{array}{r}z_{k}>0 \\ (M z)_{k} \geq 0 .\end{array}\right.$

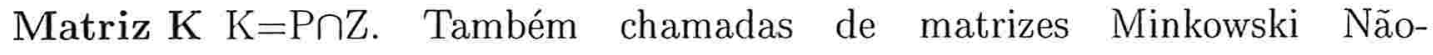
Singulares.

Matriz Não-Degenerada Tem todos os menores principais não-nulos.

Matriz $\mathbf{P}$ Tem todos os menores principais positivos.

Matriz $\mathbf{P}_{0}$ Tem todos os menores principais não-negativos.

Matriz Positiva Definida Satisfaz $x^{t} M x>0, \forall x \neq 0$. 
Matriz Positiva Semi-Definida Satisfaz $x^{t} M x \geq 0, \forall x$.

Matriz $R_{0}$ (Pseudo-Regular) Satisfaz

$$
\left.\begin{array}{rl}
z & \geq 0 \\
M z & \geq 0 \\
z^{t} M z & =0
\end{array}\right\} \Longrightarrow z=0 .
$$

Matriz Regular Satisfaz, para todo $\alpha \geq 0$,

$$
\left.\begin{array}{rl}
z & \geq 0 \\
M z+\alpha e & \geq 0 \\
t(M z+\alpha e) & =0
\end{array}\right\} \Longrightarrow z=0
$$

Matriz S (Stiemke) Satisfaz $\exists z \geq 0: M z>0$.

Matriz $\mathbf{S}_{0}$ Satisfaz $\exists z \geq 0, z \neq 0: M z \geq 0$.

Matriz Suficiente Satisfaz as condições $\left[z_{i}(M z)_{i} \leq 0, \forall i \Longrightarrow z^{t} M z=0\right]$ e $\left[z_{i}\left(M^{t} z\right)_{i} \leq 0, \forall i \Longrightarrow z^{t} M^{t} z=0\right]$.

Matriz Z Satisfaz $m_{i j} \leq 0, \forall i \neq j$.

LCP Problema de Complementaridade Linear (Linear Complementarity Problem).

MPEC Problemas de Programação Matemática com Restrições de Equilíbrio (Mathematical Programming with Equilibrium Constraints).

NCP Problema de Complementaridade Não-Linear (Nonlinear Complementarity Problem).

PL Programação Linear, ou também Problema (Programa) Linear.

PNL Programação Não-Linear (também Programa Não-Linear).

PQ Programação Quadrática (também Programa Quadrático). 


\section{Introdução}

Este trabalho aborda três problemas que têm em comum modelagens envolvendo condições de complementaridade, e propostas de solução com ferramentas de programação matemática.

O termo complementaridade, aqui utilizado, refere-se à condição

$$
x^{t} y=0
$$

onde $x, y \in \mathbb{R}_{+}^{n}$.

Condições de complementaridade surgem naturalmente no contexto de programação matemática. Considere, por exemplo, o problema de programação nãolinear

$$
\begin{cases}\min & f(x) \\ \text { s.a. } & g(x) \leq 0, i=1,2, \ldots, m\end{cases}
$$

onde $f: \mathbb{R}^{n} \rightarrow \mathbb{R}, g: \mathbb{R}^{n} \rightarrow \mathbb{R}^{m}$ são diferenciáveis, e $f(\cdot)$ e $g_{i}: \mathbb{R}^{n} \rightarrow \mathbb{R}$, $i=1,2, \ldots, m$, são convexas. Sob hipóteses de regularidade (por exemplo, a condição de qualificação $\exists x: g(x)<0$, de Slater), as condições de Kuhn-Tucker

$$
\left\{\begin{aligned}
-\nabla f(x) & =\mu^{t} \nabla g(x) \\
g(x) & \leq 0 \\
\mu & \geq 0 \\
\mu^{t} g(x) & =0
\end{aligned}\right.
$$


são condições necessárias e suficientes de otimalidade para o problema original. A condição de folgas complementares, $\mu^{t} g(x)=0$, é uma condição de complementaridade, que neste contexto garante que ou uma restrição $g_{i}(\cdot) \leq 0$ é a.tiva em $x$ (i.e., $g_{i}(x)=0$ ), ou o multiplicador de Lagrange $\mu_{i}$ associado é zero.

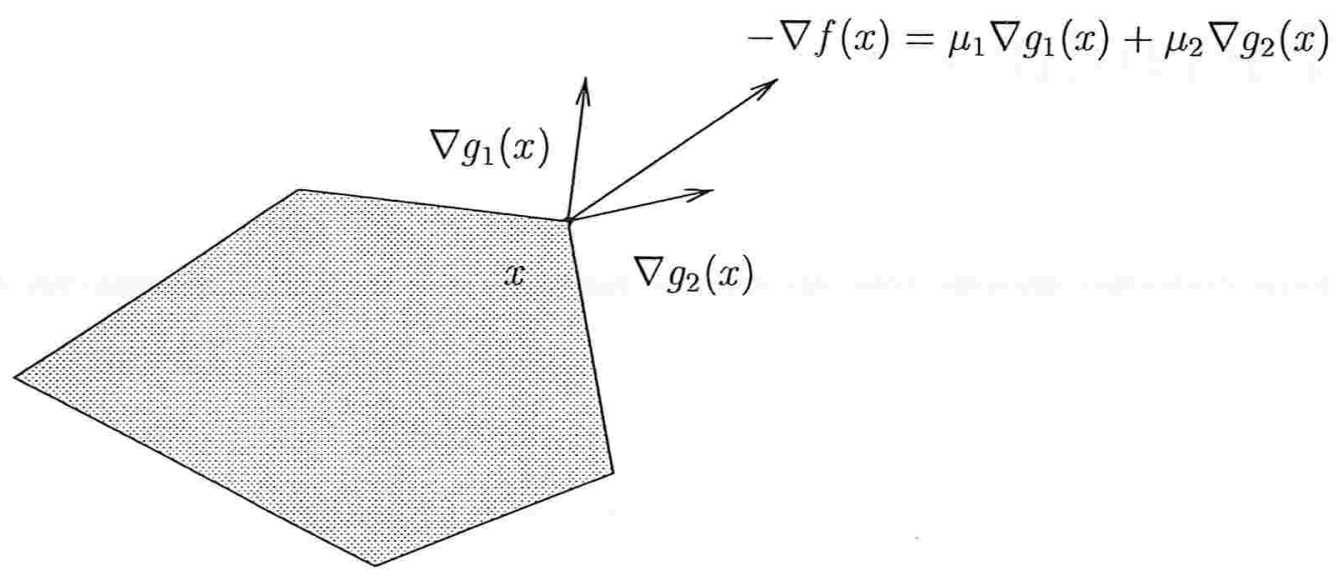

Em outras palavras, esta condição garante que apenas os gradientes das restrições ativas são utilizados na combinação linear para expressar o gradiente da função objetivo.

Problemas de complementaridade geralmente possuem um caráter combinatório, devido à natureza disjuntiva da condição de complementaridade (ou $x_{i}=0$, ou $\left.y_{i}=0\right)$. Um exemplo trivial disto é o sistema

$$
\left\{\begin{array}{l}
x+y=1 \\
x y=0 \\
x, y \in \mathbb{R}_{+}
\end{array}\right.
$$

que possui o conjunto solução $\{(1,0),(0,1)\} \in \mathbb{R}^{2}$. Condições de complementaridade são também utilizadas na modelagem de conjuntos não-convexos; como exemplo, a restrição $z \leq \max \{x,-2 x\}$ 
xxvii

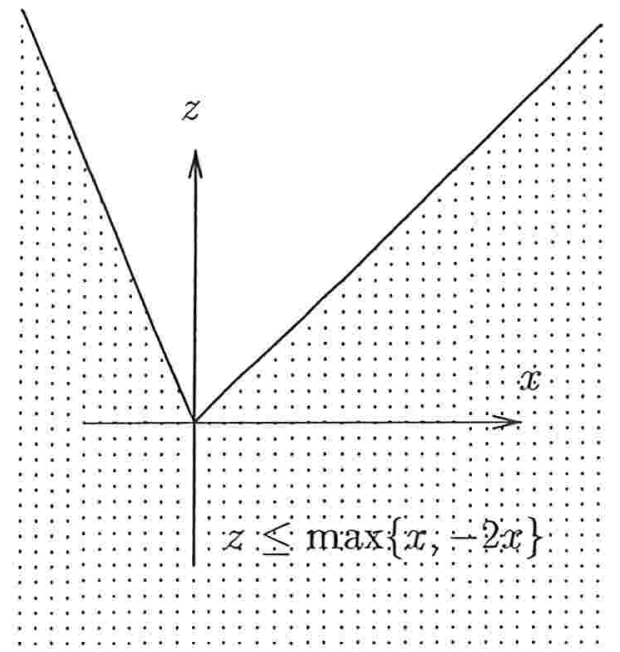

pode ser modelada como

$$
\left\{\begin{array}{l}
z \leq \sigma \\
\sigma \geq x \\
\sigma \geq-2 x \\
(\sigma-x)^{t}(\sigma+2 x)=0 .
\end{array}\right.
$$

De um modo geral, condições que representam situações de equilíbrio em matemática, como pontos de sela de Fritz John e Kuhn-Tucker, como o equilíbrio Walrasiano em economia matemática e o equilíbrio de Nash em teoria dos jogos, são representadas por condições de complementaridade [Harker \& Pang 1990, Cottle, Pang \& Stone 1992].

Os problemas abordados neste trabalho são instâncias de três classes importantes na área de complementaridade: Problemas de Complementaridade Linear (LCP - Linear Complementarity Problem) e Não-Linear (NCP - Nonlinear Complementarity Problem), e Problemas de Programação Matemática com Restrições de Equilíbrio (MPEC - Mathematical Programming with Equilibrium Constraints).

O Problema de Complementaridade Linear é: dados uma matriz $M \in \mathbb{R}^{n \times n}$ 
e um vetor $q \in \mathbb{R}^{n}$, encontrar $z \in \mathbb{R}^{n}$ tal que

$$
(L C P)\left\{\begin{aligned}
z & \geq 0 \\
w(z) & \geq 0 \\
z^{t} w(z) & =0
\end{aligned}\right.
$$

onde $w(z)=M z+q$. Este problema é equivalente ao problema quadrático

$$
\begin{cases}\min & f(z)=z^{t}(M z+q) \\ \text { s.a. } & M z+q \geq 0 \\ & z \geq 0\end{cases}
$$

no sentido que $z$ é solução ótima deste problema com $f(z)=0$ se, e somente se, $z$ é solução do (LCP); por outro lado o (LCP) é viável se, e somente se, o valor ótimo deste problema quadrático é 0. A literatura sobre o (LCP) é bastante extensa; alguns livros-texto e artigos de revisão são [Cottle, Pang \& Stone 1992, Murty 1988, Cottle 1976, Cottle \& Dantzig 1968, Eaves 1971, Júdice 1994, Júdice \& Mitra 1988]; outros trabalhos importantes sobre o (LCP) são [Al-Khayyal 1987, Bai \& Evans 1997, Cottle \& Dantzig 1970, Cottle \& Habetler 1970, Fukushima, Luo \& Pang 1998, Ha 1985, Júdice \& Faustino 1988a, Júdice \& Faustino 1988b, Júdice \& Faustino 1991, Júdice \& Faustino 1992, Júdice, Machado \& Faustino 1993, Júdice \& Pires 1992, Júdice \& Pires 1994, Mangasarian 1997, Pardalos \& Rosen 1988, Ramarao \& Shetty 1984, Sherali, Krishnamurty \& Al-Khayyal 1996, Sherali, Krishnamurty \& Al-Khayyal 1998, Thoai \& Tuy 1983, Ye \& Pardalos 1991].

Uma extensão natural do problema anterior é o Problema de Complementaridade Não-linear: dada uma função diferenciável $g: \mathbb{R}^{n} \rightarrow \mathbb{R}^{n}$, encontre $z \in \mathbb{R}^{n}$ tal que

$$
(N C P)\left\{\begin{aligned}
z & \geq 0 \\
g(z) & \geq 0 \\
z^{t} g(z) & =0 .
\end{aligned}\right.
$$


Este problema é uma especialização do problema de desigualdade variacional: dado $K \subseteq \mathbb{R}^{n}$ fechado e convexo, e $g: \mathbb{R}^{n} \rightarrow \mathbb{R}^{n}$, encontre $z \in K$ tal que

$$
(V I) \quad \forall k \in K,(k-z)^{t} g(z) \geq 0
$$

$\mathrm{O}$ (NCP) é obtido do (VI) com $K=\mathbb{R}_{+}^{n}$. Grande parte da literatura relevante para o problema de complementaridade não-linear está formulada no contexto do problema de desigualdade variacional. Os artigos [Harker \& Pang 1990, Cottle 1976, Karamardian 1969, Moré 1974, Peng 1995] fornecem um panorama da área; outros trabalhos importantes sobre o (NCP) são [Dirkse \& Ferris 1995, Karamardian 1971, Karamardian 1972, Ralph 1994, Robinson 1979, Robinson 1980, Robinson 1981, Robinson 1982].

Outra linha de generalização consiste em considerar as soluções dos problemas (LCP) e (NCP) como conjunto viável (geralmente não-convexo) de um problema de otimização. Os problemas de minimização sobre o (LCP) e o (NCP),

$$
\begin{aligned}
& (M L C P) \begin{cases}\min & c^{t} z \\
s . a . & z \geq 0 \\
& w(z)=M z+q \geq 0 \\
& z^{t} w(z)=0\end{cases} \\
& (M N C P) \begin{cases}\min & \varphi(z) \\
\text { s.a. } & z \geq 0 \\
& g(z) \geq 0 \\
& z^{t} g(z)=0\end{cases}
\end{aligned}
$$

são exemplos de problemas de Programação Matemática com Restrições de Equilíbrio. Alguns trabalhos sobre MPECs são [Luo, Pang \& Ralph 1996, Andreani \& Martinez 2001, Fukushima, Luo \& Pang 1998, Luo, Pang, Ralph \& Wu 1996, Mangasarian \& Pang 1997]. 
O capítulo 1 considera o problema de Ajuste de Dobradiças (HF - Hinge Fitting), proposto em [Breiman 1993, Pucar \& Sjöberg 1998]. Este problema consiste no ajustamento de uma função dobradiça, ou seja, uma função da forma $h(a)=\max \left\{x^{t} a, y^{t} a\right\}$, a $n$ pontos dados $\left(A_{i}, b_{i}\right) \in \mathbb{R}^{m}$. Através da modelagem da função máximo por meio de uma condição de complementaridade, este problema é visto como um MPEC com uma função objetivo convexa,

$$
(\mathrm{HF}) \begin{cases}\min & \|\sigma\| \\ \text { s.a. } & r=\sigma-A x+b \\ & s=\sigma-A y+b \\ & r, s \geq 0 \\ & r^{t} s=0,\end{cases}
$$

onde $\|\cdot\|$ é a norma utilizada para medição dos erros.

Para a norma Euclideana é proposto um método de enumeração implícita, que é polinomial no tamanho $n$ da amostra, para dimensão do espaço $m$ fixada. Verificase que os nós da árvore de enumeração estão associados a partições separáveis dos dados. Mostra-se que o tamanho da árvore de enumeração é uma função polinomial do tamanho da amostra, em dimensão fixada. Uma formulação alternativa para a norma $l_{1}$ como MPEC linear é também proposta, e para sua solução um método SLCP [Júdice \& Faustino 1992] é apresentado. Testes numéricos com os dois algoritmos estão incluídos, para avaliação da eficiência destes métodos.

Em seguida, no capítulo 2, é estudado o Problema de Valores Próprios Complementar (EiCP - Eigenvalue Complementarity Problem) com matrizes reais simétricas. Este problema surgiu no estudo de estados de equilíbrio estático de sistemas mecânicos com atrito unilateral [Júdice, Costa, Figueiredo \& Martins 2000], e é 
modelado como um problema de complementaridade não-linear (NCP) misto,

$$
\text { (EiCP): Encontre } \lambda>0, x \neq 0 \text { t.q. }\left\{\begin{array}{l}
w=(\lambda B-A) x \\
w \geq 0 \\
x \geq 0 \\
w^{t} x=0 .
\end{array}\right.
$$

Mostra-se que resolver o (EiCP) com $A$ e $B$ simétricas é equivalente a encontrar uma solução de equilíbrio do problema

$$
(O E i C P) \begin{cases}\max & \lambda(x) \\ \text { s.a. } & x \geq 0 \\ & e^{t} x=1,\end{cases}
$$

$\operatorname{com} \lambda(x)>0$. Uma condição necessária e suficiente de viabilidade é obtida que, quando verificada, fornece uma solução inicial $x^{0}$ tal que $\lambda\left(x^{0}\right)>0$, a partir da qual qualquer método local razoável de subida de gradiente converge para uma solução do (EiCP). Além disso, resultados semelhantes são obtidos para o Problema de Valores Próprios Complementar Geral (GEiCP - General EiCP) simétrico. Testes computacionais estão incluídos, mostrando que esta reformulação permite a resolução dos problemas simétricos em menor tempo e com maior robustez (certeza de resolução).

O último problema considerado é o Problema de Complementaridade Linear

$$
(L C P)\left\{\begin{aligned}
z & \geq 0 \\
w(z) & \geq 0 \\
z^{t} w(z) & =0
\end{aligned}\right.
$$

onde $X=\{z \mid z \geq 0$ e $w(z)=M z+q \geq 0\}$ é um conjunto compacto (capítulo 3). É apresentada uma técnica de aprofundamento de cortes de concavidade para o (LCP), baseada na reformulação do mesmo como uma família de Problemas de Programação 
Côncava, parametrizada em $\alpha, \beta \in \mathbb{R}_{+}^{n}$,

$$
(L C P-\alpha \beta) \begin{cases}\min & f^{\alpha \beta}(z)=\sum_{i=1}^{n} \min \left\{\alpha_{i} z_{i}, \beta_{i} w_{i}(z)\right\} \\ & z \in X .\end{cases}
$$

Esta técnica visa aumentar o volume da região cortada, reajustando os parâmetros que definem a função objetivo côncava a cada passo, e obtém cortes mais profundos do que o corte de Tuy sempre que possível, isto é, sempre que o corte de Tuy não é eficiente em relação à família de problemas considerada. São analisados os métodos de [Tuy 1964] e [Thoai \& Tuy 1983], baseados no uso de cortes de concavidade, e o impacto da técnica de aprofundamento nos mesmos. Detalhes de implementação são discutidos, e alguns testes computacionais são apresentados, a fim de revelar potencialidades e fraquezas de cada um dos métodos. Em geral, constata-se que estes métodos de solução do (LCP) não têm bom desempenho computacional, e não são adequados para problemas de médio e grande porte.

Possíveis extensões de pesquisa são relacionados ao final de cada capítulo, juntamente com uma breve conclusão dos resultados do respectivo problema. Em vista disso, a forma escolhida para conclusão geral (capítulo 4) é a de uma exposição das principais contribuições deste trabalho aos problemas considerados. 


\section{Capítulo 1}

\section{O Problema de Ajuste de}

\section{Dobradiças}

Problemas de ajuste de curvas por mínimos quadrados aparecem freqüentemente na literatura, e têm importantes aplicações em métodos estatísticos, em Sistemas Dinâmicos e em Processamento de Sinais, entre outros. Problemas de ajustamento (fitting) de funções lineares e não-lineares podem ser resolvidos por uma variedade de algoritmos, com métodos de solução que vão desde Otimização Discreta e Contínua, até Redes Neurais, Ondaletas (Wavelets) e Geometria Computacional [Bertsekas \& Tsitsiklis 1996, Cottle 1976, Hassoun 1995].

O problema de ajustamento de funções não-lineares pressupõe a escolha de uma função para medição dos erros, bem como a escolha de funções básicas. A norma Euclideana das diferenças entre a função original e a aproximação desta nos pontos dados é a forma mais comum de medição dos erros; as normas $l_{1}$ e $l_{\infty}$ são possíveis alternativas que definem diferentes objetivos de aproximação. A escolha das funções básicas depende da classe de funções a serem aproximadas, e podem variar desde funções indicadoras, polinômios e "splines" até funções trigonométricas e sigmóides. 
O modelo de hiperplanos dobrados (HH - Hinging Hyperplanes) recebeu atenção na literatura recente [Breiman 1993, Pucar \& Sjöberg 1998] como uma alternativa às funções sigmóides dos modelos de redes neurais para ajustamento de funções não-lineares. O modelo HH usa funções dobradiças, ou seja, funções da for$\operatorname{ma} h(a)=\max \left\{x^{t} a+x_{m}, y^{t} a+y_{m}\right\}$ ou $h(a)=\min \left\{x^{t} a+x_{m}, y^{t} a+y_{m}\right\}$, como funções básicas em expansões tais como

$$
f^{k}(a)=\sum_{i=1}^{k} h_{i}(a),
$$

onde $f^{k}(\cdot)$ é uma aproximação de uma função $f(\cdot)$, cuja fórmula é desconhecida. Se a função $f(\cdot)$ for suficientemente suave, então [Breiman 1993] existe um $\alpha>0$ tal que para qualquer $k \in \mathbb{N}$, existem $k$ funções dobradiças $h_{1}, \ldots, h_{k}$, satisfazendo

$$
\left\|f-\sum_{i=1}^{k} h_{k}\right\|^{2} \leq \frac{\alpha}{k} .
$$

A aproximação de funções não-lineares por somas de funções dobradiças, como acima, é um caso especial da aproximação por funções lineares por trechos. A expressão $\sum_{i=1}^{k} h_{k}$ define uma função linear por trechos com a propriedade que o seu conjunto de descontinuidades é uma união de variedades afins.

Um esquema de aproximação baseado no modelo HH foi proposto em [Breiman 1993], e consiste em ajustar aos valores da função $f$ dados uma dobradiça $h_{1}$, depois reajustar a diferença $f-h_{1}$ a uma dobradiça $h_{2}$, e então reajustar a diferença $f-h_{1}-h_{2}$ a uma dobradiça $h_{3}$, e assim por diante. Quando $k$ funções dobradiças forem computadas, pode-se redefinir cada uma das $h_{j}$ 's, em ordem, como a solução do problema de ajuste de dobradiças aos valores de $\left(f-\sum_{i \neq j} h_{i}\right)$, e continuar refinando a aproximação até que não se obtenha qualquer melhoria significativa.

Ainda em [Breiman 1993], um algoritmo iterativo para o problema de ajustamento de funções dobradiças foi proposto, chamado Algoritmo para Obtenção de Dobradiça (HFA - Hinge Finding Algorithm). Este é um método de ponto-fixo que 
particiona os dados em dois conjuntos, e resolve independentemente um problema de regressão linear para cada conjunto. As duas funções lineares obtidas são usadas para definir uma nova partição dos dados originais, que é usada como entrada para a próxima iteração. Diz-se que o algoritmo converge se as partições em duas iterações seguidas são idênticas. Quando o algoritmo converge, a solução obtida é um minimizador local do problema de mínimos quadrados. Mais ainda, se os dados originais representam medidas exatas de uma função dobradiça, e a partição original não está muito distante da correta, então o algoritmo converge [Breiman 1993]. Apesar de sua simplicidade e fácil implementação, esta abordagem tem desvantagens severas:

1. Se, em uma iteração, é obtida uma partição trivial, isto é, uma partição com o conjunto vazio de um lado, o algoritmo converge prematuramente. A última solução corresponde à solução da regressão linear para os dados originais.

2. O algoritmo pode ciclar entre um certo número de dobradiças. Neste caso o algoritmo não converge e não há garantia de otimalidade local.

Em [Pucar \& Sjöberg 1998] é proposta uma modificação do método a fim de garantir convergência global para uma solução localmente ótima. O HFA é visto como um método de Newton, e uma busca linear é introduzida para garantir decréscimo da função erro a cada iteração. A modificação proposta corresponde a um amortecimento do método de Newton.

Como acontece freqüentemente ao aplicar-se métodos de otimização local para problemas não-convexos, a qualidade dos mínimos locais obtidos depende fortemente da solução inicial. Para fins práticos, recomenda-se sempre a repetição do método a partir de várias soluções iniciais distintas.

A grande variação observada na qualidade dos mínimos locais em experiências práticas leva à consideração de um método de otimização global para o problema de 
ajuste de funções dobradiças. O presente trabalho trata deste tópico ao explorar duas reformulações do problema como MPECs. O problema de ajuste de dobradiças com erro dado pela norma Euclideana (Ajuste Euclideano de Dobradiças) é formulado como um MPEC quadrático, e o ajuste de dobradiças com erro dado pela norma $l_{1}$ (Ajuste Linear de Dobradiças) é formulado como um MPEC linear.

Um método de enumeração implícita para a solução global do MPEC quadrático é proposto, método este polinomial no tamanho $n$ da amostra, para dimensão $m$ do espaço fixada. Mostra-se que os cones complementares enumerados pelo método estão sempre associados a partições separáveis dos dados (seção 1.1), e com isso provase que o tamanho da árvore de enumeração é uma função polinomial do tamanho da amostra, em dimensão fixada. Alguns experimentos computacionais com este algoritmo enumerativo foram realizados, para problemas de médio porte. Os resultados aqui relatados mostram que a qualidade dos ajustamentos é melhorada substancialmente em relação àqueles obtidos pelo algoritmo HFA amortecido.

Para o problema de Ajuste Linear de Dobradiças, é apresentado um algoritmo baseado na solução de uma seqüência de LCPs gerais (SLCP) [Júdice \& Faustino 1992], e investiga-se o desempenho deste algoritmo. A experiência computacional apresentada mostra que este método processa eficientemente os mesmos problemas de médio porte reformulados com a norma $l_{1}$.

A estrutura deste capítulo é a seguinte. A formulação e propriedades gerais do problema de Ajuste Euclideano de Dobradiças são apresentadas na seção 1.1. Os algoritmos HFA e HFA amortecido são revisados na seção 1.2. O algoritmo para solução global do problema é discutido na seção 1.3. Na seção 1.4 é apresentado o problema de Ajuste Linear de Dobradiças, bem como o método de solução baseado no SLCP. A experiência computacional é relatada na seção 1.5 e algumas conclusões são apresentadas na seção 1.6. 


\subsection{O Problema do Ajuste de Funções Dobradiças}

Sejam $\left(a_{i}, b_{i}\right) \in \mathbb{R}^{m-1} \times \mathbb{R}=\mathbb{R}^{m}$, para $i=1, \ldots, n$, as medidas dadas de uma função $b=f(a)$. O objetivo é aproximar $f(\cdot)$ por uma função dobradiça $h(a)=\max \left\{a^{t} x+x_{m}, a^{t} y+y_{m}\right\}$ minimizando o quadrado da norma Euclideana dos erros da aproximação. Para simplificar a notação, os pontos dados são escritos como $A_{i}=\left(a_{i}^{t}, 1\right) \in \mathbb{R}^{m}$ e correspondentemente $x=\left(\bar{x}, x_{m}\right) \in \mathbb{R}^{m}$, de modo que $A_{i} x=a_{i}^{t} \bar{x}+x_{m}$. O problema é

$$
\min _{x, y} \sum_{i=1}^{n}\left(\max \left\{A_{i} x, A_{i} y\right\}-b_{i}\right)^{2} .
$$

Modelando os erros da somatória acima como uma variável $\sigma$ o problema torna-se

$$
\left\{\begin{array}{ll}
\min & \sum_{i=1}^{n} \sigma_{i}^{2} \\
\text { s.a. } & \sigma_{i} \geq A_{i} x-b_{i} \\
& \sigma_{i} \geq A_{i} y-b_{i} \\
& \left(\sigma_{i}-A_{i} x+b_{i}\right)\left(\sigma_{i}-A_{i} y+b_{i}\right)=0,
\end{array} \quad i=1, \ldots, n\right.
$$

que é um MPEC com função objetivo quadrática. Para futura referência este problema é escrito abaixo em notação matricial, e é chamado de problema de Ajuste (Euclideano) de Dobradiças (HF - Hinge Fitting).

Definição 1.1 (Problema de Ajuste Euclideano de Dobradiças - HF)

$$
(H F) \begin{cases}\min & \sigma^{t} \sigma \\ \text { s.a. } & r=\sigma-A x+b \\ & s=\sigma-A y+b \\ & r, s \geq 0 \\ & r^{t} s=0\end{cases}
$$




\section{Soluçōes Viáveis e Partiçōes Separáveis}

É evidente, por construção, que a identidade $\sigma=\operatorname{vecmax}\{A x, A y\}-b$ é válida para qualquer solução viável de (HF), onde vecmax é a função máximo componente a componente. Isto permite que uma solução seja identificada simplesmente como $(x, y)$, visto que os valores $\sigma, r$ e $s$ são determinados univocamente. Qualquer solução $(x, y)$ do problema acima define uma função dobradiça

$$
h(a)=\max \left\{a^{t} x, a^{t} y\right\}
$$

assim como induz uma separação dos dados originais em três grupos, de acordo com as relações $A_{k} x<A_{k} y, A_{k} x=A_{k} y$ e $A_{k} x>A_{k} y$. Esta separação torna-se uma partição através de uma convenção sobre os índices $k$ tais que $A_{k} x=A_{k} y$. Assim, por convenção, diz-se que a solução $(x, y)$ induz uma partição dos dados nos conjuntos de índices

$$
\begin{aligned}
& P=\left\{k \mid A_{k} x \geq A_{k} y\right\} \\
& Q=\left\{k \mid A_{k} x<A_{k} y\right\} .
\end{aligned}
$$

A figura abaixo mostra a função dobradiça $h(a)$ definida por uma solução $(x, y)$, bem como a partição do conjunto de índices $\{1,2, \ldots, n\}$ induzida. Para simplificar, uma tal partição do conjunto de índices dos dados será também chamada de partição dos dados.

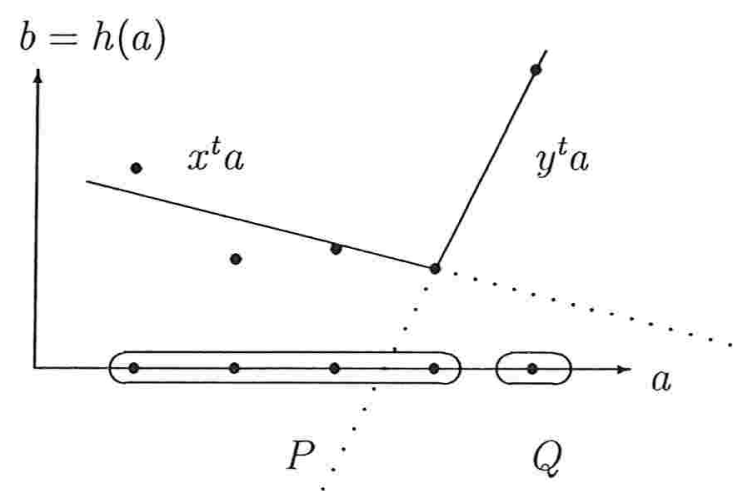


Note que a presença de um ponto na dobra da função dobradiça, isto é, na interseç̧ão dos hiperplanos definidos por $x^{t} a$ e $y^{t} a$, é absolutamente inofensiva, e a escolha de deixar este ponto no conjunto $P$ ou $Q$ é puramente convencional. Note ainda que alguma convenção torna-se necessária para que se possa associar qualquer função dobradiça a uma partição dos dados.

Definição 1.2 (Partição Separável)

Uma partição $(P, Q)$ de $\{1,2, \ldots, n\}$ é chamada de partição separável (de $\{1,2, \ldots, n\})$ se existe um hiperplano $\left\{a \mid a^{t} z=0\right\}$ tal que

$$
\begin{aligned}
& P=\left\{k \mid A_{k} z \geq 0\right\} \\
& Q=\left\{k \mid A_{k} z<0\right\} .
\end{aligned}
$$

No último exemplo a partição $(P, Q)$ é definida pelo hiperplano $\left\{a \mid a^{t}(x-y)=0\right\}$. Por convenção, os pontos $A_{k}$ incidentes sobre o hiperplano (i.e., tais que $A_{k} z=0$ ) pertencem ao conjunto $P$.

É fácil ver (lema 1.3 a seguir) que partições separáveis correspondem a conjuntos convexos estritamente separáveis, considerando os cascos convexos $\operatorname{conv}\left\{A_{k}\right\}_{k \in P}$ e $\operatorname{conv}\left\{A_{k}\right\}_{k \in Q}$ dos dados particionados. Isto explica que as partições $(P, Q)$ e $(Q, P)$ sejam consideradas equivalentes para os fins deste trabalho.

\section{Lema 1.3 (Partiçōes separáveis sāo estritamente separáveis)}

$(P, Q)$ é uma partição separável se, e somente se, $(Q, P)$ é uma partição separável; em particular, existe um z tal que

$$
\begin{aligned}
& P=\left\{k \mid A_{k} z>0\right\} \\
& Q=\left\{k \mid A_{k} z<0\right\} .
\end{aligned}
$$


Prova : Seja $z=\left(\bar{z}, z_{m}\right)$ o hiperplano da definição de separabilidade de $(P, Q)$. Tome $\bar{\gamma}=\frac{1}{2} \max \left\{A_{k} z \mid k \in Q\right\}<0$, e considere $z^{\prime}=\left(\bar{z}, z_{m}-\bar{\gamma}\right)$.

Lembrando que $A_{k m}=1$, segue que $A_{k} z^{\prime}=A_{k} z-\bar{\gamma}$, e portanto

$$
\begin{aligned}
& P=\left\{k \mid A_{k} z^{\prime}>0\right\} \\
& Q=\left\{k \mid A_{k} z^{\prime}<0\right\} .
\end{aligned}
$$

Com isto $z^{\prime}$ define a partição $(P, Q)$ e $-z^{\prime}$ define a partição $(Q, P)$.

O conceito de partições separáveis aparece explicitamente em [Cover 1965] e [Harding 1967], com o nome de "partições induzidas por hiperplanos", e é estreitamente relacionado ao conceito de "partições do espaço induzida por hiperplanos" [Schläfli 1852]. Esta relação é explicada sucintamente na observação 1.7 (seção 1.3). Uma abordagem recente sobre partições separáveis generalizadas pode ser encontrada em [Alon \& Onn 1999], por exemplo.

Dada uma partição $(P, Q)$, o problema

$$
\left(\mathrm{HF}_{P Q}\right) \begin{cases}\min & \sigma^{t} \sigma \\ \text { s.a. } & r=\sigma-A x+b \\ & s=\sigma-A y+b \\ & r_{P}=0 \\ & s_{Q}=0 \\ & r, s \geq 0\end{cases}
$$

é intimamente relacionado a (HF). Note que para toda solução viável de $\left(\mathrm{HF}_{P Q}\right)$ a identidade $\sigma=\operatorname{vecmax}\{A x, A y\}-b$ permanece válida, mas também valem $\sigma_{P}=A_{P} x-b_{P}\left(\right.$ pois $\left.r_{P}=0\right)$ e $\sigma_{Q}=A_{Q} y-b_{Q}$ (pois $\left.s_{Q}=0\right)$. Note ainda. que qualquer solução viável de $(\mathrm{HF})$ é também uma solução viável de ( $\left.\mathrm{HF}_{P Q}\right)$ para $P=\left\{k \mid A_{k} x \geq A_{k} y\right\}$ e $Q=\left\{k \mid A_{k} x<A_{k} y\right\}$. Isso explica que apenas partições separáveis mereçam consideração do ponto de vista pragmático, e somente estas são 
consideradas pelas abordagens algorítmicas aqui apresentadas. No entanto, uma breve análise das partições não-separáveis é apresentada ao final desta seção.

As restrições $r_{P}=0$ e $s_{Q}=0$, juntamente com $r, s \geq 0$, definem um cone em $\mathbb{R}_{+}^{n} \times \mathbb{R}_{+}^{n}$ que é chamado de cone de complementaridade, pois a condição de complementaridade $r^{t} s=0$ é satisfeita automaticamente.

\section{Lema 1.4 (Partiçōes separáveis e cones de complementaridade)}

Se $(P, Q)$ é uma partição separável, então o cone de complementaridade definido por $r_{P}=0, s_{Q}=0$, e $r, s \geq 0$, está associado ao fecho das soluções $(x, y)$ que definem a mesma partição separável $(P, Q)$.

Prova : Isto é conseqüência direta do fato que, sob complementaridade $\left(r^{t} s=0\right)$, valem as equivalências:

$$
\begin{aligned}
& r_{P}=0 \Longleftrightarrow s_{P}-r_{P} \geq 0 \Longleftrightarrow A_{P} x \geq A_{P} y \\
& s_{Q}=0 \Longleftrightarrow r_{Q}-s_{Q} \geq 0 \Longleftrightarrow A_{Q} x \leq A_{Q} y .
\end{aligned}
$$

Assim, considere uma solução $(x, y)$ viável em (HF) tal que $r_{P}=0 \mathrm{e}$ $s_{Q}=0$. Pelo lema 1.3, existe um vetor $z$ tal que

$$
\begin{aligned}
& P=\left\{k \mid A_{k} z>0\right\} \\
& Q=\left\{k \mid A_{k} z<0\right\} .
\end{aligned}
$$

Seja $\bar{\gamma}=\frac{1}{2} \max \left\{A_{k} z \mid k \in Q\right\}<0$, e note que $A_{Q} z<\bar{\gamma} 1$. Para cada $\varepsilon>0$, a solução $\left(x^{\varepsilon}, y\right)=(x+\varepsilon z, y)$ é viável em (HF) e define a mesma partição $(P, Q)$, pois

$$
\begin{aligned}
& A_{P}(x+\varepsilon z)=A_{P} x+\varepsilon A_{P} z>A_{P} y, \mathrm{e} \\
& A_{Q}(x+\varepsilon z)=A_{Q} x+\varepsilon A_{Q} z<A_{Q} y+\varepsilon \bar{\gamma} \rrbracket<A_{Q} y .
\end{aligned}
$$

Assim, definindo-se $\sigma^{\varepsilon}=\operatorname{vecmax}\{A(x+\varepsilon z), A y\}-b$ tem-se, pelas desigualdades acima, que $r_{P}^{\varepsilon}=0$ e $s_{Q}^{\varepsilon}=0$. Portanto, $\left(x^{\varepsilon}, y\right)$ é viável em 
$\left(\mathrm{HF}_{P Q}\right), \mathrm{e}$

$$
\lim _{\varepsilon \longrightarrow 0} x^{\varepsilon}=x
$$

o que mostra que $(x, y)$ está no fecho das soluções que definem a partição $(P, Q)$.

Observe que uma solução $(x, y)$ viável em $\left(\mathrm{HF}_{P Q}\right)$ poderá definir uma partição $\left(P^{\prime}, Q^{\prime}\right)$ diferente de $(P, Q)$, pois a restrição $s_{Q}=0$ não garante a desigualdade estrita $A_{Q} x<A_{Q} y$. Isso é conseqüência do fato que os subproblemas são definidos por conjuntos fechados. No entanto, sempre valem as identidades

$$
\begin{aligned}
& P^{\prime}=\left\{k \mid A_{k} x \geq A_{k} y\right\}=P \cup\left\{k \mid A_{k} x=A_{k} y\right\} \\
& Q^{\prime}=\left\{k \mid A_{k} x<A_{k} y\right\}=Q \backslash\left\{k \mid A_{k} x=A_{k} y\right\} .
\end{aligned}
$$

Estas identidades fornecem uma caracterização do conjunto de soluções de $\left(\mathrm{HF}_{P Q}\right)$ em relação às partições separáveis associadas.

\section{Lema 1.5 (Existência de solução ótima)}

O problema (HF) possui solução ótima. Sendo $\bar{b}=\frac{1}{n} \sum_{i=1}^{n} b_{i}$, tem-se

$$
0 \leq \text { Valor Ótimo }(H F) \leq \sum_{i=1}^{n}\left(b_{i}-\bar{b}\right)^{2}
$$

Prova : A existência de solução ótima para os subproblemas $\left(\mathrm{HF}_{P Q}\right)$ para qualquer partição separável $(P, Q)$ está garantida pois cada um destes problemas é um problema de programação quadrática convexo com uma função objetivo não-negativa (Teorema de Frank-Wolfe, vide por exemplo [Cottle, Pang \& Stone 1992], teorema 2.8.1). Assim, o problema original (HF) possui uma solução ótima, que é uma dentre as soluções ótimas dos problemas $\left(\mathrm{HF}_{P Q}\right)$.

A limitação do valor ótimo de (HF) segue do fato que a função objetivo é limitada inferiormente por 0 , e o limitante superior acima é dado pela 
solução viável $r=s=0, x=y=(0, \ldots, 0, \bar{b})^{t}$ e $\sigma=(\bar{b}, \ldots, \bar{b})^{t}-b$, onde $\bar{b}=\frac{1}{n} \sum_{i=1}^{n} b_{i}$.

Na realidade, a solução viável indicada acima também é viável para qualquer $\left(\mathrm{HF}_{P Q}\right)$, e portanto os valores ótimos destes subproblemas também estão nesta faixa de valores.

\section{O Número de Partições Separáveis}

As considerações acima motivam a seguinte questão: quantas partições separáveis de $n$ pontos em $\mathbb{R}^{m}$ existem? A resposta de pior caso é $2^{n-1}$, já que as partições $(P, Q)$ e $(Q, P)$ são equivalentes. Este limite superior é atingido por exemplo quando $n=m$ e os pontos escolhidos são $e^{i}=(0, \ldots, 1,0, \ldots, 0)^{t}$. Deve-se notar, entretanto, que este é um caso de solução trivial do problema original, pois dados $m$ pontos onde $f: \mathbb{R}^{m-1} \longrightarrow \mathbb{R}$ é calculada, existe um hiperplano que aproxima $f(\cdot)$ exatamente nestes $m$ pontos, fornecendo erro total nulo. A independência linear dos pontos corresponde à unicidade do hiperplano do exemplo.

A seguir mostra-se que, para dimensão $m$ fixada, o número de partições separáveis é limitado polinomialmente como função de $n$. Este é um resultado clássico, refraseado no presente contexto.

Teorema 1.6 (Schläfli 1852)

O número máximo de partições separáveis de $n$ pontos em $\mathbb{R}^{m}$ é

$$
v_{m}(n)=\sum_{i=0}^{m}\left(\begin{array}{c}
n-1 \\
i
\end{array}\right)
$$

este limite superior é atingido quando os pontos dados estão em posição geral (i.e. não existem $m+1$ pontos na mesma variedade linear $(m-1)$-dimensional). 


\section{Observação 1.7 (Partições do espaço $\times$ partições de pontos)}

O teorema original [Schläfli 1852] considera o número de partições do espaço por $n$ hiperplanos em posição geral, e prova o resultado com igualdade. Isto é equivalente a considerar partições separáveis de $n$ pontos em posição geral. Note que se os $n$ hiperplanos são dados por

$$
\begin{gathered}
A_{1} z=0 \\
A_{2} z=0 \\
\vdots \\
A_{n} z=0
\end{gathered}
$$

(recorde que $A_{k}=\left(a_{k}, 1\right)$ define simultaneamente o vetor normal e a translação do hiperplano), então uma "partição do espaço" corresponde ao conjunto dos pontos z que satisfazem

$$
\begin{gathered}
A_{1} z \geq 0 \\
A_{2} z \leq 0 \\
\vdots \\
A_{n} z \geq 0
\end{gathered}
$$

para uma particular substituição das igualdades anteriores por desigualdades. Definindo

$$
\begin{aligned}
& P=\{k \mid \quad "=" \text { foi substituído por " } \geq "\} \\
& Q=\{k \mid \quad "=" \text { foi substituído por " } \leq "\}
\end{aligned}
$$

é possível passar de uma interpretação para outra, trocando o papel de pontos e hiperplanos.

Prova : A prova é por indução em $(m, n) \in \mathbb{N}^{2}$.

Os casos de base da indução são os pares $(m, n)$ tais que $n \leq m$. Quando $n \leq m$, o número máximo de partições possíveis é $2^{n-1}$. Mais ainda, sempre que os pontos estão em posição geral (neste caso, quando são 
linearmente independentes), qualquer partição pode ser separada por um hiperplano, e assim $v_{m}(n)=2^{n-1}$.

Suponha, por indução, que o teorema vale para todo $m \leq \bar{m}$ e para todo $n \leq \bar{n}$. Mostra-se que o resultado vale para $m=\bar{m}$ e $n=\bar{n}+1$, e isto juntamente com as bases de indução estabelece o resultado para $(m, n) \in \mathbb{N}^{2}$.

Seja $m=\bar{m}$ e $n=\bar{n}+1$, e considere os pontos dados $p_{1}, \ldots, p_{n}$. Sem perda de generalidade, considere que existe um hiperplano $\mathcal{H}$ separando $p_{1}$ de $p_{2}, \ldots, p_{n}$ (i.e., considere que $p_{1}$ é um vértice de $\operatorname{conv}\left\{p_{1}, \ldots, p_{n}\right\}$ ). Considere $p_{2}^{\prime}, \ldots, p_{n}^{\prime}$ as projeções dos pontos originais em $\mathcal{H}$, na direção de $p_{1}$ (cada $p_{k}^{\prime}$ está no segmento de reta $\left[p_{1}, p_{k}\right]$ ), como na figura abaixo.

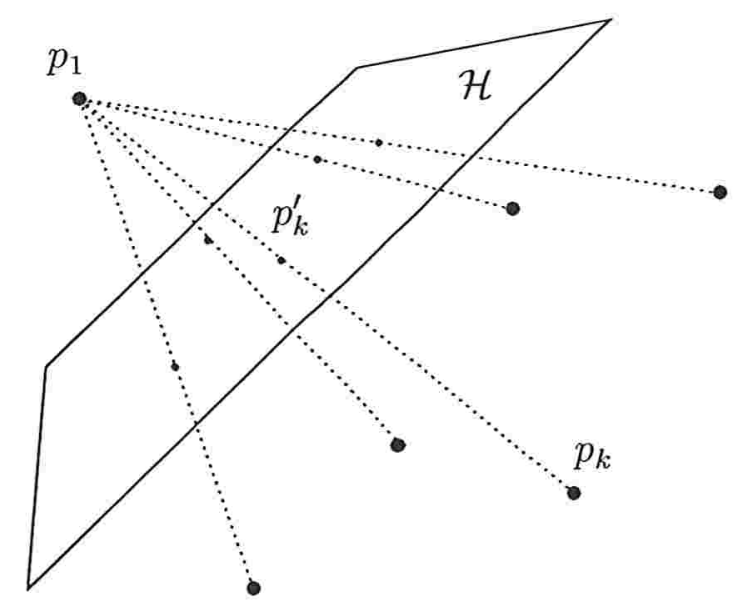

Note que toda partição separável $(P, Q)$ dos pontos originais induz uma partição separável $\left(P^{\prime}, Q^{\prime}\right)$ dos pontos $p_{2}, \ldots, p_{n}$, fazendo $P^{\prime}=P \backslash\{1\}$ e $Q^{\prime}=Q \backslash\{1\}$. Sempre que duas partições induzidas $\left(P^{\prime}, Q^{\prime}\right)$ e $\left(I^{\prime}, J^{\prime}\right)$ são idênticas, então os mesmos conjuntos de índices $\left(P^{\prime}, Q^{\prime}\right)=\left(I^{\prime}, J^{\prime}\right)$ definem uma partição separável dos pontos projetados $p_{2}^{\prime}, \ldots, p_{n}^{\prime}$. 
Para ver isto, considere $z^{1}$ tal que

$$
\left\langle p_{P^{\prime}}, z^{1}\right\rangle<0, \quad\left\langle p_{1}, z^{1}\right\rangle<0 \text { e }\left\langle p_{Q^{\prime}}, z^{1}\right\rangle>0
$$

e considere $z^{2}$ tal que

$$
\left\langle p_{P^{\prime}}, z^{2}\right\rangle<0, \quad\left\langle p_{1}, z^{2}\right\rangle>0 \text { e }\left\langle p_{Q^{\prime}}, z^{2}\right\rangle>0 .
$$

É claro que existe $z$ no segmento de reta $\left[z^{1}, z^{2}\right]$ tal que

$$
\left\langle p_{P^{\prime}}, z\right\rangle<0, \quad\left\langle p_{1}, z\right\rangle=0 \text { e }\left\langle p_{Q^{\prime}}, z\right\rangle>0
$$

Por convexidade, $\left\langle p_{P^{\prime}}^{\prime}, z\right\rangle<0$ e $\left\langle p_{Q^{\prime}}^{\prime}, z\right\rangle>0$, e portanto $\left(P^{\prime}, Q^{\prime}\right)$ define uma partição separável dos pontos projetados $p_{2}^{\prime}, \ldots, p_{n}^{\prime}$.

Como não podem haver mais do que duas partições induzidas idênticas, e além disso todos os pares de partições coincidentes definem partições separáveis (distintas) dos pontos projetados, o número de partições separáveis de $p_{1}, \ldots, p_{n}$ é limitado superiormente por $v_{m}(n-1)+v_{m-1}(n-1)$. Aqui $v_{m}(n-1)$ é o número máximo de partições separáveis de $p_{2}, \ldots, p_{n}$, e $v_{m-1}(n-1)$ é o número máximo de partições separáveis de $p_{2}^{\prime}, \ldots, p_{n}^{\prime}$. Ou seja, tem-se a relação de recorrência

$$
v_{m}(n) \leq v_{m}(n-1)+v_{m-1}(n-1), \forall(m, n): n>m
$$

Considere agora que os pontos $p_{1}, \ldots, p_{n}$ estão em posição geral. Então os pontos projetados $p_{2}^{\prime}, \ldots, p_{n}^{\prime}$ estão em posição geral com respeito ao hiperplano $\mathcal{H}$. Suponha que isto não é verdade: neste caso existiriam $m$ dentre os pontos projetados pertencentes a uma mesma variedade afim $m-2$ dimensional. Então estes $m$ pontos juntamente com $p_{1}$ são $m+1$ pontos em $\mathbb{R}^{m}$ pertencentes a uma mesma variedade afim $m$-1-dimensional, contradizendo a hipótese de que $p_{1}, \ldots, p_{n}$ estão em posição geral. Portanto 
na contagem anterior do número de partições separáveis, conclui-se que

$$
v_{m}(n)=v_{m}(n-1)+v_{m-1}(n-1)
$$

pois as quantidades $v_{m}(n-1)$ e $v_{m-1}(n-1)$ são o número exato de partições separáveis dos subconjuntos $\left\{p_{2}, \ldots, p_{n}\right\}$ e $\left\{p_{2}^{\prime}, \ldots, p_{n}^{\prime}\right\}$ de pontos em posição geral.

A solução (única) para a fórmula de recorrência

$$
\begin{cases}v_{m}(n)=2^{n-1}, & \text { se } n \leq m \\ v_{m}(n)=v_{m}(n-1)+v_{m-1}(n-1), & \text { se } n>m\end{cases}
$$

é dada por

$$
v_{m}(n)=\sum_{i=0}^{m}\left(\begin{array}{c}
n-1 \\
i
\end{array}\right)
$$

e isto prova a afirmação que $v_{m}(n)$ é um limitante superior para o número de partições separáveis de $n$ pontos em $\mathbb{R}^{m}$, que corresponde ao número exato de partições separáveis de $n$ pontos em posição geral em $\mathbb{R}^{m}$.

\section{Observação 1.8 (Hipótese de pontos em posição geral)}

Outro modo de ver que a hipótese de posição geral é uma hipótese de pior caso (maior número possível de partições separáveis) é observar que toda configuração de $n$ pontos em $\mathbb{R}^{m}$ pode ser minimamente perturbada de modo a se obter pontos em posição geral. Escolhendo o raio de perturbação $\varepsilon>0$ como

$$
\varepsilon=\frac{1}{2} \min \left\{d\left(A_{i}, A_{j}\right) \mid i \in P, j \in Q,(P, Q) \text { é partição separável }\right\},
$$

fica garantida a propriedade que toda partição separável da configuração original é também uma partição separável da configuração perturbada. Isso prova a afirmação.

Note que $v_{m}(n)=\Theta\left(n^{m}\right)$, ou seja, $v_{m}(n)$ é um polinômio em $n$ de grau $m$, para $m$ fixado. Esta observação leva à polinomialidade do método enumerativo 
apresentado na seção 1.3 , com respeito ao número $n$ de dados $\left(A_{i}, b_{i}\right)$, que é também o número de pares de variáveis complementares.

Como o número de possíveis partições de $\{1,2, \ldots, n\}$ é $2^{n-1}$, então pelo menos

$$
2^{n-1}-v_{m}(n)=\sum_{i=m+1}^{n-1}\left(\begin{array}{c}
n-1 \\
i
\end{array}\right)
$$

partições são não-separáveis, e este número é $\Theta\left(2^{n-1}\right)$ para $m$ fixado, ou seja, o número de partições não-separáveis cresce exponencialmente com $n$, para $m$ fixado. O restante desta seção analiza partições não-separáveis e as propriedades dos subproblemas $\left(\mathrm{HF}_{P Q}\right)$ correspondentes.

\section{Não-separabilidade e Degenerescência}

É natural considerar o que acontece ao problema $\left(\operatorname{HF}_{P Q}\right)$ se $(P, Q)$ é uma partição qualquer (não-separável). O lema a seguir fornece uma caracterização destas partições.

\section{Lema 1.9 (Partições não-separáveis geram problemas degenerados)}

$(P, Q)$ é uma partição não-separável se, e somente se, o problema $\left(H F_{P Q}\right)$ é degenerado, no sentido que existe um subconjunto de índices $\bar{Q} \subset Q, \bar{Q} \neq \emptyset$, tal que $r_{\bar{Q}}=s_{\bar{Q}}=0$ em todas as soluções viáveis de $\left(H F_{P Q}\right)$.

Prova : Como visto anteriormente, todos os problemas $\left(\mathrm{HF}_{P Q}\right)$ são viáveis e possuem soluções ótimas. Mais ainda, para toda solução viável $(x, y)$ em $\left(\mathrm{HF}_{P Q}\right)$, as desigualdades $A_{P} x \geq A_{P} y$ e $A_{Q} x \leq A_{Q} y$ são válidas.

Se, por um lado, $(P, Q)$ é uma partição não-separável dos pontos dados, então não existe nenhum par $(x, y)$ tal que $A_{P} x \geq A_{P} y$ e $A_{Q} x<A_{Q} y$. Juntamente com as desigualdades anteriores, segue que para toda so- 
lução $(x, y)$ viável em $\left(\mathrm{HF}_{P Q}\right)$, existe ao menos um índice $j \in Q$ tal que $A_{j} x \geq A_{j} y$ e, lembrando que $A_{Q} x \leq A_{Q} y$, tem-se $A_{j} x=A_{j} y$.

Pela convexidade do conjunto viável de $\left(\mathrm{HF}_{P Q}\right)$, e pela finitude de $Q$, conclui-se que o conjunto

$$
\bar{Q}=\left\{j \in Q \mid A_{j} x=A_{j} y \text { para toda solução viável }(x, y) \text { em }\left(\mathrm{HF}_{P Q}\right)\right\}
$$

é não-vazio. Suponha, por contradição, que $\bar{Q}=\emptyset$. Então para todo $j \in Q$ existiria uma par $\left(x^{j}, y^{j}\right)$ viável em $\left(\mathrm{HF}_{P Q}\right)$ tal que $A_{j} x^{j}<A_{j} y^{j}$. Isto implica que a combinação convexa $(x, y)=\frac{1}{|Q|} \sum_{j \in Q}\left(x^{j}, y^{j}\right)$ satisfaz $A_{P} x \geq A_{P} y$ e $A_{Q} x<A_{Q} y$, contradizendo a não-separabilidade de $(P, Q)$. Portanto $\bar{Q} \neq \emptyset$.

Do comentário acima, segue que todas as soluções viáveis do problema $\left(\mathrm{HF}_{P Q}\right)$ satisfazem $r_{j}=s_{j}=0$, para todo $j \in \bar{Q}$, e são portanto degeneradas.

Suponha, por outro lado, que exista um subconjunto de índices $\bar{Q} \subset Q$, $\bar{Q} \neq \emptyset$, tal que $r_{\bar{Q}}=s_{\bar{Q}}=0$ em todas as soluções viáveis de $\left(H_{P Q}\right)$. Então, para toda solução $(x, y)$ viável em $\left(\mathrm{HF}_{P Q}\right)$, tem-se $A_{\bar{Q}} x=A_{\bar{Q}} y$, e portanto não pode existir $(x, y)$ tal que $A_{Q} x<A_{Q} y$. Deste modo, a partição $(P, Q)$ não é separável.

Uma das possíveis conseqüências de degenerescência, no caso em que $A_{\bar{Q}}$ tem característica plena, é o colapso do problema $\left(\mathrm{HF}_{P Q}\right)$ a um problema de regressão linear comum, pois neste caso $A_{\bar{Q}} x=A_{\bar{Q}} y \Longrightarrow x=y$. Os problemas $\left(\mathrm{HF}_{P Q}\right)$ nãotriviais, isto é, aqueles que não se reduzem a um problema de regressão linear, são determinados crucialmente pela configuração geométrica dos pontos $A_{P}$ e $A_{Q}$.

A degenerescência desaparece, por exemplo, ao definir-se $P^{\prime}=P \cup \bar{Q}$ e $Q^{\prime}=Q \backslash \bar{Q}$. Então obtém-se uma partição separável $\left(P^{\prime}, Q^{\prime}\right)$, pelo argumento de 
convexidade acima. O conjunto viável de $\left(\mathrm{HF}_{P Q}\right)$ está contido trivialmente naquele de $\left(\mathrm{HF}_{P^{\prime} Q^{\prime}}\right)$, de modo que vale a pena considerar apenas o problema não-degenerado $\left(\mathrm{HF}_{P^{\prime} Q^{\prime}}\right)$.

É importante lembrar que toda solução viável de (HF) é também imediatamente uma solução viável de pelo menos um subproblema $\left(\mathrm{HF}_{P Q}\right)$ definido por uma partição separável $(P, Q)$. No restante deste trabalho, serão consideradas apenas partições separáveis.

\subsection{O Algoritmo para Obtenção de Dobradiça (HFA)}

A primeira abordagem para resolver o problema de Ajuste de Dobradiças, proposta por Breiman [Breiman 1993], tenta encontrar uma partição separável que seja um ponto fixo da função

$$
\varphi(P, Q)=\left(P^{\varphi}, Q^{\varphi}\right)
$$

onde $P^{\varphi}=\left\{k \mid A_{k} x^{\varphi} \geq A_{k} y^{\varphi}\right\}, Q^{\varphi}=\left\{k \mid A_{k} x^{\varphi}<A_{k} y^{\varphi}\right\}, x^{\varphi}$ é a solução do problema de regressão linear aplicado aos dados $\left\{\left(A_{i}, b_{i}\right)\right\}_{i \in P}$, e $y^{\varphi}$ é a solução do problema de regressão linear aplicado aos dados $\left\{\left(A_{j}, b_{j}\right)\right\}_{j \in Q}$.

\section{$\mathrm{O}$ algoritmo (HFA)}

0. Seja $(P, Q)$ uma partição separável, e seja $\left(P^{\varphi}, Q^{\varphi}\right) \leftarrow \varphi(P, Q)$

1. Enquanto $(P, Q) \neq\left(P^{\varphi}, Q^{\varphi}\right)$ faça

$$
\begin{aligned}
& (P, Q) \leftarrow\left(P^{\varphi}, Q^{\varphi}\right), \mathrm{e} \\
& \left(P^{\varphi}, Q^{\varphi}\right) \leftarrow \varphi(P, Q) .
\end{aligned}
$$




\section{Observação 1.10 (Solução dos subproblemas de regressão linear)}

Note que $x^{\varphi}$ e $y^{\varphi}$ são, por definição, as soluçôes ótimas dos problemas

$$
\begin{aligned}
& \min \frac{1}{2}\left\|A_{P} x-b_{P}\right\|^{2}, \\
& \min \frac{1}{2}\left\|A_{Q} y-b_{Q}\right\|^{2} .
\end{aligned}
$$

Como estes são problemas quadráticos convexos, as condições de otimalidade de primeira ordem $\nabla_{x} \frac{1}{2}\left\|A_{P} x-b_{P}\right\|^{2}=0$ e $\nabla_{y} \frac{1}{2}\left\|A_{Q} y-b_{Q}\right\|^{2}=0$ são necessárias e suficientes. Estas condições são

$$
\begin{aligned}
& A_{P}^{t} A_{P} x=A_{P}^{t} b_{P} \\
& A_{Q}^{t} A_{Q} y=A_{Q}^{t} b_{Q} .
\end{aligned}
$$

Quando as matrizes $A_{P}^{t} A_{P}$ e $A_{Q}^{t} A_{Q}$ são não-singulares, a solução única é dada pela fórmula fechada

$$
\left[\begin{array}{l}
x^{\varphi} \\
y^{\varphi}
\end{array}\right]=\left[\begin{array}{c}
\left(A_{P}^{t} A_{P}\right)^{-1} A_{P}^{t} b_{P} \\
\left(A_{Q}^{t} A_{Q}\right)^{-1} A_{Q}^{t} b_{Q}
\end{array}\right] .
$$

Lema 1.11 (Breiman 1993) Se $\left(P^{*}, Q^{*}\right)$ é um ponto fixo da função $\varphi(\cdot, \cdot)$, associado à solução $\left(x^{*}, y^{*}\right)$, então $\left(x^{*}, y^{*}\right)$ é um minimo local do problema

$$
\min _{x, y} \sum_{i=1}^{n}\left(\max \left\{A_{i} x, A_{i} y\right\}-b_{i}\right)^{2} .
$$

Prova : A prova completa pode ser encontrada em [Breiman 1993]. Considera-se aqui o caso em que $\left(P^{*}, Q^{*}\right)$ e $\left(x^{*}, y^{*}\right)$ satisfazem

$$
\begin{aligned}
& P^{*}=\left\{k \mid A_{k} x^{*}>A_{k} y^{*}\right\} \\
& Q^{*}=\left\{k \mid A_{k} x^{*}<A_{k} y^{*}\right\},
\end{aligned}
$$

ou seja, que $\left(x^{*}, y^{*}\right)$ separa estritamente $\left(P^{*}, Q^{*}\right)$. Neste caso, existe uma vizinhança $U$ de $\left(x^{*}, y^{*}\right)$ tal que, para qualquer $(x, y) \in U$, a partição induzida satisfaz $(P(x, y), Q(x, y))=\left(P^{*}, Q^{*}\right)$. Então, para qualquer 


$$
\begin{aligned}
& (x, y) \in U, \\
& \quad \sum_{i=1}^{n}\left(\max \left\{A_{i} x, A_{i} y\right\}-b_{i}\right)^{2}=\sum_{i \in P^{*}}\left(A_{i} x-b_{i}\right)^{2}+\sum_{i \in Q^{*}}\left(A_{i} y-b_{i}\right)^{2} .
\end{aligned}
$$

Portanto a função objetivo é convexa e separável, e cada uma das somatórias corresponde à função objetivo do problema de regressão linear associado a $\left\{\left(A_{i}, b_{i}\right)\right\}_{i \in P^{*}}$ e $\left\{\left(A_{i}, b_{i}\right)\right\}_{i \in Q^{*}}$, respectivamente. Pela otimalidade local de $x^{*}$ e $y^{*}$ naqueles problemas, existem vizinhanças $U_{x}$ e $U_{y}$ de $x^{*}$ e $y^{*}$, respectivamente, tais que, para todo $(x, y) \in U_{x} \times U_{y}$,

$$
\begin{aligned}
& \sum_{i \in P^{*}}\left(A_{i} x^{*}-b_{i}\right)^{2} \leq \sum_{i \in P^{*}}\left(A_{i} x-b_{i}\right), \mathrm{e} \\
& \sum_{i \in Q^{*}}\left(A_{i} y^{*}-b_{i}\right)^{2} \leq \sum_{i \in Q^{*}}\left(A_{i} y-b_{i}\right)^{2} .
\end{aligned}
$$

Portanto, para todo $(x, y) \in U \cap\left(U_{x} \times U_{y}\right)$,

$$
\sum_{i=1}^{n}\left(\max \left\{A_{i} x^{*}, A_{i} y^{*}\right\}-b_{i}\right)^{2} \leq \sum_{i=1}^{n}\left(\max \left\{A_{i} x, A_{i} y\right\}-b_{i}\right)^{2} .
$$

Um exemplo de ponto fixo que é mínimo local, mas não é mínimo global para o problema, é indicado na figura abaixo, à esquerda. À direita, é indicado o mínimo global para o mesmo problema. Os conjuntos $P$ e $Q$ estão indicados no eixo horizontal.
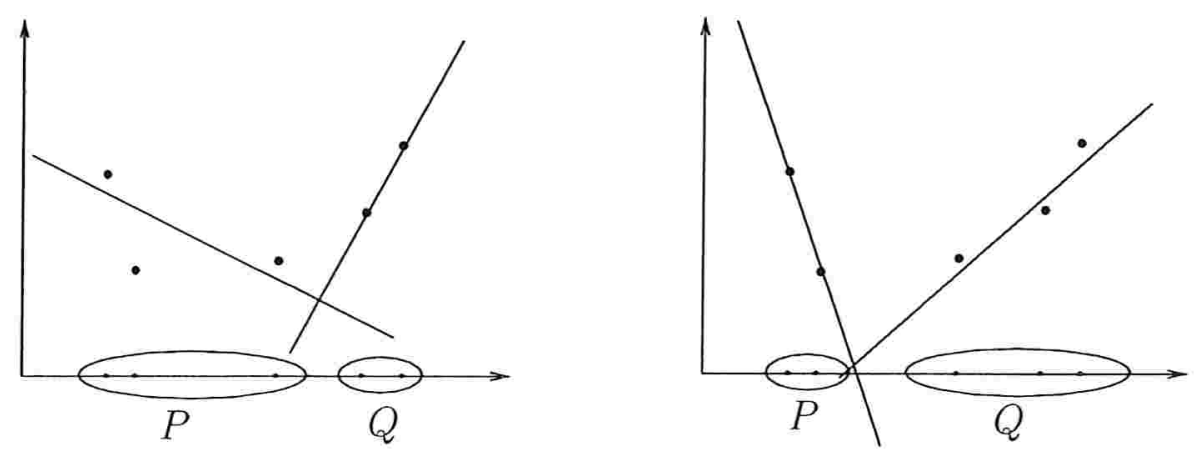
Um dos aspectos estranhos do algoritmo é a presença de um ponto fixo anômalo, dado pela partição trivial $(\{1,2, \ldots, n\}, \emptyset)$. Não é infreqüente o algoritmo atingir esta partição trivial ao tentar aproximar funções que não são dobradiças, ou mesmo ao tentar aproximar dobradiças, partindo de uma solução inicial distante.

Talvez um dos problemas mais sérios do algoritmo seja a possibilidade de ciclagem [Pucar \& Sjöberg 1998], e a dificuldade computacional em identificar este caso. Pode-se observar ciclagem em exemplos muito simples, como os dados pelas duas figuras a seguir. Nestes exemplos, as partições $(P, Q)$ indicadas no eixo horizontal são aquelas utilizadas para resolver os subproblemas de regressão linear.
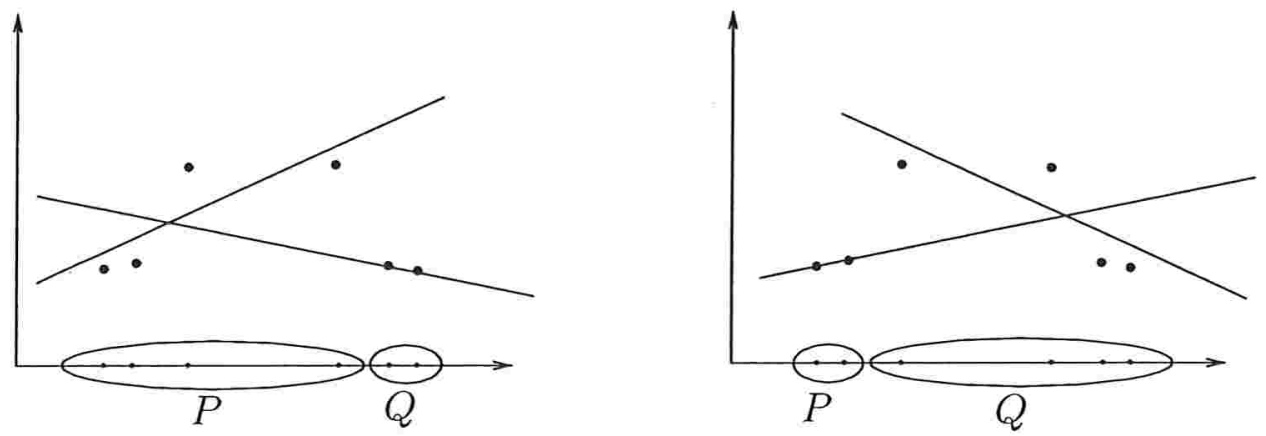

A dificuldade computacional em identificar ciclagem deve-se, em parte, ao fato de que a função de mérito natural $\|\operatorname{vecmax}\{A x, A y\}-b\|$ não é utilizada pelo algoritmo.

Para resolver estes problemas, uma modificação foi proposta em [Pucar \& Sjöberg 1998], que corresponde a realizar uma busca linear entre a dobradiça dada por $(x, y)$, correspondente a $(P, Q)$, e a nova dobradiça definida por $\varphi$, dada por $\left(x^{\varphi}, y^{\varphi}\right)$. A busca linear visa a uma solução $\left(x^{\lambda}, y^{\lambda}\right)=\lambda(x, y)+(1-\lambda)\left(x^{\varphi}, y^{\varphi}\right)$ tal que

$$
\left\|\operatorname{vecmax}\left\{A x^{\lambda}, A y^{\lambda}\right\}-b\right\|<\|\operatorname{vecmax}\{A x, A y\}-b\|
$$

A estratégia proposta em [Pucar \& Sjöberg 1998] para escolha do tamanho de passo é tentar $\lambda=1, \frac{1}{2}, \frac{1}{4}, \ldots$ e tomar o primeiro valor que satisfaz o critério de descida 
acima. Esta é uma dentre várias escolhas possíveis que garantem convergência global, ou seja, convergência a partir de qualquer ponto inicial para uma solução localmente ótima do (HF) [Pucar \& Sjöberg 1998].

\section{Observação 1.12 (HFA como método de Newton)}

Esta modificação pode ser interpretada como um amortecimento de passo no contexto do método de Newton. Para ver que o método original de Breiman é um método do tipo Newton, considere a formulação original do problema (HF)

$$
\min _{x, y} \Phi(x, y)=\frac{1}{2}\|h(A, x, y)-b\|^{2}
$$

onde $h(A, x, y)=\operatorname{vecmax}\{A x, A y\}$. Considere que $(x, y)$ é uma solução tal que $A_{k} x \neq A_{k} y$, para todo índice $k$. Então pode-se escrever as derivadas parciais de $h$ como

$$
\begin{aligned}
& \frac{\partial h(A, x, y)}{\partial x}=\left[\frac{A_{P}}{0}\right] \\
& \frac{\partial h(A, x, y)}{\partial y}=\left[\frac{A_{Q}}{0}\right]
\end{aligned}
$$

onde as linhas não-nulas correspondem aos indices em $P$ e $Q$, respectivamente.

Então o gradiente de $\Phi$ é dado por

$$
\nabla \Phi=\left[\begin{array}{c}
\frac{\partial \Phi}{\partial x} \\
\frac{\partial \Phi}{\partial y}
\end{array}\right]=\left[\begin{array}{c}
\frac{\partial h(A, x, y)}{\partial x}(h(A, x, y)-b) \\
\frac{\partial h(A, x, y)}{\partial y}(h(A, x, y)-b)
\end{array}\right]=\left[\begin{array}{c}
A_{P}^{t} A_{P} x-A_{P}^{t} b_{P} \\
A_{Q}^{t} A_{Q} y-A_{Q}^{t} b_{Q}
\end{array}\right]
$$

e, notando que $P \cap Q=\emptyset$ e $\log o \frac{\partial^{2} \Phi}{\partial x \partial y}=\frac{\partial^{2} \Phi}{\partial y \partial x}=0$, segue que

$$
\nabla^{2} \Phi=\left[\begin{array}{cc}
A_{P}^{t} A_{P} & 0 \\
0 & A_{Q}^{t} A_{Q}
\end{array}\right]
$$


Supondo que a Hessiana $\nabla^{2} \Phi$ é não-singular, é possivel aplicar um passo de Newton a partir de $(x, y)$, e o novo ponto $\left(x^{N}, y^{N}\right)$ obedecerá

$$
\begin{aligned}
{\left[\begin{array}{l}
x^{N} \\
y^{N}
\end{array}\right] } & =\left[\begin{array}{l}
x \\
y
\end{array}\right]+\left[\begin{array}{cc}
A_{P}^{t} A_{P} & 0 \\
0 & A_{Q}^{t} A_{Q}
\end{array}\right]^{-1}\left[\begin{array}{c}
A_{P}^{t} b_{P}-A_{P}^{t} A_{P} x \\
A_{Q}^{t} b_{Q}-A_{Q}^{t} A_{Q} y
\end{array}\right] \\
& =\left[\begin{array}{l}
x \\
y
\end{array}\right]+\left(\left[\begin{array}{l}
x^{\varphi} \\
y^{\varphi}
\end{array}\right]-\left[\begin{array}{l}
x \\
y
\end{array}\right]\right) \\
& =\left[\begin{array}{l}
x^{\varphi} \\
y^{\varphi}
\end{array}\right] .
\end{aligned}
$$

A modificação de Pucar \&S Sjöberg corresponde a amortecer este passo, fazendo

$$
\left[\begin{array}{l}
x^{N} \\
y^{N}
\end{array}\right]=\left[\begin{array}{l}
x \\
y
\end{array}\right]+(1-\lambda)\left(\left[\begin{array}{l}
x^{\varphi} \\
y^{\varphi}
\end{array}\right]-\left[\begin{array}{l}
x \\
y
\end{array}\right]\right),
$$

e com isso se insere no contexto de métodos de Newton amortecidos (vide, por exemplo, [Stoer \& Burlisch 1992], seção 5.4).

\subsection{Um Método Enumerativo para o (HF)}

O objetivo desta seção é apresentar um método enumerativo para o problema de Ajuste Euclideano de Dobradiças, que concentra-se em subproblemas $\left(\mathrm{HF}_{P Q}\right)$ para partições separáveis $(P, Q)$ dos pontos dados.

Definição 1.13 (Subpartição separável)

$(I, J)$ é uma subpartição separável de $\{1,2, \ldots, n\}$ se $I \cup J \subseteq\{1,2, \ldots, n\}$, $I \cap J=\emptyset e(I, J)$ é uma partição separável de $I \cup J$. Dada uma subpartição $(I, J)$ o complemento $K(I, J)$ é definido como $K(I, J)=\{1,2, \ldots, n\} \backslash(I \cup J)$. Quando o contexto for claro, a notação $K \equiv K(I, J)$ é adotada. 
A definição do problema $\left(\mathrm{HF}_{P Q}\right)$ é estendida naturalmente às subpartições separáveis $(I, J)$, ao considerar-se a condição de complementaridade restrita aos índices em $K(I, J)$, como segue

$$
\left(\mathrm{HF}_{I J}\right) \begin{cases}\min & \sigma^{t} \sigma \\ \text { s.a. } & r=\sigma-A x+b \\ & s=\sigma-A y+b \\ & r_{I}=0 \\ & s_{J}=0 \\ & r, s \geq 0 \\ & r_{K}^{t} s_{K}=0\end{cases}
$$

Uma abordagem enumerativa geral para resolver o (HF) é apresentada a seguir, para referência. O algoritmo constrói uma árvore de enumeração (implícita)

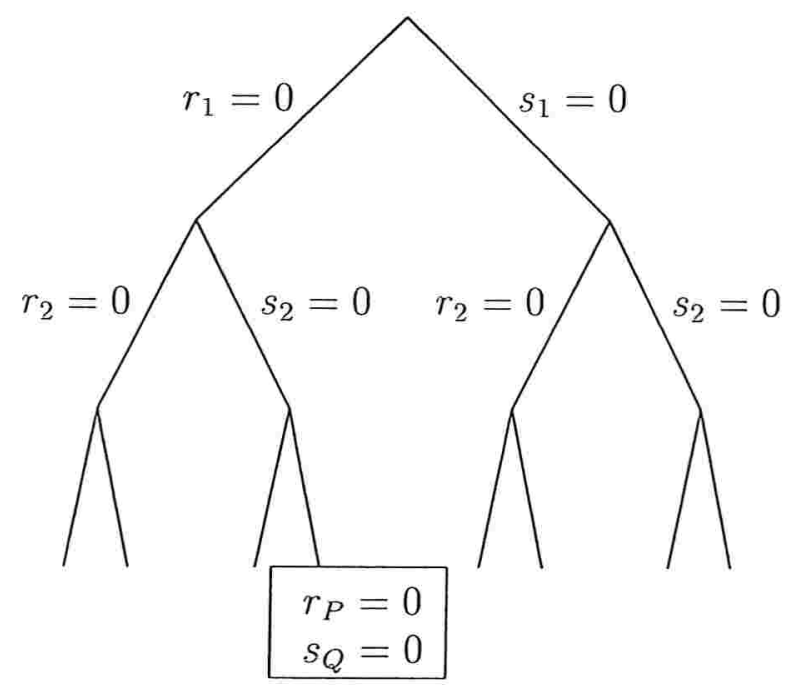

onde os nós interiores correspondem a problemas $\left(\mathrm{HF}_{I J}\right)$ para subpartições separáveis $(I, J)$, e as folhas da árvore correspondem a partições separáveis. A enumeração é dita implícita porque várias sub-árvores são excluídas da busca em função das estimativas (limitantes inferiores e superiores) computadas em cada nó. É claro que $(\mathrm{HF})=\left(\mathrm{HF}_{\emptyset \emptyset}\right)$, 
de modo que o primeiro nó a ser processado é o $(\emptyset, \emptyset)$. A discussão detalhada de passos importantes do algoritmo é apresentada na seqüência.

\section{Um algoritmo enumerativo para o (HF)}

0. (inicialização) Faça

$\mathcal{L} \longleftarrow\{(\emptyset, \emptyset)\}:$ Lista de nós a serem investigados, e

$U B \longleftarrow \infty$ : Melhor limitante superior (Upper Bound) viável (i.e. associado a uma solução viável) conhecido.

1. Se $\mathcal{L}=\emptyset$ pare! O valor ótimo é dado por $U B$.

Caso contrário, escolha $(I, J) \in \mathcal{L}$, e faça

$\mathcal{L} \longleftarrow \mathcal{L} \backslash(I, J)$.

2. (opcional) Seja

$$
\begin{aligned}
& I \longleftarrow I \cup\left\{k \in K \mid r_{k} \equiv 0 \mathrm{em}\left(\mathrm{HF}_{I J}\right)\right\} \mathrm{e} \\
& J \longleftarrow J \cup\left\{k \in K \mid s_{k} \equiv 0 \mathrm{em}\left(\mathrm{HF}_{I J}\right)\right\} .
\end{aligned}
$$

3. Compute um limitante inferior $L B$ (Lower Bound) para $\left(\mathrm{HF}_{I J}\right)$, resolvendo o problema relaxado

$$
\left(L B_{I J}\right) \begin{cases}\min & \sigma^{t} \sigma \\ \text { s.a. } & r=\sigma-A x+b \\ & s=\sigma-A y+b \\ & r_{I}=0 \\ & s_{J}=0 \\ & r, s \geq 0\end{cases}
$$

Se o limitante inferior obtido satisfaz $r_{K}^{t} s_{K}=0$, faça $U B \longleftarrow \min \{U B, L B\}$. 
Se $L B \geq U B$ volte ao passo 1 .

4. (opcional) Compute novos limitantes superiores $U B_{1}, \ldots, U B_{p}$ viáveis para $\left(\mathrm{HF}_{I J}\right)$, para um dado $p \in \mathbb{N}$. Faça

$U B \longleftarrow \min \left\{U B, U B_{1}, \ldots, U B_{p}\right\}$.

5. Ramificação: escolha $k \in K$ tal que $(I \cup\{k\}, J)$ e $(I, J \cup\{k\})$ sejam subpartições separáveis, e faça

$\mathcal{L} \longleftarrow \mathcal{L} \cup\{(I \cup\{k\}, J),(I, J \cup\{k\})\}$.

Volte ao passo 1.

O método apresentado segue uma estratégia branch-and-bound (ramificar e limitar), logo os pontos principais são a escolha do próximo nó da árvore a ser processado, o cômputo de limitantes inferiores e superiores e a estratégia de ramificação. Outro ponto importante é a possibilidade de fixação de variáveis adicionais no passo 2, que depende da geometria das subpartições separáveis. Cada um dos passos do algoritmo será agora abordado, em ordem.

\section{Escolha do Próximo Nó}

Um importante problema de implementação é a escolha do novo nó da árvore a ser processado depois de um passo de ramificação. A política FIFO (First-In-First-Out) trata a lista de nós como uma fila, e leva a uma exploração em largura da árvore; esta é uma boa escolha para obter rapidamente bons limitantes inferiores. A política LIFO (Last-In-First-Out) corresponde a uma implementação da lista de nós como pilha, e leva a uma busca em profundidade que almeja rapidamente limitantes superiores viáveis. Outras estratégias mistas também podem ser usadas. Na experiência computacional aqui apresentada, a estratégia de busca em largura levou a um melhor desempenho, e é usada como opção padrão. 


\section{A Geometria das Subpartiçōes Separáveis}

O passo 2 do algoritmo tenta aumentar os conjuntos $I$ e $J$ que formam a subpartição separável, identificando índices $k \in K$ tais que $r_{k}=0$ ou $s_{k}=0$ para toda solução viável de $\left(\mathrm{HF}_{I J}\right)$. Considere os conjuntos

$$
\begin{aligned}
& \mathcal{R}_{I J}=\left\{a \mid a^{t} x \geq a^{t} y, \forall(x, y) \text { viável em }\left(\operatorname{HF}_{I J}\right)\right\} \\
& \mathcal{S}_{I J}=\left\{a \mid a^{t} y \geq a^{t} x, \forall(x, y) \text { viável em }\left(\operatorname{HF}_{I J}\right)\right\}
\end{aligned}
$$

O próximo resultado relaciona estes conjuntos aos índices $k \in K$ tais que $r_{k}=0$ ou $s_{k}=0$ vale necessariamente em $\left(\mathrm{HF}_{I J}\right)$.

\section{Lema 1.14 (Caracterização de $\mathcal{R}_{I J}$ e $\mathcal{S}_{I J}$ )}

Seja $(r, s, x, y, \sigma)$ uma solução viável de $\left(H F_{I J}\right)$. Se $A_{k} \in \mathcal{R}_{I J}$, então $r_{k}=0$; se $A_{k} \in \mathcal{S}_{I J}$, então $s_{k}=0$.

Prova: Se $A_{k} \in \mathcal{R}_{I J}$, então $A_{k} x \geq A_{k} y$, e portanto $s_{k} \geq r_{k}$, de onde $r_{k}=0$, por complementaridade. A implicação $A_{k} \in \mathcal{S}_{I J} \Longrightarrow s_{k}=0$ é derivada de maneira análoga.

Uma conseqüência direta deste resultado é a seguinte.

Proposiçāo 1.15 (Equivalência entre $\left(\mathrm{HF}_{I J}\right)$ e $\left.\left(\mathrm{HF}_{\hat{I} \hat{J}}\right)\right)$

$\left(H F_{I J}\right)$ é equivalente a $\left(H F_{\hat{I} \hat{J}}\right)$, onde

$$
\begin{aligned}
& \hat{I}=\left\{k \mid A_{k} \in \mathcal{R}_{I J}\right\} \\
& \hat{J}=\left\{k \mid A_{k} \in \mathcal{S}_{I J}\right\} .
\end{aligned}
$$


Com este resultado, pode-se reformular o passo 2 do algoritmo como

2'. Faça $I \longleftarrow \hat{I}$ e $J \longleftarrow \hat{J} \backslash \hat{I}$, onde $(\hat{I}, \hat{J})$ são dados pela proposição 1.15 .

A definição dos conjuntos $\mathcal{R}_{I J}$ e $\mathcal{S}_{I J}$ conduz a um teste natural de pertinência: se o Problema Linear (PL)

$$
\begin{cases}\min & A_{k} x-A_{k} y \\ \text { s.a. } & A_{I} x \geq A_{I} y \\ & A_{J} y \geq A_{J} x\end{cases}
$$

tem valor ótimo não-negativo, então $A_{k} \in \mathcal{R}_{I J}$. Por outro lado, se $A_{k} \notin \mathcal{R}_{I J}$, então existe algum par $(x, y)$ viável tal que $A_{k} x-A_{k} y<0$, e portanto o problema acima é ilimitado. Substituindo $z=x-y$ obtém-se a seguinte propriedade.

\section{Lema 1.16 (Teste computacional para $A_{k} \in \mathcal{R}_{I J}$ )}

$A_{k} \in \mathcal{R}_{I J}$ se, e somente se, o $P L$

$$
\begin{cases}\min & A_{k} z \\ \text { s.a. } & A_{I} z \geq 0 \\ & A_{J} z \leq 0\end{cases}
$$

tem valor ótimo não-negativo. Um teste análogo para $\mathcal{S}_{I J}$ é obtido substituindo-se min por $\max$ no PL acima.

O passo 2 do algoritmo pode ser implementado como segue. Primeiro resolvese um PL para testar se $A_{k} \in \mathcal{R}_{I J}$, e o conjunto $I$ é atualizado, se necessário. Se $A_{k} \notin \mathcal{R}_{I J}$, e somente neste caso, um PL é resolvido para testar se $A_{k} \in \mathcal{S}_{I J}$, e o conjunto $J$ é atualizado se necessário. Isso impede que o mesmo índice $k$ seja introduzido nos dois conjuntos $I$ e $J$, no caso especial em que $A_{k} \in \mathcal{R}_{I J} \cap \mathcal{S}_{I J}$. Portanto são resolvidos no máximo dois PLs para cada $k \in K$.

Do ponto de vista pragmático, o passo 2 do algoritmo representa uma grande sobrecarga computacional quando $I$ e $J$ são pequenos, devido a.o fato que, neste caso, 
a maioria dos pontos $A_{k}$ não estão nem em $\mathcal{R}_{I J}$ nem em $\mathcal{S}_{I J}$, e o tempo necessário para resolver os PLs acima é desperdiçado. Por outro lado, se $K$ é pequeno comparado a $I$ e $J$, este teste pode evitar ramificações desnecessárias nos nós próximos às folhas.

\section{Observação 1.17 (Ilustração do lema 1.14 e da proposição 1.15)}

É fácil ver que a representação dual dos conjuntos $\mathcal{R}_{I J}$ e $\mathcal{S}_{I J}$ é

$$
\begin{aligned}
& \mathcal{R}_{I J}=\operatorname{conv}\left\{A_{i}\right\}_{i \in I}+\operatorname{cone}\left\{A_{i}-A_{j}\right\}_{i \in I, j \in J}, \\
& \mathcal{S}_{I J}=\operatorname{conv}\left\{A_{j}\right\}_{j \in J}+\operatorname{cone}\left\{A_{j}-A_{i}\right\}_{j \in J, i \in I} .
\end{aligned}
$$

Esta representação pode ilustrar melhor o resultado do lema 1.14 e da proposição 1.15, como no exemplo a seguir.

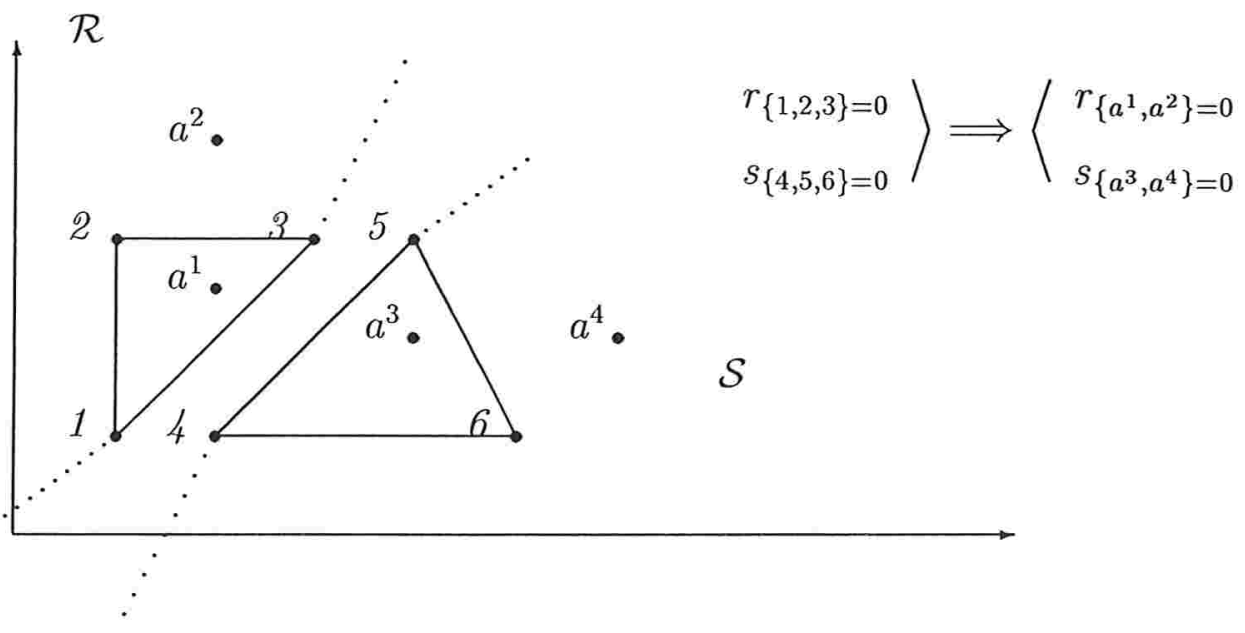

\section{Cômputo de Limitantes Inferiores e Superiores .}

Uma escolha natural para os limitantes inferiores dos subproblemas $\left(\mathrm{HF}_{I J}\right)$ é o problema relaxado $\left(L B_{I J}\right)$, obtido descartando-se a restrição de complementaridade. Este é um problema de programação quadrática convexo que pode ser resolvido por uma variedade de métodos. 
É imediato que o (HF) relaxado, ou $\left(L B_{\emptyset \emptyset}\right)$, tem uma solução trivial com $\sigma=0$, fazendo-se $x=y=(0, \ldots, 0, \bar{b})^{t}$ e $r=s=b-(\bar{b}, \ldots, \bar{b})^{t}$. O mesmo, porém, não é verdade acerca dos problemas relaxados $\left(L B_{I J}\right)$ em geral. Por exemplo, se $I=\{1,2, \ldots, n\}$ e $J=\emptyset$, então o problema $\left(L B_{I J}\right)=\left(\mathrm{HF}_{I J}\right)$ se reduz a um problema de regressão linear que, em geral, não possui solução com erro de aproximação $\sigma=0$.

As soluções de $\left(L B_{I J}\right)$ são em geral inviáveis para o problema $\left(\mathrm{HF}_{I J}\right)$; quando viáveis, estas também são ótimas para $\left(H_{I J}\right)$. Isto explica a atualização de $U B$ no passo 3 do algoritmo, que leva necessariamente à verificação da condição $L B \geq U B$, e à poda da árvore.

A condição $L B \geq U B$ poda a árvore, ao identificar subproblemas cuja solução não vale a pena conhecer, porque a solução ótima de $\left(\mathrm{HF}_{I J}\right)$ neste caso é pior do que alguma solução viável já conhecida. A eficiência de qualquer método enumerativo depende fortemente de quantas vezes esta condição é satisfeita durante a sua execução. Este número depende da obtenção de limitantes inferiores e superiores justos.

Como os subproblemas $\left(\mathrm{HF}_{I J}\right)$ são em geral tão difíceis de resolver quanto o problema original, bons limitantes superiores viáveis são normalmente obtidos por métodos heurísticos.

Considere uma solução ótima do subproblema $\left(L B_{I J}\right)$, e um dentre os índices $k \in K=\{1,2, \ldots, n\} \backslash(I \cup J)$. Como $\left(L B_{I J}\right)$ minimiza $\sigma_{k}^{2}$ e a única restrição que se aplica a $\sigma_{k}$ é $\sigma_{k} \geq \max \left\{A_{k} x, A_{k} y\right\}-b_{k}$, segue que só há duas situações possíveis:

1. $\max \left\{A_{k} x, A_{k} y\right\}-b_{k}<0$; neste caso $\sigma_{k}=0$ e $r_{k} s_{k}>0$.

2. $\max \left\{A_{k} x, A_{k} y\right\}-b_{k} \geq 0$; neste caso $\sigma_{k}=\max \left\{A_{k} x, A_{k} y\right\}-b_{k}$ e $r_{k} s_{k}=0$.

Portanto um certo número das restrições de complementaridade que foram descartadas pelo problema relaxado $\left(L B_{I J}\right)$ são satisfeitas implicitamente por causa da forma da função objetivo. Este comentário prova o seguinte fato. 
Lema 1.18 (Hereditariedade de solução ótima de $\left(L B_{I J}\right)$ )

Seja $(r, s, x, y, \sigma)$ uma solução ótima de $\left(L B_{I J}\right)$, e defina

$$
\begin{aligned}
& I^{\prime}=I \cup\left\{k \mid r_{k}=0\right\} \\
& J^{\prime}=J \cup\left\{k \mid s_{k}=0\right\} .
\end{aligned}
$$

Então $(r, s, x, y, \sigma)$ é também solução ótima de $\left(L B_{I^{\prime} J^{\prime}}\right)$.

Note a relação deste resultado com a proposição 1.15. Aqui os problemas $\left(L B_{I J}\right)$ e $\left(L B_{I^{\prime} J^{\prime}}\right)$ não são equivalentes, pois na definição de $I^{\prime}$ e $J^{\prime}$ são considerados os índices $k$ tais que $r_{k}=0$ ou $s_{k}=0$ na solução ótima de $\left(L B_{I J}\right)$, enquanto na proposição 1.15 a definição de $\hat{I}$ e $\hat{J}$ considera os índices $k$ tais que $r_{k} \equiv 0$ ou $s_{k} \equiv 0$ em todas as soluções viáveis de $\left(\mathrm{HF}_{I J}\right)$.

A solução do problema $\left(L B_{I^{\prime} J^{\prime}}\right)$ acima pode ser utilizada facilmente para se obter um limitante superior para os problemas $\left(\mathrm{HF}_{I^{\prime} J^{\prime}}\right)$ e $\left(\mathrm{HF}_{I J}\right)$, fazendo-se $\sigma_{K^{\prime}}=\operatorname{vecmax}\left\{A_{K^{\prime}} x, A_{K^{\prime}} y\right\}-b_{K^{\prime}}$, onde $K^{\prime}=\{1,2, \ldots, n\} \backslash\left(I^{\prime} \cup J^{\prime}\right)$. O limitante superior assim obtido é $L B_{I J}+\sigma_{K^{\prime}}^{t} \sigma_{K^{\prime}}$.

Um limitante superior melhor do que o anterior pode ser obtido eficientemente utilizando-se a solução de $\left(L B_{I^{\prime} J^{\prime}}\right)$ como entrada para o algoritmo HFA de Pucar \& Breiman, apresentado na seção 1.2. Além disso o limitante superior do passo 0 pode ser obtido pela solução do HFA com uma entrada qualquer.

Dependendo da qualidade destes limitantes inferiores e superiores, novos limitantes superiores podem ser obtidos resolvendo-se novos problemas de programação quadrática. As atribuições $I^{\prime \prime}=I^{\prime} \cup K^{\prime}$ e $J^{\prime \prime}=J^{\prime} \cup K^{\prime}$ levam a duas possíveis escolhas para os limitantes superiores, quais sejam, as soluções dos problemas quadráticos convexos $\left(\mathrm{HF}_{I^{\prime \prime} J^{\prime}}\right)$ e $\left(\mathrm{HF}_{I^{\prime} J^{\prime \prime}}\right)$. 


\section{Ramificação}

O último passo do algoritmo consiste em escolher alguma variável $k \in K$, e acrescentar dois subproblemas à lista, correspondentes às subpartições $(I \cup\{k\}, J)$ e $(I, J \cup\{k\})$. Do ponto de vista teórico, qualquer escolha do índice de ramificação garante a corretude do algoritmo. No entanto, o desempenho do algoritmo é sensível a esta escolha. Uma heurística boa (empiricamente) consiste em escolher $k=\operatorname{argmax}\left\{r_{k} s_{k} \mid k \in K\right\}$ na solução ótima de $\left(L B_{I J}\right)$.

É preciso algum cuidado para garantir que os novos nós gerados pela escolha de ramificação ainda correspondam a subpartições separáveis. Isso é verdade se $A_{k} \notin \mathcal{R}_{I J} \cup \mathcal{S}_{I J}$, o que pode ser verificado resolvendo-se um ou dois PLs, como visto na seção 1.3. É claro que normalmente apenas um PL será necessário, de acordo com a verificação de $A_{k} x>A_{k} y$ ou $A_{k} x<A_{k} y$ na solução de $\left(L B_{I J}\right)$; no primeiro caso o teste acima se reduz a $A_{k} \notin \mathcal{R}_{I J}$, no segundo se reduz a $A_{k} \notin \mathcal{S}_{I J}$. Na improvável hipótese de valer $A_{k} x=A_{k} y$ na solução de $\left(L B_{I J}\right)$, então poderá ser necessária a. resolução de dois problemas lineares.

Se for verificado que $A_{k} \in \mathcal{R}_{I J}$ (ou $A_{k} \in \mathcal{S}_{I J}$ ) então os conjuntos de índices correspondentes são atualizados, $I \leftarrow I \cup\{k\}$ (ou $J \leftarrow J \cup\{k\}$ respectivamente) e $K \leftarrow K \backslash\{k\}$, e o próximo índice $k=\operatorname{argmax}\left\{r_{k} s_{k} \mid k \in K\right\}$ é escolhido. Se $K$ tornar-se vazio, então $(I, J)$ é uma partição de $\{1,2, \ldots, n\}$; o problema quadrático $\left(\mathrm{HF}_{I J}\right)$ correspondente é resolvido e o nó da árvore é fechado.

Se $k$ é uma escolha de ramificação válida, então dois novos nós são acrescentados à arvore de enumeração, correspondendo aos problemas $\left(\mathrm{HF}_{I^{\prime} J}\right)$ e $\left(\mathrm{HF}_{I J^{\prime}}\right)$, onde $I^{\prime}=I \cup\{k\}$ e $J^{\prime}=J \cup\{k\}$.

\section{Corretude e Complexidade do Método Enumerativo}

A partir das discussão acima, pode-se estabelecer o seguinte resultado. 
Proposição 1.19 (Corretude e complexidade do método Enumerativo) O método apresentado nesta seção é correto, isto é, sempre encontra a solução ótima do problema (HF). Além disto, o número total de nós da árvore de enumeração é limitado por

$$
2 v_{m}(n)-1=2 \sum_{i=0}^{m}\left(\begin{array}{c}
n-1 \\
i
\end{array}\right)-1
$$

Prova : A corretude é conseqüência dos fatos que a solução ótima do problema está associada a alguma partição separável, e que todas as partições separáveis são implicitamente enumeradas pelo método. A limitação acima é conseqüência do teorema 1.6 e do fato que uma árvore binária com $v_{m}(n)$ folhas possui $v_{m}(n)-1$ nós internos.

\subsection{O Problema de Ajuste Linear de Dobradiças}

Nesta seção uma formulação alternativa do problema de Ajuste de Dobradiças é introduzida, que se baseia no uso da norma $l_{1}$ para medição dos erros, ao invés da norma Euclideana. Esta formulação corresponde a um MPEC com função objetivo linear, que pode ser resolvido por métodos mais eficientes. Um destes métodos é o algoritmo SLCP, que será discutido na seqüência.

\section{Observação 1.20 (Normas para medição dos erros)}

Vale a pena lembrar que diferentes normas para medição dos erros em um problema de aproximação definem diferentes objetivos de aproximação. Isto pode ser observado através de um simples exemplo no contexto de regressão linear, como a seguir. 
Considere os três pontos dados abaixo. A solução do problema de regressão linear com a norma Euclideana é indicada pela linha contínua.

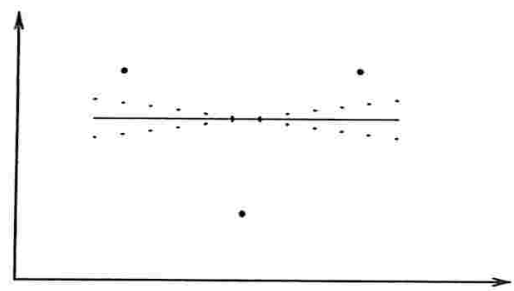

As linhas pontilhadas indicam outras aproximações lineares para os mesmos três pontos, que possuem o mesmo erro em relação à norma $l_{1}$. A solução ótima para o problema de regressão linear com a norma $l_{1}$ será dado pela linha indicada abaixo.

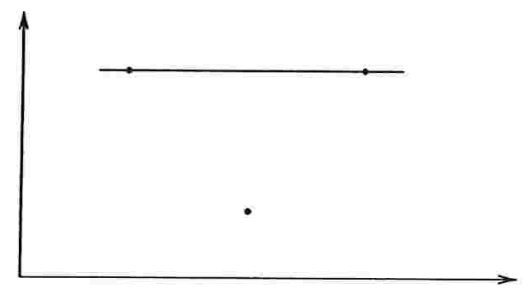

Completando o exemplo, pode-se ver que uma regressão com a norma $l_{\infty}$, por outro lado, tentaria não se afastar muito de nenhum dos pontos dados. A solução ótima para o exemplo acima seria paralela àquela dada pela norma Euclideana, porém na exata metade da distância entre os pontos de cima e o de baixo.

O problema original com a norma Euclideana substituída pela norma $l_{1}$ pode ser formulado como

$$
\min \|\operatorname{vecmax}\{A x, A y\}-b\|_{1} \text {. }
$$

Modelando a diferença vecmax $\{A x, A y\}-b$ como uma variável $\sigma$ e a $\|\cdot\|_{1}$ como uma variável $\gamma$, o problema torna-se 
Definição 1.21 (Problema de Ajuste Linear de Dobradiças - LHF)

$$
(L H F) \begin{cases}\min & \mathbb{1}^{t} \gamma \\ \text { s.a. } & r=\sigma-A x+b \\ & s=\sigma-A y+b \\ & \gamma \geq \sigma \\ & \gamma \geq-\sigma \\ & r, s \geq 0 \\ & r^{t} s=0 .\end{cases}
$$

Uma solução algorítmica possível consiste em adaptar a abordagem enumerativa da seção anterior ao problema de Ajuste Linear de Dobradiças. Como todas as considerações geométricas das seções 1.1 e 1.3 estão relacionadas ao conjunto de pontos viáveis de (HF), e portanto independem da norma Euclideana, a principal diferença é o cômputo de limitantes inferiores e superiores. O cômputo de um limitante inferior para o nó $(I, J)$ da árvore de enumeração pode ser feito resolvendo-se o seguinte problema de programação linear

$$
(L L B)_{I J} \begin{cases}\min & \mathbb{1}^{t} \gamma \\ \text { s.a. } & r=\sigma-A x+b \\ & s=\sigma-A y+b \\ & \gamma \geq \sigma \\ & \gamma \geq-\sigma \\ & r_{I}=0 \\ & s_{J}=0 \\ & r, s \geq 0 .\end{cases}
$$

O Algoritmo para Obtenção de Dobradiças (HFA - seção 1.2) também pode ser adaptado para esta formulação, e pode ser usado no cômputo dos limitantes 
superiores. Os vetores $x^{\varphi}$ e $y^{\varphi}$ no passo principal do algoritmo HFA são definidos respectivamente como as aproximações dadas pela regressão linear de $\left\{\left(A_{i}, b_{i}\right)\right\}_{i \in I}$ e $\left\{\left(A_{j}, b_{j}\right)\right\}_{j \in J}$ usando a norma $l_{1}$. Estas aproximações podem ser calculadas como as soluções dos seguintes problemas de programação linear.

$$
\left\{\begin{array} { l l } 
{ \operatorname { m i n } } & { \mathbb { 1 } ^ { t } \gamma _ { I } } \\
{ \text { s.a. } } & { \gamma _ { I } \geq A _ { I } x - b _ { I } } \\
{ } & { \gamma _ { I } \geq - A _ { I } x + b _ { I } , }
\end{array} \quad \left\{\begin{array}{ll}
\min & \mathbb{1}^{t} \gamma_{J} \\
\text { s.a. } & \gamma_{J} \geq A_{J} x-b_{J} \\
& \gamma_{J} \geq-A_{J} x+b_{J}
\end{array}\right.\right.
$$

Mais detalhes sobre teoria e métodos eficientes de solução para regressão linear com norma $l_{1}$ podem ser encontrados em [Spath 1992].

Os limitantes superiores heurísticos para o método enumerativo também podem ser adaptados, tanto a solução obtida diretamente do limitante inferior, como as soluções de subproblemas associados a partições separáveis, que são também problemas de programação linear.

Uma desvantagem desta abordagem é o fato de que o tamanho dos subproblemas cresce significativamente. Por outro lado, um procedimento eficiente para processar MPECs lineares com restrições dadas por um LCP geral foi proposto em [Júdice \& Faustino 1992]. O LCP geral é

$$
\left\{\begin{array}{l}
w=q+M z+N y \\
z^{t} w=0 \\
z, w, y \geq 0
\end{array}\right.
$$

Para reescrever o (LHF) como um LCP geral, as variáveis livres de sinal $x, y$ e $\sigma$ são substituídas por variáveis não-negativas $u, u_{n+1}, v, v_{n+1}, \alpha$ e $\beta$, utilizando-se as seguintes substituições

$$
\left\{\begin{array}{l}
x=u-u_{n+1} \rrbracket \\
y=v-v_{n+1} \rrbracket \\
\sigma=\alpha-\beta
\end{array}\right.
$$


Mais ainda, impondo a condição de complementaridade $\alpha^{t} \beta=0$ é possível expressar $\left|\sigma_{i}\right|$ como $\alpha_{i}+\beta_{i}$. Então, após reordenar os termos, obtém-se o seguinte problema equivalente:

$$
\begin{cases}\min & \mathbb{1}^{t}(\alpha+\beta) \\ \text { s.a. } & \beta=b+\alpha-r-A u+(A \mathbb{1}) u_{n+1} \\ & \beta+s=b+\alpha-A v+(A \mathbb{1}) v_{n+1} \\ & r^{t} s=\alpha^{t} \beta=0 \\ & r, s, \alpha, \beta, u, u_{n+1}, v, v_{n+1} \geq 0\end{cases}
$$

Substituindo $\beta=b+\alpha-r-A u+(A 1) u_{n+1}$ na segunda equação e na função objetivo, e agrupando as variáveis como $w=\left[\begin{array}{c}\beta \\ s\end{array}\right], z=\left[\begin{array}{c}\alpha \\ r\end{array}\right]$ e $y=\left[\begin{array}{c}u \\ u_{n+1} \\ v \\ v_{n+1}\end{array}\right]$, o problema torna-se

$$
(\mathrm{LHF}) \begin{cases}\min & \mathbb{1}^{t} b+c^{t} z+d^{t} y \\ \text { s.a. } & w=q+M z+N y \\ & z, w, y \geq 0 \\ & z^{t} w=0\end{cases}
$$

onde $c=\left[\begin{array}{c}2 \rrbracket \\ -1\end{array}\right], d=\left[\begin{array}{c}-A^{t} \rrbracket \\ c^{t} \rrbracket \\ 0 \\ 0\end{array}\right], q=\left[\begin{array}{l}b \\ 0\end{array}\right], M=\left[\begin{array}{cc}I & -I \\ 0 & I\end{array}\right]$ e $N=$ $\left[\begin{array}{cccc}-A & A \rrbracket & 0 & 0 \\ A & -A \rrbracket & -A & A \rrbracket\end{array}\right]$. 
O algoritmo do Problema de Complementaridade Linear Seqüêncial (SLCP) para encontrar o mínimo global do problema de Ajuste Linear de Dobradiças é delineado a seguir. Os detalhes do algoritmo podem ser encontrados em [Júdice \& Faustino 1992].

O algoritmo SLCP resolve uma seqüência de LCPs gerais obtidos a partir do MPEC linear de modo a garantir o decréscimo monótono do valor da função objetivo. O subproblema resolvido a cada passo é parametrizado por $\lambda$, como abaixo.

$$
\left(\mathrm{LHF}_{\lambda}\right)\left\{\begin{array}{l}
v_{0}=\lambda-\mathbb{1}^{t} b-c^{t} z-d^{t} y \\
w=q+M z+N y \\
z, w, y, v_{0} \geq 0 \\
z^{t} w=0
\end{array}\right.
$$

O algoritmo é apresentado a seguir. A precisão relativa da solução depende de um parâmetro $\gamma>0$ dado.

\section{O Algoritmo SLCP para o Ajuste Linear de Dobradiças}

0. Seja $\gamma$ um número positivo pequeno. Resolva o LCP geral correspondente ao conjunto de pontos viáveis de (LHF) e seja $\left(z^{*}, w^{*}, y^{*}\right)$ a solução obtida. Faça $U B \longleftarrow \mathbb{1}^{t} b+c^{t} z^{*}+d^{t} y^{*}$.

1. Seja $\lambda=U B-\gamma|U B|$, e resolva o problema $\left(\operatorname{LHF}_{\lambda}\right)$. Se este problema não tiver solução, pare! Caso contrário, seja $\left(z^{*}, w^{*}, y^{*}\right)$ a solução obtida.

2. Faça $U B \longleftarrow \mathbb{1}^{t} b+c^{t} z^{*}+d^{t} y^{*}$ e volte ao passo 1 .

O último passo do algoritmo consiste em provar que o subproblema $\left(\mathrm{LHF}_{\lambda}\right)$ é inviável, com $\lambda$ minimamente abaixo do valor ótimo do MPEC original. Este é um 
problema muito difícil, e é discutido em detalhe em [Júdice \& Faustino 1992].

$\mathrm{O}$ critério de parada correspondente à inviabilidade do subproblema $\left(\mathrm{LHF}_{\lambda}\right)$ leva imediatamente à conclusão de que o último limitante superior obtido é um valor $\varepsilon$-otimal para o problema (LHF), onde $\varepsilon$ é dado por

$$
\varepsilon=\gamma\left|\mathbb{1}^{t} b+c^{t} z^{*}+d^{t} y^{*}\right|
$$

É também interessante notar que a matriz $M$ no subproblema $\left(\operatorname{LHF}_{\lambda}\right)$ é positiva definida, pois para quaisquer $x$ e $y$,

$$
\left[\begin{array}{l}
x \\
y
\end{array}\right]^{t}\left[\begin{array}{cc}
I & -I \\
0 & I
\end{array}\right]\left[\begin{array}{l}
x \\
y
\end{array}\right]=\frac{1}{2}\left[x^{t} x+y^{t} y+(x-y)^{t}(x-y)\right],
$$

que é sempre não-negativo, e é igual a zero se, e somente se, $x=y=0$.

A eficiência do método é diretamente relacionada à eficiência do procedimento usado para resolver os subproblemas $\left(\mathrm{LHF}_{\lambda}\right)$. Os métodos altamente eficientes disponíveis para a resolução de LCPs com matrizes positivas definidas [Cottle, Pang \& Stone 1992] não podem ser aplicados ao LCP geral. Estes subproblemas são portanto resolvidos por um método enumerativo híbrido [Júdice \& Faustino 1992], que não impõe condições às classes de matrizes envolvidas na definição do LCP geral.

\subsection{Experiência Computacional}

Todos os algoritmos descritos anteriormente foram implementados em C. Eles foram aplicados a 10 problemas-teste de médio porte gerados aleatoriamente, em um Pentium III 1.2 GHz rodando Linux 2.2.19.

Para efeito de comparação, os mesmos dados foram submetidos ao algoritmo HFA amortecido de Pucar, Sjöberg \& Breiman, aos métodos Enumerativos das seções 1.3 e 1.4, e ao algoritmo SLCP de Júdice \& Faustino. 
O algoritmo HFA amortecido é usado como inicialização do método Enumerativo, portanto não se pretende comparar o desempenho destes métodos. Eles são utilizados para mostrar valores iniciais (mínimos locais) típicos, em oposição aos valores ótimos destes problemas não-convexos. Para inicializar o HFA amortecido, uma partição balanceada é utilizada como entrada, para tentar evitar a convergência para a partição trivial. O valor do quadrado do erro da aproximação da solução do HFA é dado, bem como o número de iterações e o tempo de execução.

Os subproblemas lineares e quadráticos no método Enumerativo são resolvidos através do Algoritmo de Pivotação Complementar de Lemke. O limitante superior inicial (passo 0) é a solução do HFA. O limitante superior computado no passo 4 é a solução do HFA usando como ponto inicial a solução do problema relaxado naquele nó. Não são fixadas variáveis adicionais no passo 2. Estas opções foram escolhidas como aquelas que minimizam (empiricamente) o tempo de execução para os testes apresentados, e são as configurações padrão.

A fim de manter pequeno o tamanho dos subproblemas LCP, as variáveis de folga $r$ e $s$ são eliminadas, e as substituições $\sigma_{I}=A_{I} x-b_{I}$ e $\sigma_{J}=A_{J} y-b_{J}$ são consideradas. O problema relaxado para limitação inferior torna-se

$$
(L B)_{I J} \begin{cases}\min & \left(A_{I} x-b_{I}\right)^{t}\left(A_{I} x-b_{I}\right)+\left(A_{J} y-b_{J}\right)^{t}\left(A_{J} y-b_{J}\right)+\sigma_{K}^{t} \sigma_{K} \\ \text { s.a. } \quad & A_{I} x-A_{I} y \geq 0 \\ & -A_{J} x+A_{J} y \geq 0 \\ & \sigma_{K} \geq A_{K} x-b_{K} \\ & \sigma_{K} \geq A_{K} y-b_{K} .\end{cases}
$$

A tabela abaixo resume a experiência computacional para o problema de Ajuste Euclideano de Dobradiças. As dimensões da formulação como MPEC dos problemas (HF) são dadas; o número total de variáveis é portanto $3 n+2 m$, o número de pares de variáveis complementares é $n$ e o número de restrições lineares é $2 n$. 
As medidas de desempenho do método Enumerativo são o número de problemas quadráticos resolvidos (que é igual ao tamanho da árvore de enumeração), o número de problemas lineares, o tempo de execução e o valor ótimo. Para evitar testes demasiadamente longos, um número limite de nós foi estabelecido para o tamanho da árvore de enumeração. Nos testes abaixo, a execução era interrompida após a enumeração de 1000 nós. Os testes interrompidos são marcados com um asterisco.

\begin{tabular}{|c|c|c|c|c|c|c|c|c|c|c|c|c|}
\hline & \multicolumn{4}{|c|}{ Testes } & \multicolumn{3}{|c|}{ Sol. Inicial (HFA) } & \multicolumn{5}{|c|}{ Sol. Final (Enumerativo) } \\
\hline $\begin{array}{l}\text { \# } \\
0 \\
\stackrel{\omega}{W} \\
\stackrel{\omega}{\omega}\end{array}$ & 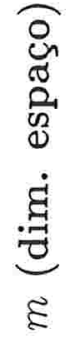 & 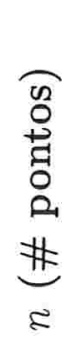 & 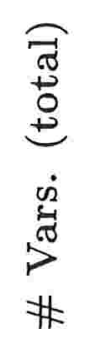 & 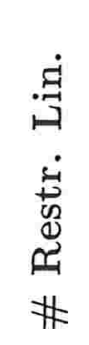 & $\begin{array}{l}\dot{D} \\
\dot{0} \\
\stackrel{0}{ \pm} \\
\# \\
\#\end{array}$ & $\begin{array}{l}\overparen{0} \\
\circ \\
\stackrel{0}{0} \\
\text { वृ } \\
\ddot{\oplus}\end{array}$ & $\frac{\dot{0}}{\frac{\pi}{p}}$ & $\begin{array}{l}\stackrel{n}{O} \\
A_{1} \\
\#\end{array}$ & $\begin{array}{l}\stackrel{\infty}{-} \\
\text { \# }\end{array}$ & 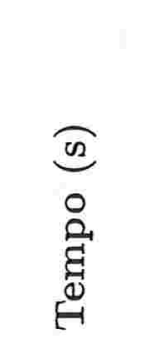 & 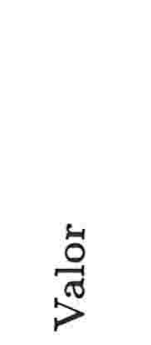 & 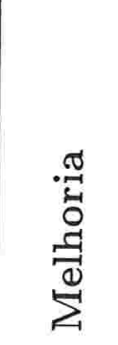 \\
\hline 1 & 5 & 30 & 100 & 60 & 20 & 0.01 & 1.3028 & 84 & 45 & 3.13 & 1.2023 & 1.08 \\
\hline 2 & 10 & 35 & 125 & 70 & 11 & 0.01 & 0.9905 & 354 & 178 & 27.80 & 0.5306 & 1.87 \\
\hline $3^{*}$ & 15 & 40 & 150 & 80 & 19 & 0.02 & 0.6212 & 1000 & 655 & 167.25 & 0.1256 & 4.94 \\
\hline $4^{*}$ & 20 & 45 & 175 & 90 & 30 & 0.06 & 0.5404 & 1000 & 911 & 296.96 & 0.0166 & 32.40 \\
\hline 5 & 25 & 50 & 200 & 100 & 16 & 0.06 & 2.5227 & 26 & 27 & 11.61 & 0 & $\infty$ \\
\hline 6 & 30 & 55 & 225 & 110 & 16 & 0.09 & 3.7060 & 56 & 57 & 53.39 & 0 & $\infty$ \\
\hline 7 & 35 & 60 & 250 & 120 & 29 & 0.23 & 4.9130 & 51 & 52 & 56.16 & 0 & $\infty$ \\
\hline 8 & 40 & 65 & 275 & 130 & 59 & 0.62 & 0.2089 & 19 & 20 & 25.73 & 0 & $\infty$ \\
\hline 9 & 45 & 70 & 300 & 140 & 41 & 0.58 & 0.3080 & 106 & 107 & 231.01 & 0 & $\infty$ \\
\hline 10 & 50 & 75 & 325 & 150 & 73 & 1.36 & 0.2446 & 105 & 106 & 284.93 & 0 & $\infty$ \\
\hline
\end{tabular}

A última coluna da tabela mostra a melhoria na qualidade da solução do método Enumerativo com respeito à solução do método HFA amortecido. Esta melhoria é calculada como

$$
\frac{\text { Valor HFA }}{\text { Valor Enumerativo }} \text {. }
$$


Os valores infinitos nesta coluna correspondem a ajustamentos perfeitos que não foram obtidos pelo algoritmo HFA. Nestes casos observa-se que o número de nós enumerados é surpreendentemente pequeno. Isso se deve ao fato de que 0 é um limitante inferior natural para o problema original, portanto no momento em que uma solução com valor 0 é detectada, o algoritmo pára. Se, além disso, os dados de entrada correspondem a uma dobradiça, então o algoritmo pode ser acelerado ao se notar que qualquer nó $(I, J)$ com limitante inferior LB maior do que 0 pode ser podado.

O esforço relativo do método Enumerativo com respeito à enumeração completa das partições separáveis é uma medida de quão efetivo é o processo de poda. Esta relação pode ser calculada para cada teste como

$$
\frac{\# \text { QPs }}{v_{m}(n)}
$$

onde $v_{m}(n)$ é dado pelo teorema 1.6. Para os testes acima, esta medida começa com os valores $0.003017,0.000005$ e a partir do teste número 3 ela é identicamente 0. A partir destes testes poder-se-ia conjecturar que esta relação tende a 0 quando $m, n \longrightarrow \infty$, e isto significaria que o tamanho da árvore de enumeração é na realidade $o\left(v_{m}(n)\right)$.

Os mesmos 10 problemas de médio porte foram reformulados como problemas de Ajuste Linear de Dobradiças e resolvidos pelo método Enumerativo adaptado, bem como pelo algoritmo SLCP aplicado à formulação como MPEC linear (seção 1.4).

O subproblema de limitação inferior para o Ajuste Linear de Dobradiças torna-se 


$$
(L L B)_{I J} \begin{cases}\min & \mathbb{1}^{t} \gamma \\ \text { s.a. } & A_{I} x-A_{I} y \geq 0 \\ & -A_{J} x+A_{J} y \geq 0 \\ & \sigma_{K} \geq A_{K} x-b_{K} \\ & \sigma_{K} \geq A_{K} y-b_{K} \\ & \gamma_{I} \geq A_{I} x-b_{I} \\ & \gamma_{I} \geq-A_{I} x+b_{I} \\ & \gamma_{J} \geq A_{J} y-b_{J} \\ & \gamma_{J} \geq-A_{J} y+b_{J} \\ & \gamma_{K} \geq \sigma_{K} \\ & \gamma_{K} \geq-\sigma_{K} .\end{cases}
$$

O tamanho das árvores de enumeração do método Enumerativo adaptado são dadas, bem como o número de PLs auxiliares resolvidos em cada passo. As medidas para o algoritmo SLCP são o número de iterações e o tempo de execução. Como o último LCP geral resolvido é sempre inviável, qualquer método enumerativo tem que explorar toda a árvore de enumeração para detectar esta propriedade. A fim de comparar o esforço relativo do SLCP até encontrar a solução ótima com o esforço necessário para provar que o último subproblema é inviável, a tabela apresenta tanto o tempo de execução até que a solução seja encontrada (marcado como "Tempo Sol." na tabela) quanto o tempo de execução total. 


\begin{tabular}{|c|c|c|c|c|c|c|c|c|c|c|}
\hline \multicolumn{3}{|c|}{ Testes } & \multicolumn{4}{|c|}{ Enumerativo } & \multicolumn{4}{|c|}{ SLCP } \\
\hline 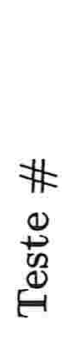 & 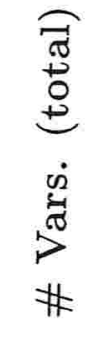 & 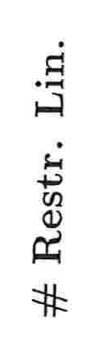 & $\begin{array}{l}0 \\
\text { 告 } \\
\text { Z } \\
\#\end{array}$ & $\begin{array}{l}\stackrel{n}{1} \\
\# \\
\#\end{array}$ & 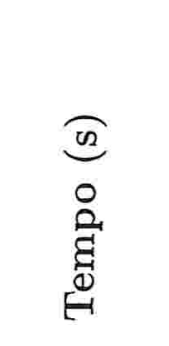 & $\frac{\ddot{0}}{p}$ & 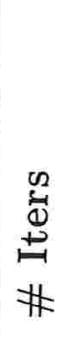 & 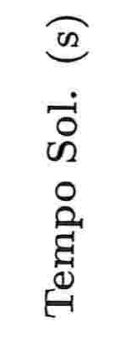 & \begin{tabular}{l} 
(n) \\
$\circ$ \\
0 \\
$\stackrel{0}{0}$ \\
\multirow{0}{0}{}
\end{tabular} & 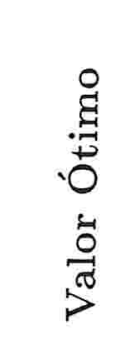 \\
\hline 1 & 132 & 60 & 412 & 328 & 222.79 & 4.3626 & 6 & 0.23 & 7.49 & 4.3626 \\
\hline $2^{*}$ & 162 & 70 & 1000 & 507 & 1419.68 & 2.6287 & 10 & 6.62 & 21.47 & 2.6287 \\
\hline $3^{*}$ & 192 & 80 & 1000 & 504 & 2776.37 & 1.4140 & 21 & 0.59 & 21.85 & 1.1230 \\
\hline $4^{*}$ & 222 & 90 & 1000 & 505 & 5039.91 & 0.3051 & 17 & 16.10 & 31.69 & 0.1456 \\
\hline 5 & 252 & 100 & 691 & 354 & 5333.28 & 0 & 8 & 0.43 & 0.43 & 0 \\
\hline 6 & 282 & 110 & 58 & 36 & 638.27 & 0 & 11 & 0.22 & 0.22 & 0 \\
\hline 7 & 312 & 120 & 51 & 33 & 753.91 & 0 & 5 & 2.12 & 2.12 & 0 \\
\hline 8 & 342 & 130 & 56 & 38 & 1157.89 & 0 & 4 & 2.80 & 2.80 & 0 \\
\hline 9 & 372 & 140 & 56 & 39 & 1509.92 & 0 & 1 & 3.76 & 3.76 & 0 \\
\hline 10 & 402 & 150 & 57 & 43 & 2410.00 & 0 & 1 & 4.19 & 4.19 & 0 \\
\hline
\end{tabular}

Note que, dos três testes interrompidos pelo algoritmo Enumerativo adaptado por atingirem o número limite de nós, dois não atingiram o valor ótimo dado pela solução do SLCP.

O desempenho do método Enumerativo para o Ajuste Linear de Dobradiças é prejudicado por duas razões principais: o tamanho dos subproblemas, e o número de nós que se torna necessário explorar. O tamanho da árvore de enumeração depende da qualidade dos limitantes inferiores e superiores. Aparentemente, o limitante inferior dado pela relaxação e o limitante superior obtido pelo HFA adaptado não são tão justos no caso linear quanto no caso Euclideano. Além disso, o HFA adaptado resolve dois PLs a cada passo, e é portanto computacionalmente mais caro do que o HFA 
original, que resolve dois problemas de regressão linear, ou seja, dois sistemas lineares, a cada passo.

Por outro lado, o algoritmo SLCP teve um bom desempenho, levando menos de 10 segundos para encontrar a solução na maioria dos testes. O número total de iterações, que corresponde ao número de LCPs gerais resolvidos, também é baixo.

Os problemas de Ajuste Linear e Euclideano de Dobradiças têm as mesmas entradas, e diferem nos tipos de aproximações obtidas. Se, numa aplicação prática, a escolha da norma para medição dos erros não é pré-determinada pelo modelo específico, então a escolha da norma $l_{1}$ leva a um problema MPEC mais simples, e a solução algorítmica via SLCP é recomendada.

\subsection{Conclusão e Trabalho Futuro}

Este capítulo apresentou um algoritmo enumerativo para a otimização global do problema de Ajuste de Dobradiças. Seus detalhes de implementação e testes computacionais foram apresentados, mostrando que ele é adequado para aproximar funções não-lineares por uma função dobradiça, que é o subproblema fundamental de ajustamento de funções não-lineares dentro do modelo de aproximação HH.

A reformulação do problema com a norma $l_{1}$ como um MPEC linear foi apresentada, assim como um método eficiente de solução através da abordagem do Seqüencial LCP.

Uma das questões em aberto com relação a este trabalho é se o algoritmo SLCP pode tornar-se mais eficiente quando a matriz $M$ no problema MPEC linear é positiva definida. Matrizes positivas definidas têm um papel fundamental em problemas de complementaridade. Por exemplo, o LCP clássico com uma matriz positiva definida é equivalente a um problema de programação quadrática estritamente convexo e pode ser resolvido muito eficientemente. Os subproblemas resolvidos pelo 
algoritmo SLCP são na realidade LCPs gerais, mas a propriedade de matrizes positivas definidas na parte complementar dos subproblemas poderia ser usada para acelerar a sua resolução. 


\section{Capítulo 2}

\section{O Problema de Valores Próprios Complementar Simétrico}

O cálculo de valores próprios é de importância crucial em vários problemas práticos em física e engenharia. Valores Próprios estão relacionados com freqüências de ressonância de estruturas, e com a estabilidade de sistemas dinâmicos [Chatelin 1993]. Uma parcela expressiva destes problemas práticos envolve matrizes reais simétricas [Parlett 1997].

Uma abordagem clássica para resolver problemas de valores próprio com matrizes reais simétricas de grande porte é aplicar técnicas de otimização irrestrita à função conhecida como quociente de Rayleigh, que corresponde ao valor da forma quadrática induzida pela matriz, calculada no bordo da esfera unitária. Esta função tem a propriedade que todo ponto de equilíbrio está sobre um vetor próprio, com o correspondente valor próprio dado pelo valor do quociente de Rayleigh.

O Problema de Valores Próprios Complementar (EiCP) surgiu no estudo de estados de equilíbrio estático de sistemas mecânicos com atrito unilateral [Júdice, Costa, Figueiredo \& Martins 2000]. O tratamento rigoroso do problema mo- 
tivador depende de uma ampla base de conhecimentos em Mecânica, e foge ao escopo do presente trabalho. A seguir é apresentada uma visão panorâmica da modelagem daquele problema, cuja única finalidade é dar a conhecer uma possível aplicação prática dos resultados aqui apresentados.

Um sistema elástico linear de dimensão finita é considerado, sujeito a forças planares constantes, com atrito de contato com um obstáculo achatado fixo. O deslocamento $u(t)$ e as reações $r(t)$ satisfazem as condições de contato unilateral de Signorini

$$
u_{N}(t) \leq 0, r_{N}(t) \leq 0, u_{N}(t)^{t} r_{N}(t)=0
$$

(note a complementaridade), e a lei de atrito de Coulomb

$$
\left\|r_{T}(t)\right\|+\mu r_{N}(t) \leq 0,\left\|\dot{u}_{T}(t)\right\| r_{T}(t)-\mu \dot{u}_{T}(t) r_{N}(t)=0
$$

onde $\mu>0$ é o coeficiente de atrito, $N$ e $T$ são conjuntos de índices das componentes normal e tangencial, e () indica a derivada com respeito a $t$. Sob a aplicação de forças $f^{0}$ constantes, a evolução dinâmica do sistema é governada pelas equações de equilíbrio de momento

$$
M \ddot{u}(t)+K u(t)=f^{0}+r(t)
$$

onde $M$ e $K$ são matrizes simétricas positivas definidas representando massa e rigidez, respectivamente. Um estado de equilíbrio do sistema acima é caracterizado por um vetor de deslocamento $u^{0}$ e um vetor de reação $r^{0}$ que satisfazem o sistema de equilíbrio

$$
\left\{\begin{array}{l}
K u^{0}=f^{0}+r^{0} \\
u_{N}^{0} \leq 0 \\
r_{N}^{0} \leq 0 \\
\left(u_{N}^{0}\right)^{t} r_{N}^{0}=0 \\
\left\|r_{T}^{0}\right\|+\mu r_{N}^{0} \leq 0 .
\end{array}\right.
$$


Pode-se mostrar [Costa, Figueiredo, Júdice \& Martins 2001] que, para alguma vizinhança $[\tau, \tau+\Delta \tau)$, existem soluções dinâmicas da forma

$$
\begin{aligned}
& u(t)=u^{0}+\alpha(t) v \\
& v(t)=v^{0}+\beta(t) w
\end{aligned}
$$

(onde $v$ e $w$ são taxas de deslocamento e de reação constantes) se, e somente se, existe uma solução do sistema abaixo, $\operatorname{com} \lambda>0$ e $v \neq 0$,

$$
\left\{\begin{array}{l}
w=\left(\lambda^{2} M^{*}+K^{*}\right) v \\
w_{F}=0, v_{N}=0, v_{D}=0 \\
v_{T} \operatorname{sinal}\left(r_{T}^{0}\right) \leq 0 \\
w_{T} \operatorname{sinal}\left(r_{T}^{0}\right)+\mu w_{N} \leq 0 \\
\left(v_{T} \operatorname{sinal}\left(r_{T}^{0}\right)\right)\left(w_{T} \operatorname{sinal}\left(r_{T}^{0}\right)+\mu w_{N}\right)=0
\end{array}\right.
$$

onde $\operatorname{sinal}\left(r_{T}^{0}\right)$ é o vetor com componentes \pm 1 de acordo com o sinal de $r_{T}^{0}, F$ são partículas sem contato, $D$ são partículas com taxa de deslocamento zero, e $M^{*}$ e $K^{*}$ são deslocamentos lineares das matrizes $M$ e $K$, respectivamente. Este problema pode ser reformulado como um Problema de Valores Próprios Complementar Geral,

$$
(\mathrm{GEiCP})_{J}: \text { Encontre } \lambda>0, x \neq 0 \text { tal que }\left\{\begin{array}{l}
w=(\lambda B-A) x \\
w_{\bar{J}}=0 \\
w_{J} \geq 0 \\
x_{J} \geq 0 \\
w_{J}^{t} x_{J}=0
\end{array}\right.
$$

que é um dos problemas aqui considerado. Maiores detalhes podem ser encontrados em [Costa, Figueiredo, Júdice \& Martins 2001].

O objetivo deste capítulo é estudar o (EiCP) com matrizes $A$ e $B$ simétricas. Prova-se que este problema em particular é equivalente ao problema de encontrar um ponto de equilíbrio do quociente de Rayleigh generalizado, sujeito às restrições 
adicionais $x \geq 0$ e $\lambda>0$, que pode ser resolvido usando qualquer método de subida de gradiente, a partir de um ponto inicial conveniente. Este resultado é similar a um teorema clássico para problemas de complementaridade não-lineares (NCP), que não poderia ser aplicado à reformulação do (EiCP) como NCP, como será visto na seção 2.3. O resultado estende-se ao Problema de Valores Próprios Complementar Geral (GEiCP) simétrico, que é uma generalização do (EiCP) simétrico de grande interesse prático. Testes computacionais mostram que esta reformulação permite a resolução dos problemas simétricos em menor tempo e com maior robustez (certeza de resolução). Alguns resultados preliminares da pesquisa com o caso assimétrico são também apresentados.

A estrutura deste capítulo é a seguinte.

Na seção 2.1 é discutido o problema clássico de valores próprios, com ênfase para o caso de matrizes simétricas. A relação deste problema com a otimização do quociente de Rayleigh é estabelecida, mostrando que os valores próprios deste problema podem ser computados com métodos de gradiente.

Na seção 2.2 são apresentados o Problema de Valores Próprios Complementar e o Problema de Valores Próprios Complementar Geral. São apresentados alguns resultados gerais acerca da viabilidade destes problemas e do número de soluções dos mesmos. Mostra-se que em geral estes problemas são NP-difíceis. Neste capítulo o termo viabilidade é usado como sinônimo para a existência de solução, o que significa a existência de uma solução complementar nos casos do (EiCP) e (GEiCP).

O caso simétrico é estudado na seção 2.3, onde mostra-se que tanto o (EiCP) quando o (GEiCP) simétricos são equivalentes a encontrar pontos de equilíbrio de problemas de otimização, e são portanto de solução relativamente fácil. Os testes computacionais são apresentados na seção 2.4 .

A seção 2.5 apresenta algumas conclusões e trabalho futuro, e alguns resultados preliminares no caso assimétrico são apresentados no apêndice. 


\subsection{Problemas de Valores Próprios e Otimização}

O problema clássico de valores próprios (EiP) é definido a seguir.

Definição 2.1 (Problema Clássico de Valores Próprios - EiP)

Dada uma matriz $A \in \mathbb{R}^{n \times n}$,

(EiP): Encontre $\lambda \in \mathbb{R}, x \neq 0$ tal que $A x=\lambda x$.

Uma condição necessária e suficiente para a existência de uma solução com $x \neq 0$ é que o espaço nulo da matriz $(A-\lambda I)$ seja não-trivial, e isso acontece se, e somente se, $\lambda$ é raiz do polinômio $\operatorname{det}(A-\lambda I)=0$. Tais valores de $\lambda$ são chamados de valores próprios de $A$, e são as soluções do (EiP). Como o polinômio $\operatorname{det}(A-\lambda I)$ tem grau $n$, o (EiP) possui no máximo $n$ soluções distintas.

Se $\bar{\lambda}$ é um valor próprio de $A$, encontrar um vetor próprio correspondente resume-se a encontrar uma solução não-trivial do sistema linear $(A-\bar{\lambda} I) x=0$. Como este sistema é homogêneo, $x$ é um vetor próprio associado a $\bar{\lambda}$ se, e somente se, $\alpha x$ também é um vetor próprio associado a $\bar{\lambda}$, para qualquer $\alpha \neq 0$. Um tratamento extensivo das propriedades e algoritmos para o (EiP) pode ser encontrado em [Parlett 1997] e [Chatelin 1993].

Uma das abordagens clássicas para resolver problemas (EiP) de grande porte com matrizes reais simétricas consiste em aplicar técnicas de otimização irrestrita ao quociente de Rayleigh, definido a seguir. 
Definição 2.2 (Quociente de Rayleigh)

O quociente de Rayleigh é a função

$$
\lambda(x)=\frac{x^{t} A x}{x^{t} x}
$$

definida para todo $x \neq 0$.

\section{Lema 2.3 ((EiP) como problema de otimização)}

$x \neq 0$ é solução do (EiP) se, e somente se, $x$ é um ponto de equilíbrio do quociente de Rayleigh.

Prova : Note que o gradiente do quociente de Rayleigh é

$$
\nabla \lambda(x)=\frac{2}{x^{t} x}[A-\lambda(x) I] x .
$$

Como $\nabla \lambda(x)=0$ se, e somente se, $[A-\lambda(x) I] x=0$, qualquer ponto de equilíbrio $(x, \lambda(x))$ do quociente de Rayleigh é solução do (EiP), e viceversa.

\section{Observação 2.4 (Importância da simetria)}

Note que a expressão do gradiente do quociente de Rayleigh calculado acima só é válida se a matriz $A$ é simétrica. Se $A \neq A^{t}$, a expressão correta é

$$
\nabla \lambda(x)=\frac{1}{x^{t} x}\left[A+A^{t}-2 \lambda(x) I\right] x
$$

e perde-se a relação entre pontos de equilibrio de $\lambda(\cdot)$ e soluções do (EiP).

O Problema de Valores Próprios Geral (GEiP), definido a seguir, recebe atenção freqüente na literatura. 
Definição 2.5 (Problema de Valores Próprios Geral - GEiP)

Dadas $A, B \in \mathbb{R}^{n \times n}$,

(GEiP): Encontre $\lambda \in \mathbb{R}, x \neq 0$ tal que $A x=\lambda B x$.

As soluções do (GEiP) são chamados de valores próprios gerais de $(A, B)$. O (GEiP) é em geral mais difícil que o (EiP) [Parlett 1997]. No caso especial em que $B$ é simétrica positiva definida e $A$ é simétrica, pode-se definir o quociente de Rayleigh generalizado como

Definição 2.6 (Quociente de Rayleigh generalizado)

$O$ quociente de Rayleigh generalizado associado a $A, B$ simétricas, $B$ positiva definida, é a função

$$
\lambda(x)=\frac{x^{t} A x}{x^{t} B x}
$$

definida para todo $x \neq 0$.

\section{Lema 2.7 ((GEiP) como problema de otimização)}

$x \neq 0$ é solução do (GEiP) se, e somente se, $x$ é um ponto de equilibrio do quociente de Rayleigh generalizado.

Prova : O gradiente neste caso é

$$
\nabla \lambda(x)=\frac{2}{x^{t} B x}[A-\lambda(x) B] x
$$

e portanto os pontos de equilíbrio de $\lambda(x)$ correspondem aos vetores e valores próprios gerais de $(A, B)$.

O lema seguinte resume duas propriedades fundamentais do quociente de Rayleigh. 
Lema 2.8 (Homogeneidade de $\lambda(\cdot)$ e ortogonalidade de $\nabla \lambda(\cdot)$ )

Para todo $x \neq 0$ valem as seguintes propriedades:

1. $\lambda(\alpha x)=\lambda(x), \forall \alpha>0$;

2. $x^{t} \nabla \lambda(x)=0$.

\section{Prova :}

1. $\lambda(\alpha x)=\frac{(\alpha x)^{t} A(\alpha x)}{(\alpha x)^{t} B(\alpha x)}=\frac{\alpha^{2} x^{t} A x}{\alpha^{2} x^{t} B x}=\lambda(x)$.

2. $x^{t} \nabla \lambda(x)=x^{t}\left[\frac{2}{x^{t} B x}[A-\lambda(x) B] x\right]=\frac{2}{x^{t} B x}\left[x^{t} A x-\lambda(x) x^{t} B x\right]=$ $\frac{2}{x^{t} B x}\left[x^{t} A x-x^{t} A x\right]=0$.

Como o caso $B$ simétrica positiva definida e $B=I$ são passíveis da mesma abordagem em relação a otimização do quociente de Rayleigh, todos os problemas complementares introduzidos na seção seguinte são formulados com duas matrizes $(A, B)$.

\section{Observação 2.9 (Alternativas ao quociente de Rayleigh)}

Outras funções, cujos pontos de equilibrio correspondem a pares de valores e vetores próprios, existem para classes de matrizes específicas. Por exemplo, as funções $S_{A}(x)=x^{t} x-2 \sqrt{x^{t} A x}, P_{A}(x)=\left(x^{t} x\right)^{2}-2 x^{t} A x$ e $L_{A}(x)=x^{t} x-\ln \left(x^{t} A x\right)$, para uma matriz A simétrica positiva definida, têm seus pontos de equilibrio associados a vetores próprios de $A$, sendo que $S_{A}(\cdot)$ e $P_{A}(\cdot)$ também estão em equilibrio na origem. Os trabalhos de [Mongeau 6 Torki 1999], [Auchmuty 1989] e [Auchmuty 1991] apresentam resultados teóricos e experiências computacionais comparando estas funções ao quociente de Rayleigh para matrizes simétricas positivas definidas. Estas funções não serão abordadas para não restringir o contexto deste trabalho. 


\subsection{O Problema de Valores Próprios Complementar}

Como mencionado anteriormente, o Problema de Valores Próprios Complementar (EiCP) surgiu no estudo de estados de equilíbrio estático de sistemas mecânicos com atrito unilateral [Júdice, Costa, Figueiredo \& Martins 2000]. Este problema pode ser expresso como

Definição 2.10 (Problema de Valores Próprios Complementar - EiCP) Dadas duas matrizes $A, B \in \mathbb{R}^{n \times n}$, com $B$ positiva definida,

$$
\text { (EiCP): Encontre } \lambda>0, x \neq 0 \text { tal que }\left\{\begin{array}{l}
w=(\lambda B-A) x \\
w \geq 0 \\
x \geq 0 \\
w^{t} x=0 .
\end{array}\right.
$$

Note que qualquer solução com $w=0$ corresponde a uma solução do (GEiP). Ao permitir que $w_{J} \geq 0$ sempre que $x_{J}=0$, o (EiCP) possui um conjunto de soluções maior do que o (GEiP), e neste sentido é considerado uma relaxação daquele problema.

O Problema de Valores Próprios Complementar Geral (GEiCP) $J$ é definido a seguir. 


\section{Definição 2.11 (Problema de Valores Próprios Complementar Geral)}

Dadas $A, B \in \mathbb{R}^{n \times n}$, com $B$ definida positiva, $e$ um conjunto de índices $J \subseteq\{1,2, \ldots, n\}, \operatorname{com} \bar{J}=\{1,2, \ldots, n\} \backslash J$,

$$
(G E i C P)_{J}: \text { Encontre } \lambda>0, x \neq 0 \text { tal que }\left\{\begin{array}{l}
w=(\lambda B-A) x \\
w_{\bar{J}}=0 \\
w_{J} \geq 0 \\
x_{J} \geq 0 \\
w_{J}^{t} x_{J}=0
\end{array}\right.
$$

$\mathrm{O}(\mathrm{EiCP})$ é claramente o caso particular do $(\mathrm{GEiCP})_{J}$, com $J=\{1,2, \ldots, n\}$.

Para qualquer solução $(\lambda, x, w)$, o valor $\lambda$ é chamado de valor próprio complementar (geral) de $(A, B)$, e $x$ é um vetor próprio complementar (geral) associado. Como o conjunto de vetores próprios complementares do $(\mathrm{GEiCP})_{J}$ é um cone, não há qualquer perda de generalidade em restringir o problema a encontrar soluções que satisfaçam $\|x\|_{2}=1$. Esta restrição dispensa a restrição $x \neq 0$ que aparece na formulação do problema. No caso do (EiCP) pode-se considerar a restrição linear $\|x\|_{1}=\mathbb{1}^{t} x=1$ ao invés de $\|x\|_{2}=1$, pois $x \geq 0$.

É fácil ver que qualquer solução do (EiCP) ou (GEiCP) com $w=0$ é um valor próprio geral positivo de $(A, B)$ e $x$ é um vetor próprio associado, que satisfaz algumas restrições de sinal. Em geral, vale a seguinte propriedade:

Proposição 2.12 (Conjunto de índices associado a uma solução)

Para qualquer solução $(\lambda, x)$ do $(G E i C P)_{J}$, existe um conjunto I que satisfaz $\bar{J} \subseteq I \subseteq\{1,2, \ldots, n\}$, tal que $\lambda$ é um valor próprio geral positivo de $\left(A_{I I}, B_{I I}\right)$ e $x_{I}$ é um vetor próprio associado, que satisfaz $x_{J \cap I} \geq 0$. 
Prova: Seja $I=\left\{i \mid w_{i}=0\right\}$. Então $I \supseteq \bar{J}$, e $x_{\bar{I}}=0$, por complementaridade. Portanto $A_{I I} x_{I}=\lambda B_{I I} x_{I}$.

No caso do (EiCP), este resultado diz que, dada uma solução $(\lambda, x), \lambda$ é um valor próprio geral positivo de $\left(A_{I I}, B_{I I}\right)$ e $x_{I}$ é um vetor próprio não-negativo associado. Um corolário direto da proposição anterior é que os conjuntos de soluções do (EiCP) e (GEiCP) são finitos.

\section{Proposição 2.13 (Número máximo de soluções - caso geral) \\ $O$ (EiCP) tem no máximo $2^{n+1}-n-2$ soluções. $O(G E i C P)_{J}$ tem no máximo $(n-|J|+2) 2^{|J|}-|J|-2$ soluções.}

Prova : Considere inicialmente o $(\mathrm{GEiCP})_{J}$. Existem $2^{|J|}$ subconjuntos $I$ possíveis tais que $\bar{J} \subset I \subset\{1,2, \ldots, n\}$. Para cada subconjunto $I$ possível existem no máximo $|I|$ soluções distintas, que são os valores próprios de $B_{I I}^{-1} A_{I I}$. Portanto o número total de soluções é no máximo

$$
\begin{aligned}
\sum_{i=0}^{|J|}(|\bar{J}|+i)\left(\begin{array}{c}
|J| \\
i
\end{array}\right) & =(n-|J|) \sum_{i=0}^{|J|}\left(\begin{array}{c}
|J| \\
i
\end{array}\right)+\sum_{i=1}^{|J|} \sum_{k=i}^{|J|}\left(\begin{array}{c}
|J| \\
k
\end{array}\right) \\
& =(n-|J|) 2^{|J|}+\sum_{i=1}^{|J|}\left(2^{|J|-i+1}-1\right) \\
& =(n-|J|) 2^{|J|}+2^{|J|+1}-|J|-2 \\
& =(n-|J|+2) 2^{|J|}-|J|-2 .
\end{aligned}
$$

Como o (EiCP) é a instância particular do $(\mathrm{GEiCP})_{J}$ com $J=\{1,2, \ldots, n\}$, o número de soluções é no máximo

$$
(n-|J|+2) 2^{|J|}-|J|-2=2^{n+1}-n-2 .
$$


Os conjuntos completos de soluções do (EiCP) e do (GEiCP) podem ser obtidos por enumeração completa, como a seguir.

\section{Conjunto Completo de Soluçōes do (GEiCP) $J$}

1. Para cada subconjunto $I$ tal que $\bar{J} \subseteq I \subseteq\{1,2, \ldots, n\}$ compute o conjunto $\Lambda(I)$ de valores próprios de $B_{I I}^{-1} A_{I I}$.

2. Para cada $\bar{\lambda} \in \Lambda(I)$ tal que $\bar{\lambda}>0$, tente encontrar $x_{I}$ tal que

$$
\left\{\begin{array}{l}
A_{I I} x_{I}=\bar{\lambda} B_{I I} x_{I} \\
A_{J I} x_{I} \geq \bar{\lambda} B_{J I} x_{I} \\
x_{I \cap J} \geq 0 \\
\left\|x_{I}\right\|=1 .
\end{array}\right.
$$

Se isto for possível, então $x=\left(x_{I}, 0\right)$ é um valor próprio complementar geral associado ao valor próprio complementar geral $\bar{\lambda}$.

A corretude deste método é conseqüência direta da proposição 2.12. Este algoritmo também fornece uma condição necessária e suficiente para a viabilidade de um dado (GEiCP). A proposição seguinte mostra que qualquer condição necessária e suficiente para a viabilidade de um (GEiCP) será de alto custo computacional.

Proposição 2.14 (NP-completude da viabilidade do (GEiCP))

A viabilidade do $(\mathrm{GEiCP})_{J}$ é um problema de decisão NP-completo.

Prova : Qualquer solução $(\lambda, x)$ do $(\mathrm{GEiCP})_{J}$ satisfaz $x_{J} \geq 0$ e

$$
x^{t} A x=x^{t}(\lambda B x-w)=\lambda x^{t} B x-x^{t} w=\lambda x^{t} B x>0,
$$

pois $x \neq 0, \lambda>0$ e $B$ é positiva definida. Portanto, decidir se o (GEiCP) ${ }_{J}$ é viável é pelo menos tão difícil quando decidir se existe um $x$ tal que 
$x_{J} \geq 0$ e $x^{t} A x>0$, para uma dada matriz $A$. Este último problema é NP-completo (vide, por exemplo, [Murty 1988] teorema 2.20). Como a viabilidade do $(\mathrm{GEiCP})_{J}$ é claramente um problema NP, i.e. pode-se verificar em tempo polinomial se um dado par $(\lambda, x)$ é solução do $(\mathrm{GEiCP})_{J}$, segue o resultado da proposição.

Daí segue que resolver de fato o (GEiCP) é em geral um problema NP-difícil. Apesar disso, para algumas classes de matrizes a viabilidade do (EiCP) ou (GEiCP) correspondente pode ser verificada facilmente.

\section{Lema 2.15 (Caso de $A$ negativa semi-definida)}

Se A é negativa semi-definida, o $(G E i C P)_{J}$ correspondente é inviável.

Prova : Como $A$ é negativa semi-definida, segue que $A_{I I}$ é negativa semidefinida para todo $I \subseteq\{1,2, \ldots, n\}$. Portanto $x_{I}^{t} A_{I I} x_{I} \leq 0$, para qualquer $x$, e o problema é inviável.

\section{Lema 2.16 (Caso de $A$ positiva)}

Se $A$ é positiva $\left(A_{i j}>0, \forall i, j\right)$, e $B=I$, então o (EiCP) correspondente possui uma solução $\bar{\lambda}>0$, associada a um vetor próprio complementar $\bar{x}>0$. Mais ainda, se $A$ é simétrica, então a solução $\bar{\lambda}$ é única.

Prova : Como $A$ é positiva, qualquer solução viável $\bar{x} \neq 0$ tem que satisfazer $\bar{x}>0$, pois de outro modo $\bar{x}_{i}=0$ e $\bar{x} \neq 0$ implica $\vec{w}_{i}=\bar{\lambda} \bar{x}_{i}-A_{i} \bar{x}=$ $-A_{i} \bar{x}<0$. Por complementaridade e $\bar{x}>0$, tem-se $\bar{w}=0$, e portanto este (EiCP) é equivalente ao (EiP).

A existência de solução do (EiP) com uma matriz positiva é o Teorema de Perron (vide, por exemplo, [Ortega 1987] teorema 6.1.2). A unicidade no 
caso simétrico é verificada como segue. Seja $\hat{\lambda} \neq \bar{\lambda}$, e considere um vetor $\hat{x}$ tal que $A \hat{x}=\hat{\lambda} \hat{x}$. Então

$$
\bar{x}^{t} \hat{x}=\frac{1}{\bar{\lambda}}(A \bar{x})^{t} \hat{x}=\frac{1}{\bar{\lambda}} \bar{x}^{t} A^{t} \hat{x}=\frac{\hat{\lambda}}{\bar{\lambda}} \bar{x}^{t} \hat{x}
$$

e como $\hat{\lambda} \neq \bar{\lambda}$, tem-se $\bar{x}^{t} \hat{x}=0$. Como $\bar{x}>0, \hat{x}$ possui pelo menos uma componente negativa, e não pode ser solução do (EiP).

\section{Lema 2.17 (Caso de coluna com estrutura de sinal)}

Se existe um índice $j$ tal que $A_{j j}>0$ e $A_{i j} \leq 0, \forall i \neq j$, então $(\lambda, x)$ é uma solução do (EiCP), com $\lambda=A_{j j}$ e $x=e^{j}$ (j-ésimo vetor da base canônica).

\section{Prova : Trivial.}

Em particular esta propriedade vale para a classe importante de matrizes $\mathrm{K}$. Neste caso, $\lambda=A_{j j}$ é solução com $x=e^{j}$, para todos os índices $j \in\{1,2, \ldots, n\}$.

A seção seguinte dedica-se ao caso especial dos Problemas de Valores Próprios Complementares com matrizes simétricas. Algumas propriedades adicionais do (GEiCP) geral (não necessariamente simétrico) são apresentadas no apêndice.

\subsection{O Problema de Valores Próprios Complementar Simétrico}

Nesta seção as versões simétricas do (EiCP) e do (GEiCP) são estudadas. São derivadas algumas propriedades destes problemas, incluindo uma condição necessária e suficiente para viabilidade. Quando esta condição é verificada, o problema pode ser resolvido por uma variedade de métodos conhecidos em programação não-linear diferenciável.

Para clareza da exposição, os resultados para o (EiCP) e para o (GEiCP) são apresentados separadamente. 


\section{O (EiCP) Simétrico}

O (EiCP) simétrico é estreitamente relacionado ao problema clássico de valores próprios (GEiP). Como $x \neq 0$ para toda solução, a condição de complementaridade $w^{t} x=0$ pode ser reescrita como $x^{t}(\lambda B x-A x)=0$, ou equivalentemente,

$$
\lambda(x)=\frac{x^{t} A x}{x^{t} B x} .
$$

Este é o quociente de Rayleigh generalizado. Analogamente ao caso clássico, pontos de equilíbrio do quociente de Rayleigh no ortante não-negativo com $\lambda(x)>0$ são soluções do (EiCP).

Proposição 2.18 ((EiCP) simétrico como problema de otimização)

O (EiCP) simétrico é equivalente a

$$
(O E i C P) \begin{cases}\max & \lambda(x) \\ \text { s.a. } & x \in X=\left\{x \geq 0 \mid \mathbb{1}^{t} x=1\right\}\end{cases}
$$

no sentido que todo ponto de equilibrio $x$ do (OEiCP) $\operatorname{com} \lambda(x)>0$ é uma solução do $(E i C P)$.

Prova : O problema de otimização (OEiCP) é equivalente a

$$
\max _{x \in \mathbf{R}^{n}} \inf _{\substack{u \geq 0 \\ \alpha \in \mathbb{R}}} \frac{x^{t} A x}{x^{t} B x}+u^{t} x+\alpha\left(\mathbb{1}^{t} x-1\right) .
$$

Qualquer solução de equilíbrio deste problema satisfaz as condições de Kuhn-Tucker

$$
\left\{\begin{array}{l}
u+\alpha \mathbb{1}=\frac{2}{x^{t} B x}[\lambda(x) B-A] x \\
u \geq 0 \\
x \geq 0 \\
u^{t} x=0 \\
\mathbb{1}^{t} x=1 .
\end{array}\right.
$$


Fazendo o produto escalar da primeira equação por $x$ e notando que $u^{t} x=0, \mathbb{1}^{t} x=1$, e $\lambda(x)=\frac{x^{t} A x}{x^{t} B x}$, segue que $\alpha=0$. O sistema de equações resultante corresponde estreitamente ao (EiCP), com $u=\frac{2}{x^{t} B x} w$, e sem a restrição $\lambda>0$. Portanto qualquer ponto de equilíbrio do (OEiCP) com $\lambda(x)>0$ é uma solução do (EiCP).

\section{Observação 2.19 (Interpretação de $\alpha=0$ )}

$O$ resultado $\alpha=0$ na demonstração acima, apesar de aparentemente não intuitivo, pode ser motivado pela homogeneidade da função $\lambda(\cdot)$ e pela ortogonalidade entre $x$ e $\nabla \lambda(x)$ (lema 2.8). Considere, por exemplo, um ponto de equilibrio $x>0$ do (OEiCP). Pela homogeneidade de $\lambda(\cdot)$, sabe-se que a componente do gradiente $\nabla \lambda(x)$ em relação à semi-reta $\{\alpha x \mid \alpha \geq 0\}$ é nula; pelo equilíbrio em relação ao espaço afim $\left\{x \mid \mathbb{1}^{t} x=1\right\}$, tem-se que a componente projetada de $\nabla \lambda(x)$ neste espaço também é nula. Assim, $\nabla \lambda(x)=0$, e o multiplicador de Lagrange associado $a \mathbb{1}^{t} x=1$ deixa de ser necessário na combinação linear para expressar $\nabla \lambda(x)$.

\section{Observação 2.20 (Relação com NCPs simétricos)}

A proposição 2.18 é semelhante a um resultado clássico para problemas de complementaridade não-linear, baseado no seguinte Princípio de Simetria (vide [Ortega \& Rheinboldt 1970], teorema 4.1.6):

Teorema: Seja $F: \mathbb{R}^{n} \rightarrow \mathbb{R}^{n}$ continuamente diferenciável. Então $F$ é o gradiente de uma função $f: \mathbb{R}^{n} \rightarrow \mathbb{R}$ se, e somente se, o Jacobiano $\nabla F(x)$ é simétrico para todo $x \in \mathbb{R}^{n}$. Neste caso, $f$ pode ser escrita como

$$
f(x)=\int_{0}^{1} F(s x)^{t} x d s .
$$


Com posse deste resultado, conclui-se que o NCP

$$
\left\{\begin{array}{l}
x \geq 0 \\
F(x) \geq 0 \\
x^{t} F(x)=0
\end{array}\right.
$$

com $\nabla F(x)$ simétrico para todo $x$ é equivalente às condições de otimalidade de primeira ordem do problema de otimização

$$
\begin{cases}\min & f(x)=\int_{0}^{1} F(s x)^{t} x d s \\ \text { s.a. } & x \geq 0\end{cases}
$$

No caso em questão, parece difícil incorporar a restrição $\mathbb{1}^{t} x=1$ (ou $x \neq 0$ ) sem quebrar a simetria do problema. Pode-se constatar esta dificuldade ao considerar-se o NCP equivalente, obtido ao acrescentar uma variável auxiliar $\alpha$ satisfazendo

$$
\left\{\begin{array}{l}
w=(B-\mu A) x \\
\alpha=-1+\mathbb{1}^{t} x \\
w \geq 0 \\
x \geq 0 \\
w^{t} x=\alpha \mu=0
\end{array}\right.
$$

onde $\mu=\frac{1}{\lambda}$ em relação ao problema original. Neste contexto, as hipóteses do teorema clássico não se verificam.

A partir da reformulação do (EiCP) como um problema de Programação NãoLinear várias conclusões podem ser tiradas. A primeira delas é umạ, caracterização da viabilidade do (EiCP).

Proposição 2.21 (Viabilidade do (EiCP) simétrico)

$O(E i C P)$ simétrico é viável se, e somente se, existe $x \geq 0$ tal que $x^{t} A x>0$. 
Prova : Suponha, por um lado, que o (EiCP) é viável. Então, se $(\lambda, x)$ é uma solução viável qualquer, tem-se $x^{t} A x=\lambda x^{t} B x>0$, pois $x \neq 0$ e $B$ é positiva definida.

Suponha, por outro lado, que exista um $\hat{x} \geq 0$ tal que $\hat{x}^{t} A \hat{x}>0$. Como $\lambda(x)$ é uma função contínua (em $x \neq 0$ ) e o conjunto $X=\left\{x \geq 0 \mid \mathbb{1}^{t} x=1\right\}$ é compacto, existe um ponto $\bar{x} \in X$ tal que $\lambda(\bar{x}) \geq \lambda(x), \forall x \in X$. Este ponto satisfaz as condições de equilíbrio do (OEiCP) e, em particular, satisfaz $\lambda(\bar{x}) \geq \lambda(\hat{x})>0$. Portanto, $\bar{x}$ é uma solução do (EiCP).

Para a solução prática do (EiCP) simétrico, qualquer método de subida de gradiente, a partir de uma solução inicial $\hat{x} \in X$ tal que $\hat{x}^{t} A \hat{x}>0$, obtém um ponto de equilíbrio que é solução do problema. A não-convexidade da função objetivo não é realmente um problema, visto que o problema de otimização global (OEiCP) não precisa ser resolvido.

Algumas classes de matrizes $(A)$ que possuem uma tal solução inicial podem ser facilmente identificadas, por exemplo, matrizes copositivas não-nulas e, em particular, matrizes semi-definidas positivas não-nulas. Em geral, encontrar um tal ponto inicial é um problema NP-completo (proposição 2.14). Mas para uma classe muito grande de matrizes este problema é trivial, como mostra a proposição a seguir. Para. estas matrizes, um ponto inicial pode ser obtido por inspeção, e a solução do (EiCP) é obtida facilmente a partir daí. 
Proposição 2.22 (Obtenção de ponto inicial conveniente - casos fáceis)

Suponha que a matriz A satisfaz uma dentre as seguintes condições

1. $\exists i: A_{i i}>0$;

2. $\exists i, j: A_{i i}=0, A_{j j} \leq 0$ e $A_{i j}>0$;

3. $A \geq 0, A \neq 0$;

4. A é uma matriz $S$;

Então um ponto $\hat{x} \in X$ tal que $\hat{x}^{t} A \hat{x}>0$ pode ser facilmente obtido, e o (EiCP) correspondente é viável.

\section{Prova :}

1. Seja $\hat{x}=e^{i}$; então $\hat{x}^{t} A \hat{x}=A_{i i}=1$.

2. Seja $\hat{x}_{j}=1, \hat{x}_{i}=\frac{1-A_{j j}}{2 A_{i j}}$, e $\hat{x}_{k}=0, \forall k \neq i, j$; então

$$
\hat{x}^{t} A \hat{x}=A_{j j} x_{j}^{2}+2 A_{i j} x_{i} x_{j}=1
$$

3. Se $A \geq 0, A \neq 0$, então $x=\mathbb{1}$ é tal que $x^{t} A x>0$.

4. Se $A$ é uma matriz $\mathrm{S}$, então existe um $\hat{x} \geq 0$ tal que $A \hat{x}>0$. Logo $\hat{x}^{t} A \hat{x}>0$. Um vetor $\hat{x}$ nestas condições pode ser obtido resolvendo-se o seguinte problema linear:

$$
(P L) \begin{cases}\max & y \\ \text { s.a. } & A x-y \mathbb{1} \geq 0 \\ & \mathbb{1}^{t} x=1 \\ & x \geq 0 \\ & y \in \mathbb{R} .\end{cases}
$$


próprio positivo. Suponha, por contradição, que exista uma outra solução $(\mu, y)$ tal que $I_{y}=\left\{i \mid y_{i}>0\right\}=I_{x}$ e $\mu \neq \lambda$. Como $A$ é simétrica, $(\lambda-\mu) x_{I}^{t} y_{I}=y^{t} A x-x^{t} A y=0$. Mas isto contradiz $x_{I}>0$ e $y_{I}>0$. Portanto, cada subconjunto possível $I$ admite no máximo uma solução, o que significa que o número de soluções é limitado superiormente por $2^{n}-1$.

\section{O (GEiCP) Simétrico}

Os principais resultados da seção anterior podem ser refraseados no contexto do (GEiCP) simétrico. Uma vez mais a condição de complementaridade $x^{t} w=0$ permite a expressão de $\lambda$ como função de $x$,

$$
\lambda(x)=\frac{x^{t} A x}{x^{t} B x}
$$

Como $x$ não é necessariamente não-negativo (apenas $x_{J}$ é não-negativo), a condição $x \neq 0$ não pode ser substituída por $\mathbb{1}^{t} x=1$. A restrição não-convexa $\|x\|_{2}=1$ é adotada para fins teóricos, mas é posteriormente descartada do modelo computacional, que consegue manter esta restrição implicitamente. O símbolo $\|\cdot\|$, sem sub-índice, será usado para indicar a norma Euclideana.

Proposição 2.25 ((GEiCP) como problema de otimização)

$O(G E i C P)_{J}$ simétrico é equivalente a

$$
(O G E i C P)_{J} \begin{cases}\max & \lambda(x) \\ \text { s.a. } & x_{J} \geq 0 \\ & \|x\|=1\end{cases}
$$

no sentido que todo ponto de equilibrio $x$ em $(O G E i C P)_{J} \operatorname{com} \lambda(x)>0$ é uma solução do $(\mathrm{GEiCP})_{J}$. 
Prova : O problema de otimização $(\mathrm{OGEiCP})_{J}$ é equivalente a

$$
\max _{x \in \mathbb{R}^{n}} \inf _{\substack{u_{J} \geq 0 \\ u_{\bar{J}}=0 \\ \alpha \in \mathbb{R}}} \frac{x^{t} A x}{x^{t} B x}+u^{t} x+\alpha\left(x^{t} x-1\right) .
$$

Qualquer ponto de equilíbrio deste problema satisfaz as condições de Kuhn-Tucker

$$
\left\{\begin{array}{l}
u+\alpha x=\frac{2}{x^{t} B x}[\lambda(x) B-A] x \\
u_{J} \geq 0 \\
x_{J} \geq 0 \\
u_{J}^{t} x_{J}=0 \\
u_{\bar{J}}=0 \\
x^{t} x=1
\end{array}\right.
$$

onde novamente, tomando o produto interno da primeira equação por $x$, pode-se ver que o multiplicador de Lagrange $\alpha$ associado à restrição $x^{t} x=1$ tem valor 0 . Este sistema corresponde estreitamente ao $(\mathrm{GEiCP})_{J}, \operatorname{com} u=\frac{2}{x^{t} B x} w$, e sem a restrição $\lambda>0$, e assim segue o resultado.

A dificuldade do problema de otimização (OGEiCP) é revelada através da não-concavidade da função objetivo (problema de maximização) e da restrição nãoconvexa $\|x\|=1$. A não-concavidade da função objetivo não é um problema tão sério, visto que se busca apenas pontos de equilíbrio e não soluções globalmente ótimas, exatamente como no caso do (EiCP). O conjunto de pontos viáveis de (OGEiCP) é uma superfície esférica com restrições de sinal, e portanto métodos de projeção podem ser facilmente implementados através da renormalização dos iterados. Vide, por exemplo, o método projetado de máxima subida (steepest ascent) abaixo, e a proposição 2.28 . 
A condição de viabilidade do (GEiCP) é análoga àquela do (EiCP).

Proposição 2.26 (Viabilidade do (GEiCP) simétrico)

$O(G E i C P)_{J}$ simétrico é viável se, e somente se, existe um $x \in \mathbb{R}^{n}$ tal que $x_{J} \geq 0$ $e x^{t} A x>0$.

É claro que se o (EiCP) é viável, então o $(\mathrm{GEiCP})_{J}$ é viável. Mas a classe de matrizes para as quais o $(\mathrm{GEiCP})_{J}$ é viável pode ser maior do que aquela associada ao (EiCP). Por exemplo, o (GEiCP) ${ }_{J} \operatorname{com} n=2, J=\{1\}, B=I$ e

$$
A=\left[\begin{array}{cc}
0 & -\frac{1}{2} \\
-\frac{1}{2} & -1
\end{array}\right]
$$

é viável, pois $x=(2,-1)^{t}$ é tal que $x_{J}=2 \geq 0$ e $x^{t} A x=1>0$. Uma solução do problema é obtida $\operatorname{com} x=(0.8844,-0.3663)$ e $\lambda=0.2071$. No entanto, o (EiCP) correspondente é inviável, pois $-A$ é copositiva.

Para a solução prática do $(\mathrm{GEiCP})_{J}$, é necessário um ponto inicial satisfazendo $x_{J} \geq 0$ e $x^{t} A x>0$, bem como um método de subida de gradiente para o problema de otimização (OGEiCP).

A inicialização para a solução do $(\mathrm{GEiCP})_{J}$ é similar à do (EiCP). As heurísticas dadas pelos resultados 2.22, 2.15, 2.16 e 2.17 são aplicáveis também neste contexto, e a questão da viabilidade pode ser respondida definitivamente resolvendo o LCP geral abaixo. 


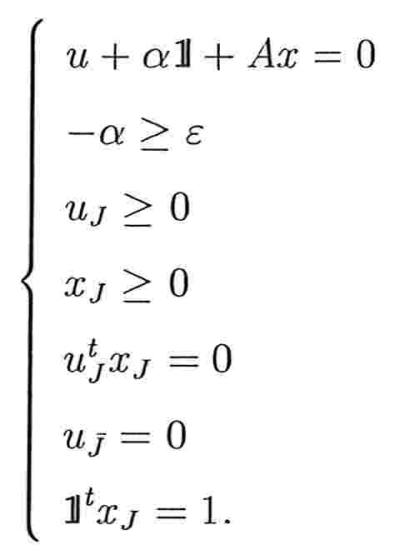

Neste problema a restrição $x \neq 0$ é substituída por $\mathbb{1}^{t} x_{J}=1$. Isto não foi feito no problema de otimização $(\mathrm{OGEiCP})_{J}$ para garantir a compacidade do conjunto de pontos viáveis, e simplificar a prova de convergência do método apresentado a seguir (proposição 2.28).

Dado um ponto inicial conveniente, um método de subida de gradiente pode ser facilmente adaptado para manter a restrição $\|x\|=1$ implicitamente, renormalizando cada iterado da seqüência gerada. Como um possível exemplo, o Método Projetado de Máxima Subida para a solução do (GEiCP) simétrico é apresentado a seguir, usando a regra de escolha de passo de Armijo.

\section{Método Projetado de Máxima Subida (MPMS) para o $(\mathrm{GEiCP})_{J}$ Simétrico}

0 . Seja $\varepsilon \in(0,1)$.

Seja $x^{0}$ tal que $x_{J}^{0} \geq 0,\left\|x^{0}\right\|=1$ e $\left(x^{0}\right)^{t} A x^{0}>0$.

Faça $k \longleftarrow 0$.

1. Compute $\nabla \lambda\left(x^{k}\right)=\frac{2}{\left(x^{k}\right)^{t} B x^{k}}\left[A-\lambda\left(x^{k}\right) B\right] x^{k}$, e $d^{k}$ por

$$
d_{j}^{k}= \begin{cases}0 & \text { se } j \in J, x_{j}^{k}=0 \text { e } \nabla \lambda\left(x^{k}\right)_{j}<0 \\ \nabla \lambda\left(x^{k}\right)_{j} & \text { caso contrário. }\end{cases}
$$


2. Sejam

$$
\begin{aligned}
& t_{k}=\min \left\{t \in \mathbb{N} \mid \lambda\left(x^{k}+\frac{1}{2^{t}} d^{k}\right)-\lambda\left(x^{k}\right) \geq \frac{\varepsilon}{2^{t}}\left\|d^{k}\right\|^{2}\right\}, \\
& \mu_{k}=\frac{1}{2^{t_{k}}}, \\
& y^{k+1}=x^{k}+\mu_{k} d^{k}, \\
& x^{k+1}=\frac{y^{k+1}}{\left\|y^{k+1}\right\|} .
\end{aligned}
$$

3. Faça $k \longleftarrow k+1$ e volte ao passo 1 .

\section{Observação 2.27 (Lipschitz-continuidade de $\nabla \lambda(\cdot)$ )}

A prova de convergência do método acima depende da propriedade de Lipschitzcontinuidade do gradiente $\nabla \lambda(\cdot)$ em $\{x \mid\|x\| \geq 1\}$, isto é, do fato que existe uma constante $L>0$ tal que

$$
\|\nabla \lambda(y)-\nabla \lambda(z)\| \leq L\|y-z\|
$$

para quaisquer $y, z \in\{x \mid\|x\| \geq 1\}$. A verificação formal desta propriedade é longa e foge ao escopo do trabalho. Na demonstração a seguir, supõe-se que $\nabla \lambda(\cdot)$ é Lipschitz-contínua com constante $L$.

\section{Proposição 2.28 (Convergência do MPMS)}

O Método Projetado de Máxima Subida converge para uma solução do (GEiCP) simétrico.

Prova : Primeiro observe que a direção $d^{k}$ computada no passo 2 ou é uma direção de subida, ou é zero. Isso ocorre porque

$$
\nabla \lambda\left(x^{k}\right)^{t} d^{k}=\left(d^{k}\right)^{t} d^{k}>0 \Longleftrightarrow d^{k} \neq 0
$$


A renormalização no passo 3 sempre pode ser feita, pois $y^{k+1} \neq 0$. Isso segue do fato que

$$
\left(x^{k}\right)^{t} d^{k}=\left(x^{k}\right)^{t} \nabla \lambda\left(x^{k}\right)=\frac{2}{\left(x^{k}\right)^{t} B x^{k}}\left[\left(x^{k}\right)^{t} A x^{k}-\lambda\left(x^{k}\right)\left(x^{k}\right)^{t} B x^{k}\right]=0 .
$$

Além disso, a renormalização preserva o valor da função objetivo,

$$
\lambda\left(x^{k+1}\right)=\lambda\left(\frac{y^{k+1}}{\left\|y^{k+1}\right\|}\right)=\lambda\left(y^{k+1}\right),
$$

bem como a restrição $x_{J}^{k+1} \geq 0$. Portanto, a seqüência $\left\{x^{k}\right\}$ está bem definida, e pertence ao compacto $\left\{x \mid x_{J} \geq 0,\|x\|=1\right\}$.

Para qualquer subseqüência convergente $x^{k} \longrightarrow x^{*}$, pela continuidade de $\nabla \lambda(\cdot)$ tem-se que $\nabla \lambda\left(x^{k}\right) \longrightarrow \nabla \lambda\left(x^{*}\right)$ e $d^{k} \longrightarrow d^{*}$, onde

$$
d_{j}^{*}= \begin{cases}0 & \text { se } j \in J, x_{j}^{*}=0 \text { e } \nabla \lambda\left(x^{*}\right)_{j}<0 \\ \nabla \lambda\left(x^{*}\right)_{j} & \text { caso contrário. }\end{cases}
$$

Prova-se a seguir que $d^{*}=0$.

Pela escolha de passo de Armijo, tem-se

$$
\lambda\left(y^{k+1}\right)-\lambda\left(x^{k}\right) \geq \frac{\varepsilon}{2^{t_{k}}}\left\|d^{k}\right\|^{2} .
$$

Além disso, pelo teorema do valor médio, existe um ponto $z \in \operatorname{conv}\left\{x^{k}, y^{k+1}\right\}$, tal que

$$
\begin{aligned}
\lambda\left(y^{k+1}\right)-\lambda\left(x^{k}\right) & =\mu_{k} \nabla \lambda(z)^{t} d^{k} \\
& =\mu_{k}\left[\nabla \lambda\left(x^{k}\right)+\nabla \lambda(z)-\nabla \lambda\left(x^{k}\right)\right]^{t} d^{k} \\
& =\mu_{k} \nabla \lambda\left(x^{k}\right)^{t} d^{k}-\mu_{k}\left[\nabla \lambda(z)-\nabla \lambda\left(x^{k}\right)\right]^{t} d^{k} .
\end{aligned}
$$

Notando que $\lambda\left(x^{k}\right)^{t} d^{k}=\left(d^{k}\right)^{t} d^{k}$, e usando a desigualdade de CauchySchwarz para o segundo termo, tem-se $\mu_{k} \nabla \lambda\left(x^{k}\right)^{t} d^{k}-\mu_{k}\left[\nabla \lambda(z)-\nabla \lambda\left(x^{k}\right)\right]^{t} d^{k} \geq \mu_{k}\left\|d^{k}\right\|^{2}-\mu_{k}\left\|\nabla \lambda(z)-\nabla \lambda\left(x^{k}\right)\right\|\left\|d^{k}\right\|$. 
Pela Lipschitz-continuidade de $\nabla \lambda(\cdot)$, e notando-se que $z \in \operatorname{conv}\left\{x^{k}, y^{k+1}\right\}$ e $y^{k+1}=x^{k}+\mu_{k} d^{k}$, tem-se

$$
\begin{aligned}
\lambda\left(y^{k+1}\right)-\lambda\left(x^{k}\right) & \geq \mu_{k}\left\|d^{k}\right\|^{2}-\mu_{k}\left\|\nabla \lambda(z)-\nabla \lambda\left(x^{k}\right)\right\|\left\|d^{k}\right\| \\
& \geq \mu_{k}\left\|d^{k}\right\|^{2}-\mu_{k} L\left\|z-x^{k}\right\|\left\|d^{k}\right\| \\
& \geq \mu_{k}\left\|d^{k}\right\|^{2}-\mu_{k} L\left\|y^{k+1}-x^{k}\right\|\left\|d^{k}\right\| \\
& =\mu_{k}\left(1-\mu_{k} L\right)\left\|d^{k}\right\|^{2} \\
& =\frac{1}{2^{t_{k}}}\left(1-\frac{L}{2^{t_{k}}}\right)\left\|d^{k}\right\|^{2}
\end{aligned}
$$

Note que, pela definição do passo de Armijo, ou $t_{k}=0$, e neste caso a escolha de Armijo implica

$$
\lambda\left(y^{k+1}\right)-\lambda\left(x^{k}\right) \geq \varepsilon\left\|d^{k}\right\|^{2}
$$

ou então $t_{k}>0 \mathrm{e}$

$$
\left(1-\frac{L}{2^{t_{k}-1}}\right)<\varepsilon
$$

pois, do contrário,

$$
\begin{aligned}
\left(1-\frac{L}{2^{t_{k}-1}}\right) \geq \varepsilon \Longrightarrow \lambda\left(x^{k}+\frac{1}{2^{t_{k}-1}}\right)-\lambda\left(x^{k}\right) & \geq \frac{1}{2^{t_{k}-1}}\left(1-\frac{L}{2^{t_{k}-1}}\right)\left\|d^{k}\right\|^{2} \\
& \geq \frac{\varepsilon}{2^{t_{k}-1}}\left\|d^{k}\right\|^{2}
\end{aligned}
$$

contradizendo a escolha de $t_{k}$. Assim, $\left(1-2 L \mu_{k}\right)<\varepsilon$, ou seja, $\varepsilon \mu_{k}>\varepsilon \frac{(1-\varepsilon)}{2 L}$. Substituindo-se esta desigualdade na definição do passo de Armijo, e considerando-se a possibilidade de $t_{k}=0$, tem-se

$$
\left\|d^{k}\right\|^{2} \leq \max \left\{\frac{1}{\varepsilon}, \frac{2 L}{\varepsilon(1-\varepsilon)}\right\}\left(\lambda\left(y^{k+1}\right)-\lambda\left(x^{k}\right)\right) .
$$

Prova-se a seguir que $\left\|y^{k+1}-x^{k}\right\| \longrightarrow 0$. Como $\left\|x^{k+1}-x^{k}\right\| \longrightarrow 0$, tem-se que $\theta^{k} \longrightarrow 0$, onde $\theta^{k}$ é o ângulo entre $x^{k+1}$ e $x^{k}$. 


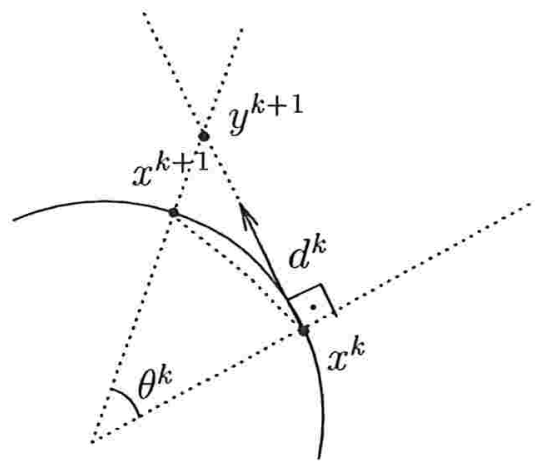

Portanto

$$
\cos \theta^{k}=\frac{\left\|y^{k+1}-x^{k}\right\|}{\left\|x^{k}\right\|}=\left\|y^{k+1}-x^{k}\right\| \longrightarrow 0 .
$$

Pela continuidade de $\lambda(\cdot)$, tem-se que $\lambda\left(y^{k+1}\right)-\lambda\left(x^{k}\right) \longrightarrow 0$. Pelo argumento anterior, $\left\|d^{k}\right\|^{2} \longrightarrow 0$, ou seja, $d^{*}=0$.

Como $\nabla \lambda\left(x^{*}\right)_{\bar{J}}=d_{\bar{J}}^{*}=0$ e $\nabla \lambda\left(x^{*}\right)_{J} \leq 0$, tem-se que $x=x^{*}, \alpha=0$ e $u=-\nabla \lambda\left(x^{*}\right)$ satisfazem as condições de Kuhn-Tucker do (OGEiCP) ${ }_{J}$, e $\operatorname{como} x^{*}$ satisfaz $\lambda\left(x^{*}\right) \geq \lambda\left(x^{0}\right)>0$, esta é uma solução do $(\mathrm{GEiCP})_{J}$.

O algoritmo acima foi escolhido para fins didáticos, pela clareza de exposição. Outras estratégias de escolha de passo na busca linear podem ser usadas, bem como outras direções de subida $d^{k}$ tais que $\nabla \lambda\left(x^{k}\right)^{t} d^{k}>0$.

\subsection{Experiência Computacional}

A fim de comparar a formulação original do (GEiCP) como NCP misto e a sua reformulação como problema de programação não-linear (OGEiCP), quanto à dificuldade relativa de resolução destes problemas, foram escolhidos dois pacotes comerciais dentro do sistema GAMS 2.5E: o pacote GAMS/PATH 4.3 para NCPs mistos, e o pacote GAMS/MINOS 5.5 para PNLs. 
Foram geradas 40 matrizes $A \in \mathbb{R}^{n \times n}$ aleatórias, 10 para cada valor de $n=10,20,30,40$ (a dimensão máxima para os testes é restrição da versão livre destes pacotes). Estas matrizes foram geradas de modo a garantir que $A_{11}>0$, o que fornece a solução inicial $x^{0}=(1,0, \ldots, 0)^{t} \geq 0$ tal que $\lambda\left(x^{0}\right)>0$. Os problemas correspondentes, tomando-se $B=I$ e $J=\{1,2, \ldots, n\}$, foram submetidos aos dois pacotes. Os testes foram executados em um Pentium II $333 \mathrm{MHz}$, rodando MS-Windows 98.

O algoritmo PATH [Dirkse \& Ferris 1995] é a implementação de um método de Newton amortecido para funções não-suaves, proposto em [Ralph 1994]. Este método é aplicado à reformulação do NCP misto como equação generalizada [Robinson 1979]. O algoritmo MINOS [Murtagh \& Saunders 1987] combina um método de gradiente reduzido [Wolfe 1962] e um método quasi-Newton [Murtagh \& Saunders 1978].

O resumo da experiência computacional encontra-se na tabela a seguir. As medidas de número de iterações e tempo de execução são valores médios sobre os 10 problemas na dimensão correspondente. Para o PATH, as iterações externas correspondem a passos de Newton, e as iterações internas são passos do método de Lemke para a construção de um caminho linear por trechos entre um iterado e o ponto de Newton correspondente [Dirkse \& Ferris 1995]. As colunas 4, 5 e 6 correspondem, respectivamente, ao número de problemas que o método PATH resolve sem necessidade de reinicialização, ao número de problemas resolvidos com o uso de até 3 reinicializações a partir de novos pontos iniciais gerados aleatoriamente, e ao número de problemas que o método não resolve. Note que a convergência do PATH depende de hipóteses sobre a inversibilidade local do Jacobiano da função $F(\cdot)$ que define o NCP, hipóteses estas que, em geral, não se verificam para o (GEiCP). 


\begin{tabular}{|c|c|c|c|c|c|c|c|c|}
\hline & \multicolumn{6}{|c|}{ РATH } & \multicolumn{2}{|c|}{ MINOS } \\
\hline 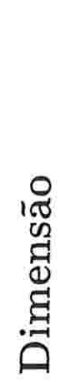 & 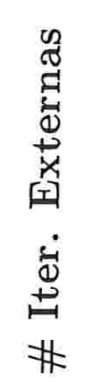 & 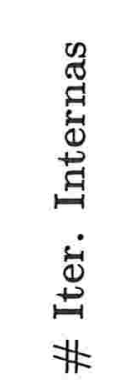 & $\begin{array}{l}\text { D } \\
0 \\
\text { D } \\
0 \\
0 \\
0 \\
\text { In } \\
\text { \# }\end{array}$ & 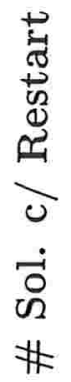 & 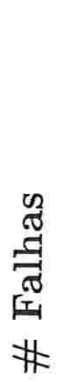 & 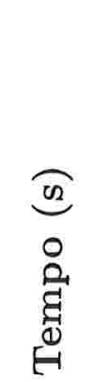 & 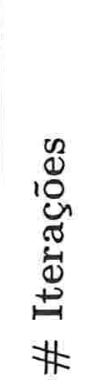 & 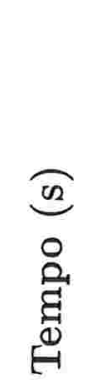 \\
\hline 10 & 33.6 & 165.5 & 7 & 2 & 1 & 0.30 & 16.8 & 0.04 \\
\hline 20 & 47.9 & 588.7 & 7 & 3 & 0 & 0.39 & 35.6 & 0.05 \\
\hline 30 & 77.1 & 958.8 & 4 & 6 & 0 & 0.71 & 52.5 & 0.09 \\
\hline 40 & 93.9 & 2418.7 & 3 & 6 & 1 & 1.30 & 67.8 & 0.15 \\
\hline
\end{tabular}

Note que o algoritmo PATH sem reinicialização, que corresponde ao algoritmo original de Ralph, falha entre $30 \%$ e $70 \%$ dos testes. Graças ao mecanismo de reinicialização e às heurísticas empregadas, uma parte considerável dos problemas finalmente é resolvida. Em um outro teste, 50 problemas aleatórios em dimensão $n=40$ foram gerados, como acima, e em 16\% destes o PATH não encontrou solução após 3 reinicializações. O algoritmo MINOS, em contrapartida, sempre encontrou solução, com tempos de execução entre 7.5 e 8.7 vezes mais rápidos.

Pode-se concluir, do estudo acima, que o algoritmo MINOS aplicado ao (OGEiCP) tem desempenho superior à do algoritmo PATH aplicado à formulação original do (GEiCP), tanto em relação ao tempo de execução, quanto em relação à robustez destes métodos para os problemas considerados.

\subsection{Conclusão e Trabalho Futuro}

Neste capítulo foram considerados os Problemas de Valores Próprios Complementares introduzidos em [Júdice, Costa, Figueiredo \& Martins 2000]. Mostrou-se que os 
casos simétricos são resolvidos com técnicas tradicionais em programação não-linear aplicadas ao quociente de Rayleigh, sujeito a restrições lineares. A inicialização do processo de otimização é em geral um problema NP-completo, mas para a maioria dos casos práticos o ponto inicial pode ser obtido por inspeção. A experiência computacional confirma que esta reformulação permite a resolução dos problemas simétricos em menor tempo e com maior robustez.

No apêndice a seguir, é apresentado um estudo preliminar no caso geral de matrizes assimétricas de ordem 2 , no que diz respeito à caracterização de viabilidade e número de soluções. A generalização destes resultados para matrizes assimétricas de qualquer ordem é tema de pesquisa em andamento.

Outro ponto de interesse consiste em encontrar técnicas de solução para a reformulação do (EiCP) apresentada no final do apêndice, como sistema de desigualdades polinomiais em $x$. 


\section{Apêndice: Caso Assimétrico}

Neste apêndice é considerado o (EiCP) $\operatorname{com} B=I$, sem a hipótese de simetria da ma$\operatorname{triz} A$. Mostra-se que a existência de solução quando $A$ é uma matriz $\mathrm{P}$, e o número de soluções estabelecido para o caso de matrizes simétricas também valem para matrizes assimétricas de ordem 2. Apresenta-se também uma possível reformulação do problema (GEiCP) como um sistema de desigualdades polinomiais na variável $x$ somente, e algumas experiências computacionais em MATLAB para esta reformulação.

\section{Lema 2.29 (Número máximo de soluçōes - dimensão 2)}

Se $A$ é uma matriz $2 \times 2$, então o (EiCP) tem no máximo 3 soluções.

Prova : O limitante superior dado pela proposição 2.13 é 4 . Se $A_{i i} \leq 0$ para algum $i$, ou se $A^{j} \geq 0$ para algum $j$, então as soluções associadas a $I=\{i\}$ ou $I=\{j\}$ são inviáveis, e portanto tem-se o resultado.

Se, por outro lado, $A_{i i}>0$ e $A_{i j} \leq 0$, para todo $i \neq j$, então as soluções associadas a $I=\{i\}$ e $I=\{j\}$ são viáveis. O caso restante é $I=\{1,2\}$, e este está associado a no máximo uma solução viável, como mostra-se a seguir.

O polinômio característico de $A$ $f(\lambda)=\operatorname{det}\left(\begin{array}{cc}\lambda-A_{11} & -A_{12} \\ -A_{21} & \lambda-A_{22}\end{array}\right)=\lambda^{2}-\left(A_{11}+A_{22}\right) \lambda+\left[A_{11} A_{22}-A_{12} A_{21}\right]$ tem no máximo duas raízes positivas. Além disso,

$$
f\left(A_{11}\right)=f\left(A_{22}\right)=-A_{12} A_{21} \leq 0
$$

e se $A_{12} A_{21}=0$, então ou $A_{11}$ ou $A_{22}$ é um valor próprio de $A$, fornecendo a mesma solução dos casos $I=\{i\}$ ou $I=\{j\}$. Portanto o (EiCP) tem no máximo 3 soluções. 
Suponha, por outro lado, que $A_{12}, A_{21}<0$. Então $f\left(A_{11}\right)=f\left(A_{22}\right)<0$, e existe um valor próprio $\lambda>\max \left\{A_{11}, A_{22}\right\}>0$. Um vetor próprio correspondente $x$ deve satisfazer

$$
\left\{\begin{array}{l}
\left(\lambda-A_{11}\right) x_{1}=A_{12} x_{2} \\
x_{1}+x_{2}=1 \\
x_{1}, x_{2} \geq 0
\end{array}\right.
$$

Isto é impossível, visto que $A_{12}<0$ e $\lambda>A_{11}$. Portanto este $\lambda$ não é solução do (EiCP), e o número máximo de soluções é 3 .

\section{Lema 2.30 (Viabilidade do (EiCP) com matriz P - dimensão 2)}

Se A é uma matriz $P$ de ordem 2, então o (EiCP) é viável.

Prova : Como $A \in P$, então $A_{i i}>0, \forall i$. Se existe algum $j \neq i$ tal que $A_{i j} \leq 0$, então segue o resultado. Do contrário, $A_{i j}>0, \forall i, j$, e $f\left(A_{11}\right)=f\left(A_{22}\right)=-A_{12} A_{21}<0$, onde, como antes, $f(\lambda)$ é o polinômio característico de $A$. Então existe algum valor próprio $\lambda>\max \left\{A_{11}, A_{22}\right\}>0$. Como $A_{12}>0$, o sistema

$$
\left\{\begin{array}{l}
\left(\lambda-A_{11}\right) x_{1}=A_{12} x_{2} \\
x_{1}+x_{2}=1 \\
x_{1}, x_{2} \geq 0
\end{array}\right.
$$

possui solução $\left(x_{1}, x_{2}\right)$. Esta solução satisfaz $A_{21} x_{1}=\left(\lambda-A_{22}\right) x_{2}$, pois $\lambda$ é um valor próprio de $A$. Portanto o (EiCP) é viável.

Considera-se a seguir uma formulação equivalente do $(\mathrm{GEiCP})_{J}$, obtida através da constatação de que as variáveis $\lambda$ e $w$ no sistema que define o (GEiCP) $J$ são determinadas pelo valor da variável $x$, e pela condição de complementaridade. 
Assim, $x^{t} w=0$ e $x \neq 0$ determinam o valor de $\lambda$ e $w$ como

$$
\begin{aligned}
\lambda(x) & =\frac{x^{t} A x}{x^{t} B x} \\
w(x) & =[\lambda(x) B-A] x \\
& =\frac{x^{t} A x}{x^{t} B x} B x-A x .
\end{aligned}
$$

O próximo resultado mostra que o $(\mathrm{GEiCP})_{J}$ é equivalente a encontrar um ponto $x$ satisfazendo um sistema de desigualdades polinomiais em $x$.

\section{Lema $2.31\left((\mathrm{GEiCP})_{J}\right.$ como sistema de polinômios em $\left.x\right)$}

$x$ é solução do $(G E i C P)_{J}$ se, e somente se, $x \neq 0, x_{J} \geq 0, x^{t} A x>0 e$

$$
P(x)=\left[\begin{array}{c}
\left(x^{t} A x\right) B_{J} x-\left(x^{t} B x\right) A_{J} x \\
\left(x^{t} A x\right) B_{\bar{J}} x-\left(x^{t} B x\right) A_{\bar{J}} x \\
\left(x^{t} B x\right) A_{\bar{J}} x-\left(x^{t} A x\right) B_{\bar{J}} x
\end{array}\right] \geq 0 .
$$

Prova : Note que a restrição $w_{J} \geq 0$ pode ser escrita como

$$
\left[\lambda(x) B_{J}-A_{J}\right] x=\frac{x^{t} A x}{x^{t} B x} B_{J} x-A_{J} x \geq 0,
$$

que é equivalente a

$$
\left(x^{t} A x\right) B_{J} x-\left(x^{t} B x\right) A_{J} x \geq 0 .
$$

A restrição $w_{\bar{J}}=0$ pode ser escrita de como análogo como

$$
\left\{\begin{array}{l}
\left(x^{t} A x\right) B_{\bar{J}} x-\left(x^{t} B x\right) A_{\bar{J}} x \geq 0 \mathrm{e} \\
\left(x^{t} A x\right) B_{\bar{J}} x-\left(x^{t} B x\right) A_{\bar{J}} x \leq 0,
\end{array}\right.
$$

o que leva à formulação acima.

A mesma reformulação aplicada ao (EiCP) corresponde a dizer que $x$ é solução se, e somente se, $x \neq 0, x \geq 0, x^{t} A x>0$ e

$$
\left(x^{t} A x\right) B x \geq\left(x^{t} B x\right) A x .
$$


Esta transformação reduz a dimensão do problema original, e poderia ser resolvida iterativamente através de linearizações sucessivas do mapa polinomial $P(x)$.

Vários testes aleatórios foram gerados em MATLAB, e todos foram resolvidos com sucesso pela rotina fmincon(), que utiliza um método do tipo Quasi-Newton para problemas de minimização com restrições gerais. O problema foi formulado em MATLAB como maximizar o quociente de Rayleigh sujeito às restrições acima, onde $x \neq 0$ foi substituído por $\mathbb{1}^{t} x_{J}=1$. A matriz $A$ foi gerada aleatoriamente com o cuidado de garantir que $a_{j j}>0$ para algum índice (aleatório) $j$. Isso fornece o ponto inicial $x^{0}=e^{j}$ tal que $\lambda\left(x^{0}\right)>0$. Os subconjuntos $J$ também foram gerados aleatoriamente. A tabela abaixo resume os testes executados.

\begin{tabular}{ccc} 
Dimensão & \# Testes & Tempo Médio (s) \\
\hline \hline 50 & 10 & 19.03 \\
100 & 10 & 58.18 \\
150 & 10 & 204.81 \\
\hline \hline
\end{tabular}

Finalmente, uma reformulação do (EiCP) como MPEC, que poderá ser útil na resolução do (EiCP) assimétrico, é obtida usando-se a variável auxiliar $y=\lambda x$, que permite formular o problema como

$$
\begin{cases}\min & \frac{1}{2}\|y-\lambda x\|^{2} \\ \text { s.a. } & w=B y-A x \\ & \mathbb{1}^{t} y-\lambda=0 \\ & x, y, w \geq 0, \lambda \geq \varepsilon \\ & x^{t} w=0\end{cases}
$$

onde $\varepsilon>0$ é um sub-estimador para a solução. Uma propriedade interessante desta formulação é o fato do conjunto viável ser um LCP geral, ao invés de um NCP. Por outro lado, uma solução deste problema só é solução do (EiCP) se o valor da função objetivo é 0 , ou equivalentemente, se vale a igualdade $y=\lambda x$. 


\section{Capítulo 3}

\section{Cortes Profundos Para o LCP}

O Problema de Complementaridade Linear é: dados uma matriz $M \in \mathbb{R}^{n \times n}$ e um vetor $q \in \mathbb{R}^{n}$, encontrar $z \in \mathbb{R}^{n}$ tal que

$$
(L C P)\left\{\begin{aligned}
z & \geq 0 \\
w(z) & \geq 0 \\
z^{t} w(z) & =0
\end{aligned}\right.
$$

onde $w(z)=M z+q$. Este problema tem muitas aplicações importantes em Engenharia, Economia e Ciência da Computação, visto que generaliza os Problemas de Programação Linear, Bilinear e Quadrática, e é uma ferramenta fundamental na solução de Problemas de Complementaridade Não-Linear, Problemas de Otimização Bilevel e Problemas de Programação Matemática com Restrições de Equilíbrio. Alguns livros-texto e artigos de revisão sobre o Problema de Complementaridade Linear são [Cottle, Pang \& Stone 1992, Murty 1988, Cottle 1976, Cottle \& Dantzig 1968, Eaves 1971, Júdice 1994, Júdice \& Mitra 1988].

Algoritmos eficientes via de regra requerem alguma propriedade especial da matriz que define o LCP. Classes de matrizes tais como matrizes copositivas, positivas (semi-)definidas e matrizes suficientes desempenham um papel fundamental 
na teoria de complementaridade, e os LCPs correspondentes possuem soluções algorítmicas rápidas. Por outro lado, algoritmos projetados para resolver qualquer tipo de LCP, independente de propriedades matriciais especiais, normalmente envolvem algum esquema de enumeração explícita ou implícita. Alguns trabalhos sobre abordagens enumerativas para o LCP são [Al-Khayyal 1987, Júdice \& Faustino 1988b, Sherali, Krishnamurty \& Al-Khayyal 1998], e suas respectivas referências.

Reformulações do LCP como problemas de otimização revelam métodos de solução alternativos para matrizes em geral. As reformulações convencionais requerem a minimização (global) de uma função não-convexa em um poliedro. Algumas reformulações e abordagens algorítmicas podem ser encontradas em [Mangasarian 1997, Pardalos \& Rosen 1988].

Dentre as abordagens algorítmicas para a solução de LCPs como problemas de otimização estão os métodos de planos de corte. Uma hipótese usual dos métodos de planos de corte é a compacidade do conjunto viável. LCPs com conjunto viável compacto são definidos por matrizes Não- $S_{0}$ (seção 3.2). A classe de matrizes $S_{0}$ contém a classe das matrizes semi-monótonas $\left(E_{0}\right)$, e conseqüentemente contém todas as classes "boas" de matrizes (incluindo matrizes copositivas, positivas semi-definidas e suficientes). As matrizes Não- $S_{0}$ pertencem ao grupo de matrizes para as quais não se conhece nenhum algoritmo eficiente (i.e., com bons resultados teóricos de convergência) que processe o LCP correspondente.

Uma estratégia mista de planos de corte e enumeração é proposta, por exemplo, em [Sherali, Krishnamurty \& Al-Khayyal 1996], onde o LCP é reformulado como um Problema de Programação Bilinear. Em [Ramarao \& Shetty 1984] o LCP é reformulado no contexto de Programação Disjuntiva, e um método de cortes profundos é desenvolvido, com cortes semelhantes aos usados em um método branch \& bound.

Em [Thoai \& Tuy 1983] é analisada a adaptação ao LCP de uma técnica de particionamento de cones para problemas de Programação Côncava 
[Thoai \& Tuy 1980]. A condição de complementaridade do LCP é substituída pela minimização de uma função côncava linear por trechos. São usados cortes de concavidade [Tuy 1964] nos subproblemas para obtenção de limitantes inferiores e superiores. A convergência do método é estabelecida para LCPs com conjunto viável limitado.

No presente trabalho é apresentada uma técnica de aprofundamento de cortes de concavidade para o Problema de Complementaridade Linear (LCP), baseada na reformulação do mesmo como uma família de Problemas de Programação Côncava. Esta técnica visa aumentar o volume da região cortada, reajustando os parâmetros que definem a função objetivo côncava a cada passo, e obtém cortes mais profundos do que o corte de Tuy sempre que possível, isto é, sempre que o corte de Tuy não é eficiente em relação à família de problemas considerada. São analisados os métodos de [Tuy 1964] e [Thoai \& Tuy 1983], baseados no uso de cortes de concavidade, e o impacto da técnica de aprofundamento nos mesmos. Detalhes de implementação são discutidos, tais como degenerescência, cortes mal-condicionados e abandono de cortes. Alguns testes computacionais são apresentados, a fim de revelar potencialidades e fraquezas de cada um dos métodos. Em geral, constata-se que estes métodos de solução do (LCP) não têm bom desempenho computacional, e não são adequados para problemas de médio e grande porte.

Este capítulo está estruturado como segue. Uma breve revisão de cortes de concavidade de Tuy é apresentada na seção 3.1, bem como os métodos de [Tuy 1964, Thoai \& Tuy 1983]. O aprofundamento dos cortes baseado na família de Problemas de Programação Côncava é o tema da seção 3.3. Tópicos de implementação e experiência computacional são discutidos na seção 3.4, seguidos por algumas observações finais. 


\subsection{Cortes de Concavidade e o Método de Tuy}

Seja $f: \mathbb{R}^{n} \longrightarrow \mathbb{R}$ uma função côncava. Sabe-se que uma condição equivalente à definição de concavidade é que $f(\cdot)$ satisfaça

$$
f\left(\sum_{i=1}^{k} \lambda_{i} x^{i}\right) \geq \sum_{i=1}^{k} \lambda_{i} f\left(x^{i}\right)
$$

para qualquer $k \in \mathbb{N}$ e qualquer $\lambda \in \mathbb{R}_{+}^{n}$ tal que $\mathbb{1}^{t} \lambda=1$, onde $\mathbb{1}=(1, \ldots, 1)^{t}$. Uma condição mais fraca do que a anterior está associada à propriedade de quaseconcavidade. $f(\cdot)$ é quase-côncava se satisfaz

$$
f\left(\sum_{i=1}^{k} \lambda_{i} x^{i}\right) \geq \min \left\{f\left(x^{i}\right) \mid i=1, \ldots, k\right\},
$$

para qualquer $k \in \mathbb{N}$ e qualquer $\lambda \in \mathbb{R}_{+}^{n}$ tal que $\mathbb{1}^{t} \lambda=1$. Qualquer função côncava é também quase-côncava, pois $\lambda \geq 0$ e $\mathbb{1}^{t} \lambda=1$ implicam

$$
\sum_{i=1}^{k} \lambda_{i} f\left(x^{i}\right) \geq \sum_{i=1}^{k} \lambda_{i} \min \left\{f\left(x^{i}\right) \mid i=1, \ldots, k\right\}=\min \left\{f\left(x^{i}\right) \mid i=1, \ldots, k\right\} .
$$

Considere o problema de programação côncava sobre um poliedro $X \subset \mathbb{R}^{n}$, definido abaixo.

\section{Definição 3.1 (Problema de Programação Côncava - PPC)}

$$
(P P C) \begin{cases}\min & f(x) \\ \text { s.a. } & x \in X .\end{cases}
$$

Suponha que $X$ é um poliedro limitado tal que $\operatorname{Int}(X) \neq \emptyset$, e seja $v^{0}$ um vértice não-degenerado com vizinhos $v^{1}, v^{2}, \ldots, v^{n}$. Se $a^{t} x=b$ é o hiperplano tal que 
$a^{t} v^{1}=a^{t} v^{2}=\ldots=a^{t} v^{n}=b$ e $a^{t} v^{0}<b$, então a desigualdade $a^{t} x \leq b$ define uma restrição (ou corte) do poliedro $X$ onde vale a relação

$$
f(x) \geq \min \left\{f\left(v^{i}\right) \mid i=0, \ldots, n\right\}
$$

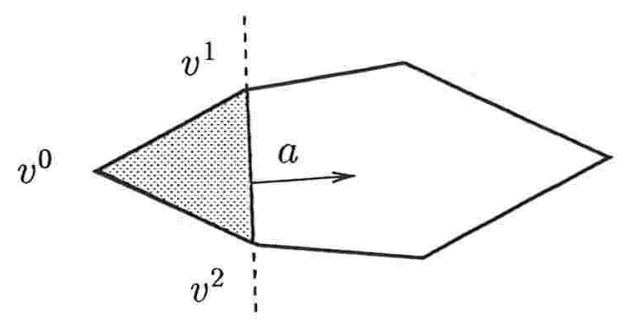

Para efeito de busca do mínimo global do problema (PPC), toda esta região pode ser eliminada do poliedro, desde que se conserve a informação do vértice $v^{i}$ que atinge o mínimo acima.

No caso especial em que $f\left(v^{0}\right)=\min \left\{f\left(v^{i}\right) \mid i=0, \ldots, n\right\}$, pode-se usar mais eficientemente a informação de concavidade da função $f(\cdot)$ para aumentar a região cortada. Basta escolher pontos $x^{i}=v^{0}+\theta_{i}\left(v^{i}-v^{0}\right)$ com $\theta_{i} \geq 1$ tais que $f\left(x^{i}\right)=f\left(v^{0}\right)$. Esta operação permite que outros vértices além de $v^{0}, v^{1}, v^{2}, \ldots, v^{n}$ sejam eliminados, sem a necessidade de inspeção dos mesmos.

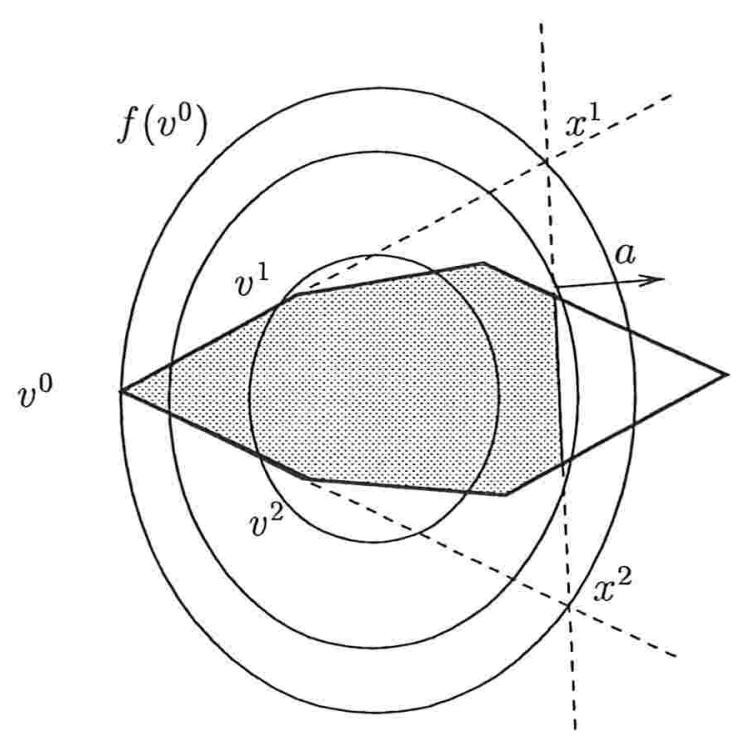




\section{Observação 3.2 (Aproximações cônicas e representação de cones)}

Note que as direções $x^{i}-v^{0}$ como acima definem uma aproximação cônica de $X$ em $v^{0}$

$$
X \subset v^{0}+\text { cone }\left\{x^{i}-v^{0} \mid i=1,2, \ldots, n\right\} .
$$

Dizer que $x-v^{0}$ está no cone $C=\operatorname{cone}\left\{x^{i}-v^{0} \mid i=1,2, \ldots, n\right\}$ é o mesmo que dizer que existe $\mu \geq 0$ tal que

$$
x-v^{0}=D \mu
$$

onde $D$ é a matriz com colunas $x^{1}-v^{0}, x^{2}-v^{0}, \ldots, x^{n}-v^{0}$. Supondo que $D$ seja não-singular, a equação anterior é equivalente a $\mu=D^{-1}\left(x-v^{0}\right)$, ou seja, todo $x-v^{0} \in C$ escreve-se de maneira única como

$$
x-v^{0}=\sum_{i=1}^{n} \mu_{i}\left(x^{i}-v^{0}\right) .
$$

com $\mu \in \mathbb{R}_{+}^{n}$. Neste caso, a condição $x-v^{0} \in C$ pode ser escrita como

$$
D^{-1} x \geq D^{-1} v^{0}
$$

O lema a seguir relaciona a hipótese $\operatorname{Int}(X) \neq \emptyset$ à representação da aproximação cônica discutida acima.

\section{Lema 3.3 (Caracterização $\operatorname{de} \operatorname{Int}(X) \neq \emptyset$ )}

Seja $v^{0}$ um vértice do poliedro $X \subset \mathbb{R}^{n}$ com vizinhos $v^{1}, v^{2}, \ldots, v^{n}$,

$$
C=\operatorname{cone}\left\{v^{i}-v^{0} \mid i=1,2, \ldots, n\right\}
$$

e $D$ a matriz com colunas $v^{1}-v^{0}, v^{2}-v^{0}, \ldots, v^{n}-v^{0}$. Säo equivalentes

1. D é não-singular;

2. $\operatorname{Int}(C) \neq \emptyset$;

3. $\operatorname{Int}(X) \neq \emptyset$. 
Prova : $[1 \Longleftrightarrow 2]$ Se $D$ é não-singular, tem-se $x-v^{0} \in C \Longleftrightarrow$ $D^{-1} x \geq D^{-1} v^{0}$. Se $x=v^{0}+\sum_{i=1}^{n}\left(v^{i}-v^{0}\right)$, então

$$
D^{-1} x=D^{-1} v^{0}+\sum_{i=1}^{n} e^{i}=D^{-1} v^{0}+e>D^{-1} v^{0} .
$$

Pela continuidade de $D^{-1}$, existe um $\varepsilon>0$ tal que $D^{-1}\left(y-v^{0}\right)>0$ para qualquer $y \in B(x, \varepsilon)$, e portanto $x-v^{0} \in \operatorname{Int}(C)$. Por outro lado, se $\operatorname{Int}(C) \neq \emptyset$, então $\operatorname{dim}(C)=n$, e como posto $(D)=\operatorname{dim}(C)=n$, segue que $D$ é não-singular.

$[2 \Longleftrightarrow 3]$ Se $x-v^{0} \in \operatorname{Int}(C)$, existe um $\varepsilon>0$ tal que $D^{-1}\left(y-v^{0}\right)>0, \forall y \in B(x, \varepsilon)$. Mais ainda, como $D^{-1}$ é linear, existe um $\bar{\alpha}>0$ tal que $D^{-1}\left(y-v^{0}\right)<\frac{\bar{\alpha}}{n}, \forall y \in B(x, \varepsilon)$. Então, para todo $y^{\prime} \in B\left(\frac{1}{\bar{\alpha}} x, \frac{\varepsilon}{\bar{\alpha}}\right)$,

$$
D^{-1}\left(y^{\prime}-v^{0}\right)=\frac{1}{\bar{\alpha}} D^{-1}\left(\bar{\alpha} y^{\prime}-v^{0}\right)
$$

e, como $\bar{\alpha} y^{\prime} \in B(x, \varepsilon)$, tem-se $D^{-1}\left(y^{\prime}-v^{0}\right)>0$ e $D^{-1}\left(y^{\prime}-v^{0}\right)<\frac{1}{n} 1$. Assim, se $\mu\left(y^{\prime}\right)=D^{-1}\left(y^{\prime}-v^{0}\right)$ então

$$
y^{\prime}=\left(1-\sum_{i=1}^{n} \mu_{i}(y)\right) v^{0}+\sum_{i=1}^{n} \mu_{i}(y)\left(v^{i}-v^{0}\right) \in X,
$$

pois $\sum_{i=1}^{n} \mu_{i}(y)<\sum_{i=1}^{n} \frac{1}{n}=1$. Deste modo, $B\left(\frac{1}{\bar{\alpha}} x, \frac{\varepsilon}{\bar{\alpha}}\right) \subset X$, e portanto $\operatorname{Int}(X) \neq \emptyset$. A implicação $[3 \Longrightarrow 2]$ é trivial porque $X-v^{0} \subset C$.

Voltando ao exemplo da página 85, note que, para cada $i$, a função $f^{j}(\theta)=f\left(v^{0}+\theta\left(v^{i}-v^{0}\right)\right)$ é côncava em $\theta$. Isto permite concluir que há somente duas possibilidades para o comportamento assintótico de $f^{j}(\theta)$.

Lema 3.4 (Comportamento assintótico de $f^{j}(\theta)=f\left(v^{0}+\theta\left(v^{i}-v^{0}\right)\right)$ )

Para cada $i=1, \ldots, n$, vale exatamente um dentre os casos

1a $\exists \bar{\theta} \geq 0: f\left(v^{0}+\bar{\theta}\left(v^{i}-v^{0}\right)\right)<f\left(v^{0}\right)$, 
2a $\nexists \bar{\theta} \geq 0: f\left(v^{0}+\bar{\theta}\left(v^{i}-v^{0}\right)\right)<f\left(v^{0}\right)$.

que correspondem, respectivamente, às condições

1b $\lim _{\theta \rightarrow \infty} f\left(v^{0}+\theta\left(v^{i}-v^{0}\right)\right)=-\infty$,

$2 \mathbf{b} \inf _{\theta \geq 0}\left\{f\left(v^{0}+\theta\left(v^{i}-v^{0}\right)\right)\right\}=f\left(v^{0}\right)$.

Prova : Prova-se a equivalência entre as condições de números correspondentes. No primeiro caso a implicação $[1 \mathrm{~b}] \Longrightarrow[1 \mathrm{a}]$ é trivial. Se vale $[1 \mathrm{a}]$, por outro lado, tem-se para todo $\theta>\bar{\theta}$,

$$
v^{0}+\bar{\theta}\left(v^{i}-v^{0}\right)=\frac{\bar{\theta}}{\theta}\left(v^{0}+\theta\left(v^{i}-v^{0}\right)\right)+\frac{\theta-\bar{\theta}}{\theta} v^{0},
$$

onde $\frac{\bar{\theta}}{\theta} \in[0,1]$. Então, pela concavidade de $f(\cdot)$,

$$
f\left(v^{0}+\bar{\theta}\left(v^{i}-v^{0}\right)\right) \geq \frac{\bar{\theta}}{\theta} f\left(v^{0}+\theta\left(v^{i}-v^{0}\right)\right)+\frac{\theta-\bar{\theta}}{\theta} f\left(v^{0}\right),
$$

de onde se conclui que, para todo $\theta>\bar{\theta}$,

$$
f\left(v^{0}+\theta\left(v^{i}-v^{0}\right)\right) \leq f\left(v^{0}\right)+\frac{\theta}{\bar{\theta}}\left(f\left(v^{0}+\bar{\theta}\left(v^{i}-v^{0}\right)\right)-f\left(v^{0}\right)\right) .
$$

Assim, como $f\left(v^{0}+\bar{\theta}\left(v^{i}-v^{0}\right)\right)-f\left(v^{0}\right)<0$,

$$
\lim _{\theta \rightarrow \infty} f\left(v^{0}+\theta\left(v^{i}-v^{0}\right)\right)=-\infty
$$

A verificação de $[2 \mathrm{a}] \Longleftrightarrow[2 \mathrm{~b}]$ é trivial: $f\left(v^{0}+\theta\left(v^{i}-v^{0}\right)\right) \geq f\left(v^{0}\right)$ para qualquer $\theta \geq 0$ é a definição de $\inf _{\theta \geq 0}\left\{f\left(v^{0}+\theta\left(v^{i}-v^{0}\right)\right)\right\}=f\left(v^{0}\right)$.

A discussão acima motiva as seguintes definições.

\section{Definição 3.5 (Mínimo local-*)}

Seja $X \subset \mathbb{R}^{n}$ um poliedro limitado e $v^{0}$ um vértice não-degenerado de $X$, com vizinhos $v^{1}, v^{2}, \ldots, v^{n}$. Seja $f: \mathbb{R}^{n} \longrightarrow \mathbb{R}$ uma função quase-côncava. Diz-se que $v^{0}$ é um mínimo local-* de $f(\cdot)$ em $X$ se

$$
f\left(v^{0}\right)=\min \left\{f\left(v^{i}\right) \mid i=0, \ldots, n\right\} .
$$




\section{Observação 3.6 (Mínimo local-* no contexto geral)}

A definição acima estende-se a poliedros ilimitados, considerando-se $v^{1}, \ldots, v^{i}$, $d^{k+1}, \ldots, d^{n}$, onde $v^{1}, \ldots, v^{k}$ são vértices vizinhos $e d^{k+1}, \ldots, d^{n}$ são raios extremais do cone de recessão. Neste caso a condição a ser verificada é

$$
f\left(v^{0}\right)=\inf \left\{f\left(v^{i}\right), f\left(v^{0}+\alpha d^{j}\right) \mid i=0, \ldots, k, j=k+1, \ldots, n, \alpha \geq 0\right\}
$$

A definição também estende-se a vértices degenerados, considerando-se o conjunto completo de vizinhos $v^{1}, \ldots, v^{k}, d^{k+1}, \ldots, d^{p}$ com $p>n$. Opta-se pela simplicidade ao se apresentar a definição especializada para o contexto deste trabalho.

\section{Definição 3.7 (Corte de concavidade)}

Seja $v^{0}$ um mínimo local- ${ }^{*}$ de uma função quase-côncava $f: \mathbb{R}^{n} \longrightarrow \mathbb{R}$ em um poliedro limitado $X \subset \mathbb{R}^{n}$, e seja $f^{*} \in \mathbb{R}$ tal que $f\left(v^{0}\right) \geq f^{*}$. Sejam $v^{1}, v^{2}, \ldots, v^{n}$ os vizinhos de $v^{0}$ em $X$, e defina

$$
\begin{aligned}
\theta_{i} & =\sup \left\{\theta \mid f\left(v^{0}+\theta\left(v^{i}-v^{0}\right)\right) \geq f^{*}\right\}, \\
I & =\left\{i \mid \theta_{i}<\infty\right\}, e \\
x^{i} & = \begin{cases}v^{0}+\theta_{i}\left(v^{i}-v^{0}\right), & \text { se } i \in I, \\
v^{i}, & \text { caso contrário. }\end{cases}
\end{aligned}
$$

Se $I \neq \emptyset$, o corte de concavidade (ou corte de Tuy) associado a $v^{0}$ e $f^{*}$ é a desigualdade $a^{t} x \geq b$ tal que $\|a\|=1, a^{t} v^{0}=b_{0}<b e$

$$
\begin{array}{ll}
a^{t} x^{i}=b, & \forall i \in I, \\
a^{t}\left(x^{i}-v^{0}\right)=0, & \forall i \notin I .
\end{array}
$$

Os pontos $x^{i}$ são chamados de extensões dos pontos $v^{i}$ (na direção $\left.v^{i}-v^{0}\right)$. 
Note que o corte está bem definido, porque como $\operatorname{Int}(X) \neq \emptyset$, o sistema de equações

$$
\begin{cases}a^{t}\left(x^{i}-v^{0}\right)=b-b_{0}, & \forall i \in I, \\ a^{t}\left(x^{i}-v^{0}\right)=0, & \forall i \notin I .\end{cases}
$$

admite solução (lema 3.3). Os valores $b$ e $b_{0}$ podem ser escolhidos livremente, desde que $b>b_{0}$, para garantir que $v^{0}$ está no semi-espaço eliminado pelo corte. A hipótese $\|a\|=1$ garante a unicidade da representação.

O que esta definição apresenta de novo em relação ao exemplo motivador é a possibilidade de cortes paralelos às arestas nas quais $f(\cdot)$ não decresce, como a seguir.

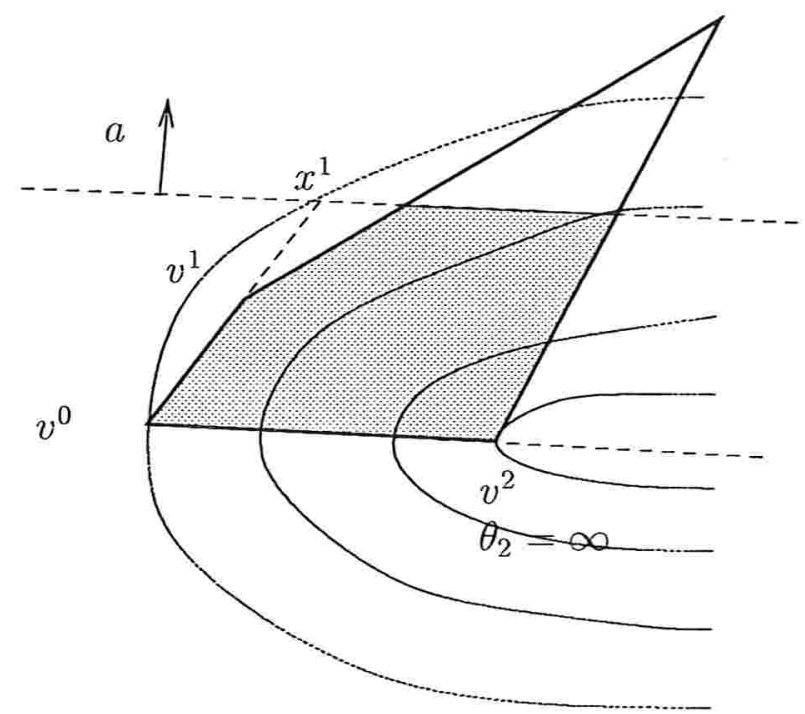

Esta figura também ilustra a seguinte caracterização dos pontos cortados.

\section{Lema 3.8 (Caracterização do corte)}

Se $x \in X$, então

$$
a^{t} x \leq b \Longleftrightarrow x \in \operatorname{conv}\left\{v^{0}, x^{i} \mid i \in I\right\}+\operatorname{cone}\left\{x^{i}-v^{0} \mid i \notin I\right\}
$$

Prova: Seja $\mu \geq 0$ tal que $x=v^{0}+\sum_{i=1}^{n} \mu_{i}\left(x^{i}-v^{0}\right)$. Fazendo o produto escalar por $a$ tem-se $a^{t} x=b_{0}+\sum_{i=1}^{n} \mu_{i} a^{t}\left(x^{i}-v^{0}\right)=b_{0}+\sum_{i \in I} \mu_{i}\left(b-b_{0}\right)$. 
Como $a^{t} x \leq b$, tem-se $\sum_{i \in I} \mu_{i}\left(b-b_{0}\right) \leq b-b_{0}$, e portanto

$$
\sum_{i \in I} \mu_{i} \leq 1
$$

Assim,

$$
x=\left[\left(1-\sum_{i \in I} \mu_{i}\right) v^{0}+\sum_{i \in I} \mu_{i} x^{i}\right]+\sum_{i \notin I} \mu_{i}\left(x^{i}-v^{0}\right),
$$

e isso prova o resultado.

Antes de provar o resultado fundamental que todo ponto $x$ cortado satisfaz $f(x) \geq f^{*}$, é necessário estabelecer um lema a respeito das direções das arestas paralelas ao corte de concavidade.

\section{Lema 3.9 (Comportamento de $f(\cdot)$ em direções paralelas ao corte)}

Seja $a^{t} x \geq b$ o corte de concavidade associado a $v^{0}$ e $f^{*}$ como na definição 3.7, e seja $\bar{x} \in \mathbb{R}^{n}$ tal que $f(\bar{x}) \geq f^{*}$. Então, para todo $\mu \geq 0$,

$$
f\left(\bar{x}+\sum_{i \notin I} \mu_{i}\left(x^{i}-v^{0}\right)\right) \geq f^{*} .
$$

Prova : Para cada $m \in \mathbb{N}_{+}, f\left(v^{0}+m \sum_{i \notin I} \mu_{i}\left(x^{i}-v^{0}\right)\right) \geq f^{*}$, pela definição do conjunto $I$. Defina

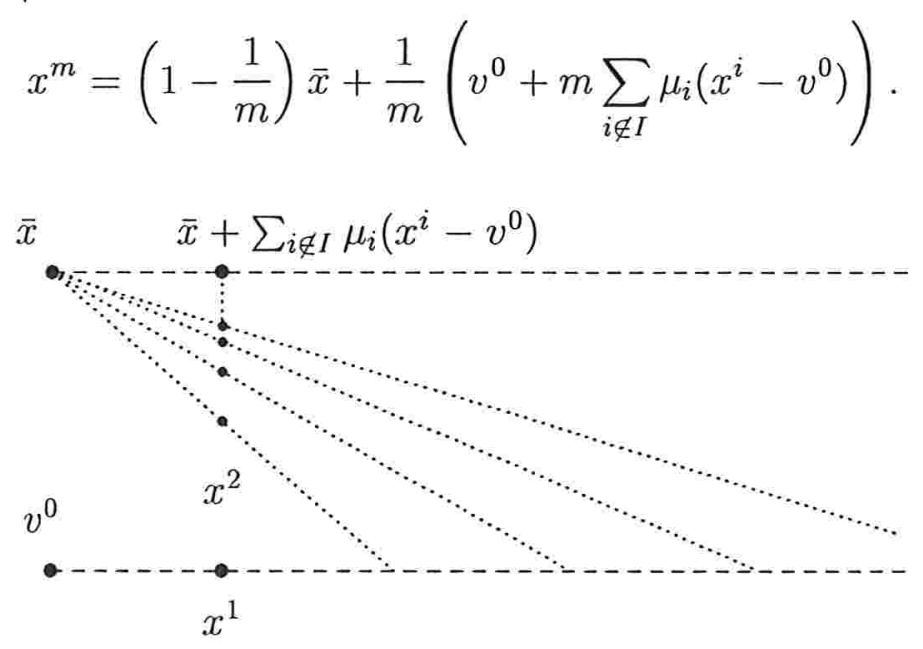


Como $f(\cdot)$ é côncava, e em particular quase-côncava, vale que $f\left(x^{m}\right) \geq f^{*}$.

Além disso, como $x^{m}=\bar{x}+\sum_{i \notin I} \mu_{i}\left(x^{i}-v^{0}\right)+\frac{1}{m}\left(v^{0}-\bar{x}\right)$, segue que

$$
\lim _{m \rightarrow \infty} x^{m}=\bar{x}+\sum_{i \notin I} \mu_{i}\left(x^{i}-v^{0}\right),
$$

e pela continuidade da $f(\cdot)$,

$$
f\left(\bar{x}+\sum_{i \notin I} \mu_{i}\left(x^{i}-v^{0}\right)\right) \geq f^{*}
$$

Proposição 3.10 (Corretude do corte de concavidade)

Seja $a^{t} x \geq b$ o corte de concavidade associado a $v^{0}$ e $f^{*}$, como na definição 3.7. Para todo ponto $x \in X$ tal que $a^{t} x \leq b$, tem-se $f(x) \geq f^{*}$.

Prova : Pelo lema 3.8, seja $\mu \geq 0$ tal que $\sum_{i \in I} \mu_{i} \leq 1$ e

$$
x=\left[\left(1-\sum_{i \in I} \mu_{i}\right) v^{0}+\sum_{i \in I} \mu_{i} x^{i}\right]+\sum_{i \notin I} \mu_{i}\left(x^{i}-v^{0}\right) .
$$

Pela quase-concavidade de $f(\cdot)$, segue que

$$
f\left(\left(1-\sum_{i \in I} \mu_{i}\right) v^{0}+\sum_{i \in I} x^{i}\right) \geq \min \left\{f\left(v^{0}\right), f\left(x^{i}\right) \mid i=1,2, \ldots, n\right\} \geq f\left(v^{0}\right) \geq f^{*}
$$

e, pelo lema $3.9, f(x) \geq f^{*}$.

Note que se $I=\left\{i \mid \theta_{i}<\infty\right\}=\emptyset$, então o corte de concavidade não está definido em 3.7. Isso explica-se pela proposição a seguir.

\section{Proposição 3.11 (Esvaziamento do poliedro I)}

Seja $v^{0}$ um mínimo local-* de $f(\cdot)$ em $X$ como na definição 3.\%. Se $I=\left\{i \mid \theta_{i}<\infty\right\}=\emptyset$, então $v^{0}$ é um minimo global de $f(\cdot)$ em $X$. 
Prova : Se $I=\emptyset$, pelo lema 3.9 tem-se $f\left(v^{0}+\sum_{i=1}^{n} \mu_{i}\left(x^{i}-v^{0}\right)\right) \geq f\left(v^{0}\right)$ para qualquer $\mu \geq 0$, e como $X \subset v^{0}+\operatorname{cone}\left\{x^{i}-v^{0} \mid i=1,2, \ldots, n\right\}$,

$$
f\left(v^{0}\right)=\min \{f(x) \mid x \in X\}
$$

De fato, quando $I=\emptyset$, não é possível construir um corte paralelo às $n$ arestas $x^{i}-v^{0}$, pois neste caso a única solução de

$$
a^{t}\left(x^{i}-v^{0}\right)=0, i=1,2, \ldots, n
$$

é $a=0$, e conseqüentemente $b=0$. Assim, não é possível satisfazer a condição $a^{t} v^{0}=b_{0}<0$.

Outro caso onde o poliedro é esvaziado pelo corte de Tuy é dado a seguir.

Proposição 3.12 (Esvaziamento do poliedro II)

Seja $v^{0}$ um mínimo local- ${ }^{*}$ de $f(\cdot)$ em $X$ como na definição 3.7, e suponha que o corte de Tuy $a^{t} x \geq b$ está definido. Se não existe nenhum vértice $v \in X$ tal que $a^{t} v>b$, então $v^{0}$ é um mínimo global de $f(\cdot)$ em $X$.

Prova : Basta notar que se não existe nenhum vértice de $X$ tal que $a^{t} v>$ $b$, então, como $X$ é limitado, a solução ótima $x^{*}$ de $\max _{x \in X} a^{t} x$ satisfaz $a^{t} x^{*} \leq b$. Logo, $a^{t} x \leq b$ para todo $x \in X$ e, pela proposição 3.10 , $f\left(v^{0}\right)=\min _{x \in X} f(x)$.

Implícita na definição de cortes de concavidade está uma idéia algorítmica muito simples, proposta em [Tuy 1964]. 
Método de Tuy (somente cortes de concavidade)

0 . Faça $k \leftarrow 0$ e seja $X^{0}=X$. Faça $f^{*} \leftarrow \infty$.

1. Encontre um mínimo local-* $v^{0}$ de $f(\cdot)$ em $X^{k}$. Sejam $v^{1}, v^{2}, \ldots, v^{n}$ seus vizinhos. Se $f\left(v^{0}\right)<f^{*}$ faça $f^{*} \leftarrow f\left(v^{0}\right)$ e $v^{*}=v^{0}$.

2. Compute, para cada $i=1,2, \ldots, n$,

$$
\theta_{i}=\sup \left\{\theta \mid f\left(v^{0}+\theta\left(v^{i}-v^{0}\right)\right) \geq f^{*}\right\}
$$

Se $I=\left\{i \mid \theta_{i}<\infty\right\}=\emptyset$, pare. $v^{*}$ é solução ótima de (PPC), com valor ótimo $f^{*}$.

3. Calcule o corte de concavidade $a^{t} x \geq b$ da definição 3.7 , e seja $x^{*}$ a solução ótima de $\max _{x \in X^{k}} a^{t} x$. Se $a^{t} x^{*} \leq b$, pare. $v^{*}$ é solução ótima de (PPC), com valor ótimo $f^{*}$.

4. Seja $X^{k+1}=X^{k} \cap\left\{x \mid a^{t} x \geq b\right\}$. Faça $k \leftarrow k+1$ e volte ao passo 1 .

Este algoritmo gera uma seqüência de poliedros encaixados $X^{0} \supset X^{1} \supset \cdots \supset$ $X^{k} \supset \cdots$ com a propriedade de que o mínimo local-* calculado em $X^{k}$ é inviável em $X^{k+1}$. Pela proposição 3.10 , sabe-se que ao final do passo 4 do algoritmo

$$
\min \left\{f(x) \mid x \in X^{k}\right\}=\min \left\{f\left(v^{*}\right), f(x) \mid x \in X^{k+1}\right\} .
$$

Por construção, os valores $f^{*}=f\left(v^{*}\right)$ no passo 2 formam uma seqüência não-crescente, e assim para qualquer $k \in \mathbb{N}$,

$$
\min \{f(x) \mid x \in X\}=\min \left\{f\left(v^{*}\right), f(x) \mid x \in X^{k}\right\}
$$

\section{Proposição 3.13 (Critério de parada do Método de Tuy)}

Se o Método de Tuy termina em um número finito de passos, então $v^{*}$ é solução ótima de (PPC), com valor ótimo $f^{*}$. 
Prova : O algoritmo possui dois critérios de parada, nos passos 2 e 3 . Suponha inicialmente que o algoritmo parou no passo 2, satisfazendo $I=\left\{i \mid \theta_{i}<\infty\right\}=\emptyset$. Então, pela proposição 3.11, $v^{0}$ é mínimo global do problema $\min _{x \in X^{k}} f(x)$, e portanto

$$
\min \{f(x) \mid x \in X\}=\min \left\{f^{*}, f(x) \mid x \in X^{k}\right\}=\min \left\{f^{*}, f\left(v^{0}\right)\right\}=f^{*} .
$$

Se, por outro lado, o algoritmo terminou no passo 3, satisfazendo $a^{t} x \leq b, \forall x \in X^{k}$, então $f^{*} \leq f(x), \forall x \in X^{k}$, pela proposição 3.10. Portanto,

$$
\min \{f(x) \mid x \in X\}=\min \left\{f^{*}, f(x) \mid x \in X^{k}\right\}=f^{*}
$$

Em [Horst \& Tuy 1993] provou-se que se existe um $\varepsilon>0$ tal que os cortes gerados pelo algoritmo satisfazem

$$
b-b_{0}>\varepsilon
$$

em todos os passos, então o algoritmo termina em um número finito de passos com a solução ótima do (PPC). No entanto, não é possível garantir, por construção, que a seqüência de cortes possui a propriedade acima, e o algoritmo não possuo nenhuma propriedade de convergência em geral.

Por curiosidade, pode-se constatar que em dimensão 2 o algoritmo sempre resolve o (PPC), pois é fácil verificar que cada corte elimina pelo menos 3 vértices e gera no máximo 2 novos vértices. Assim, se $V(X)$ indica o conjunto de vértices de $X$

$$
\left|V\left(X^{k+1}\right)\right|<\left|V\left(X^{k}\right)\right|
$$

e, portanto, o algoritmo termina em no máximo $|V(X)|-n$ iterações. 
Note que a mecânica do algoritmo depende da hipótese tácita de que sempre é possível encontrar um mínimo local-* do poliedro $X^{k}$ no passo 1 . O procedimento usual para este fim é

\section{Busca de Mínimo Local-* em $X^{k}$}

0 . Seja $v$ um vértice qualquer em $X^{k}$.

1. Enquanto $f(v)>f(u)$ para algum vizinho $u$ de $v$, faça $v \leftarrow u$.

No presente contexto, isso exige encontrar um mínimo local-* nãodegenerado, e a introdução sucessiva de cortes de concavidade pode tornar esta tarefa mais difícil, senão impossível. Note que o algoritmo acima ainda pode funcionar, mesmo passando por vértices degenerados no caminho até o mínimo local-*. O problema surge quando um vértice degenerado é um mínimo local-* no sentido da observação 3.6. Nesta situação, é necessário adaptar a definição de cortes de concavidade para a situação em que $v^{0}$ tem $p>n$ vizinhos.

Uma adaptação possível é considerar o problema linear

$$
\left\{\begin{array}{lll}
\min & \sum_{i \in I} r_{i}+\sum_{i \notin I} a^{t}\left(x^{i}-v^{0}\right) & \\
\text { s.a. } & a^{t} v^{0}=b-1 & \\
& a^{t} x^{i}-r_{i}=b & i \in I \\
& a^{t}\left(x^{i}-v^{0}\right) \geq 0 & \\
& r \geq 0 . &
\end{array}\right.
$$

O corte $a^{t} x \geq b$ definido pela solução deste problema corta o vértice $v^{0}$ (pois $\left.a^{t} v^{0}=b-1\right)$ e além disso passa pelos pontos

$$
\begin{array}{ll}
v^{0}+\frac{1}{1+r_{1}}\left(x^{i}-v^{0}\right), & i \in I \\
v^{0}+\frac{1}{a^{t}\left(x^{i}-v^{0}\right)}\left(x^{i}-v^{0}\right), & i \notin I \text { tal que } a^{t}\left(x^{i}-v^{0}\right)>0,
\end{array}
$$


pois, no primeiro caso,

$$
a^{t}\left(v^{0}+\frac{1}{1+r_{1}}\left(x^{i}-v^{0}\right)\right)=(b-1)+\frac{1}{1+r_{1}}\left(b+r_{i}-(b-1)\right)=b-1+\frac{1+r_{1}}{1+r_{1}}=b
$$

e, no segundo caso,

$$
a^{t}\left(v^{0}+\frac{1}{a^{t}\left(x^{i}-v^{0}\right)}\left(x^{i}-v^{0}\right)\right)=b-1+\frac{1}{a^{t}\left(x^{i}-v^{0}\right)} a^{t}\left(x^{i}-v^{0}\right)=b .
$$

Além disso o corte é trivialmente paralelo às direções $x^{i}-v^{0}$ tais que $a^{t}\left(x^{i}-v^{0}\right)=0$. A função objetivo do problema tenta trazer o corte para perto dos pontos $x^{i}(i \in I)$ e ao mesmo tempo tornar o corte "mais próximo de ser paralelo" às direções $\left(x^{i}-v^{0}\right)$ para $i \notin I$.

Um problema mais sério em relação à degenerescência é construir o conjunto de vizinhos $v^{1}, v^{2}, \ldots, v^{p}$ a partir de um vértice degenerado $v^{0}$. Note que sem isso não é possível afirmar que $v^{0}$ é um mínimo local-* no algoritmo de busca anterior, e nem sequer é possível formular o PL acima.

A primeira idéia que surge é enumerar as bases que representam $v^{0}$ e tomar a união dos vizinhos $v^{i}$ distintos de $v^{0}$ obtidos a partir de cada representação básica. A dificuldade neste caso pode ser ilustrada pelo exemplo a seguir. Considere o poliedro em $\mathbb{R}^{n}$ definido pelo sistema

$$
\begin{gathered}
{\left[\begin{array}{cccc}
-1 & 10 & \cdots & 10 \\
10 & -1 & & 10 \\
\vdots & & \ddots & \vdots \\
10 & 10 & \cdots & -1 \\
-1 & -1 & \cdots & -1
\end{array}\right] x \geq\left[\begin{array}{c}
0 \\
0 \\
\vdots \\
0 \\
-1
\end{array}\right]} \\
x \geq 0
\end{gathered}
$$

e considere o vértice $v^{0}=0$ representado pela base associada às variáveis residuais. 


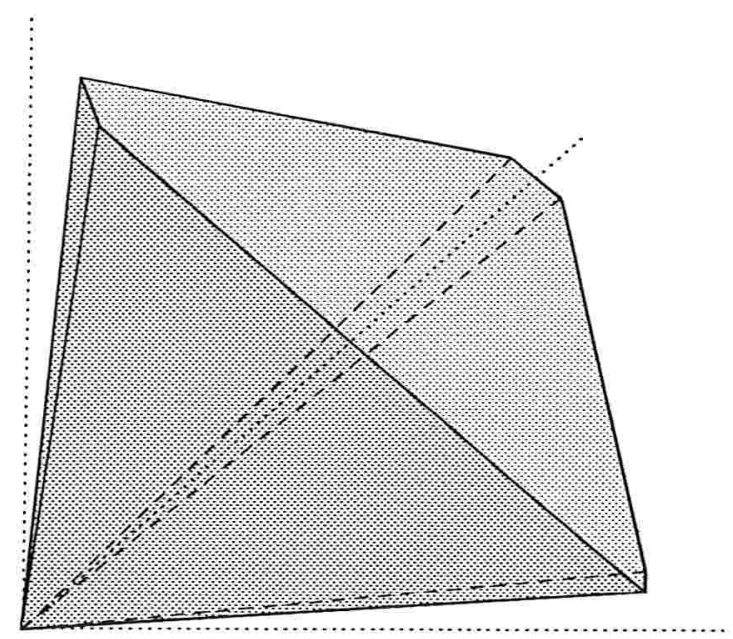

É fácil ver que as $n$ direções correspondentes à entrada de $x^{1}, x^{2}, \ldots, x^{n}$ na base são todas degeneradas, no sentido de permitir apenas o passo de tamanho 0. Esta base portanto não permite calcular nenhum vizinho $v^{i} \neq v^{0}$. Note ainda que neste exemplo a origem possui $2 n$ vizinhos, mas pode ser representada por $2^{n}$ bases. Em geral não é possível afirmar que $v^{1}, v^{2}, \ldots, v^{p}$ é o conjunto total de vizinhos de $v^{0}$ sem enumerar todas as bases que representam $v^{0}$.

A não garantia de convergência e as dificuldades com vértices degenerados, somadas à dificuldade numérica inerente ao aumento na descrição dos poliedros $X^{k}$, leva à consideração deste método como uma heurística, através da fixação de um limite máximo de iterações. Assim, se o algoritmo termina antes de atingir o limite máximo, a solução é um mínimo global para o problema (PPC); do contrário, a solução fornece um limitante superior para o valor ótimo.

Também em [Tuy 1964] é proposto um método que tenta explorar mais a fundo a concavidade da $f(\cdot)$. A essência do método é construir o corte de concavidade $a^{t} x \geq b$, e resolver o PL $\max _{x \in X} a^{t} x$ a fim de buscar o ponto $v^{n+1}$ em $X$ mais distante do corte. Se $a^{t} v^{n+1} \leq b$, o problema acabou. Se $f\left(v^{n+1}\right)<f^{*}$ então atualiza-se $f^{*}$, e uma extensão $x^{n+1}=v^{0}+\theta_{n+1}\left(v^{n+1}-v^{0}\right)$ de $v^{n+1}$ é calculada com $\theta_{n+1}=\sup \left\{\theta \mid f\left(v^{0}+\theta\left(v^{n+1}-v^{0}\right)\right) \geq f^{*}\right\}$. De posse deste novo ponto cria-se, para 
cada $i=1,2, \ldots, n$, um subproblema de PL usando o corte de concavidade definido pelos pontos $\left\{x^{n+1}, x^{j} \mid j \neq i\right\}$ cortando $x^{i}$. No exemplo abaixo, o primeiro corte calculado passa pelos pontos $\left(x^{1}, x^{2}\right)$. A solução do PL correspondente é $v^{3}$, e os subproblemas gerados estão associados a $\left(x^{1}, x^{3}\right)$ e $\left(x^{2}, x^{3}\right)$.

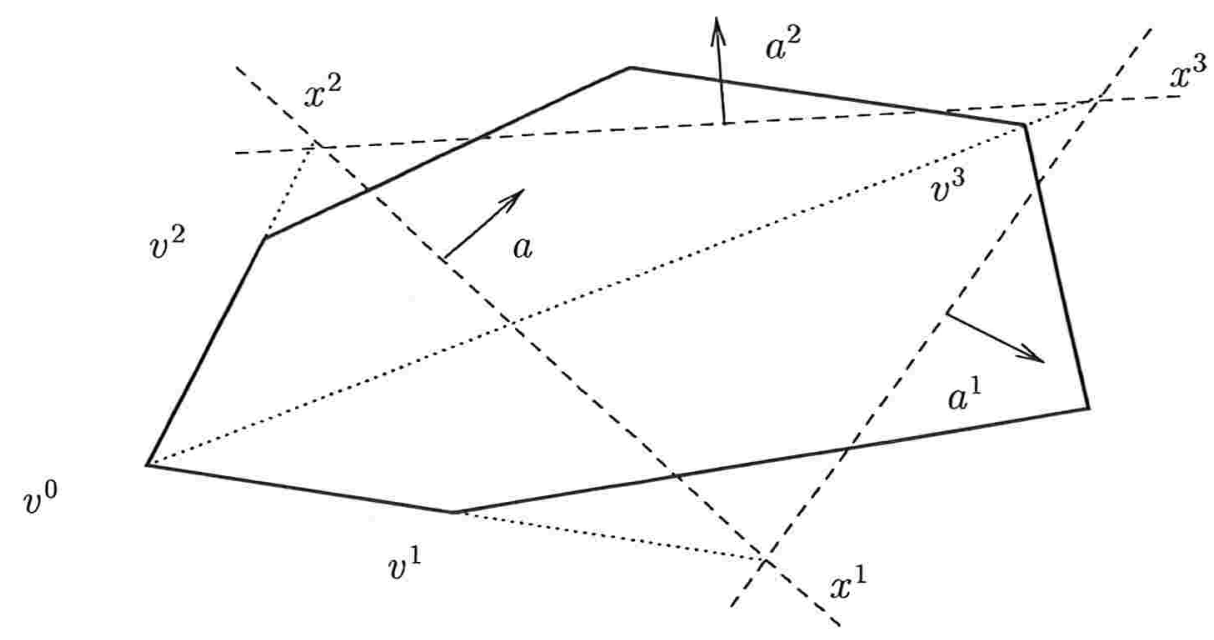

Deste modo uma árvore de subproblemas é criada onde cada nó dá origem a $n$ subproblemas.

Os subproblemas estão todos definidos sobre o mesmo poliedro $X$, pois os cortes de concavidade são descartados logo após a definição dos subproblemas. Isso elimina duas dificuldades do método anterior: a descrição do poliedro não cresce, e basta que o primeiro vértice seja não-degenerado para o algoritmo estar bem-definido. Em contrapartida o processamento pode ficar sobrecarregado com o grande número de subproblemas gerados $\left(n^{p}\right.$ subproblemas de profundidade $\left.p\right)$.

Além disso, foi constatado em [Zwart 1973] que esta estratégia pode ciclar, isto é, um nó associado aos pontos $\left\{x^{i_{1}}, x^{i_{2}}, \ldots, x^{i_{n}}\right\}$ pode descender de um outro nó associado aos mesmos pontos. Muito embora não seja possível construir um tal contra-exemplo em $\mathbb{R}^{2}$, é possível entender o mecanismo utilizado no desenho abaixo. 


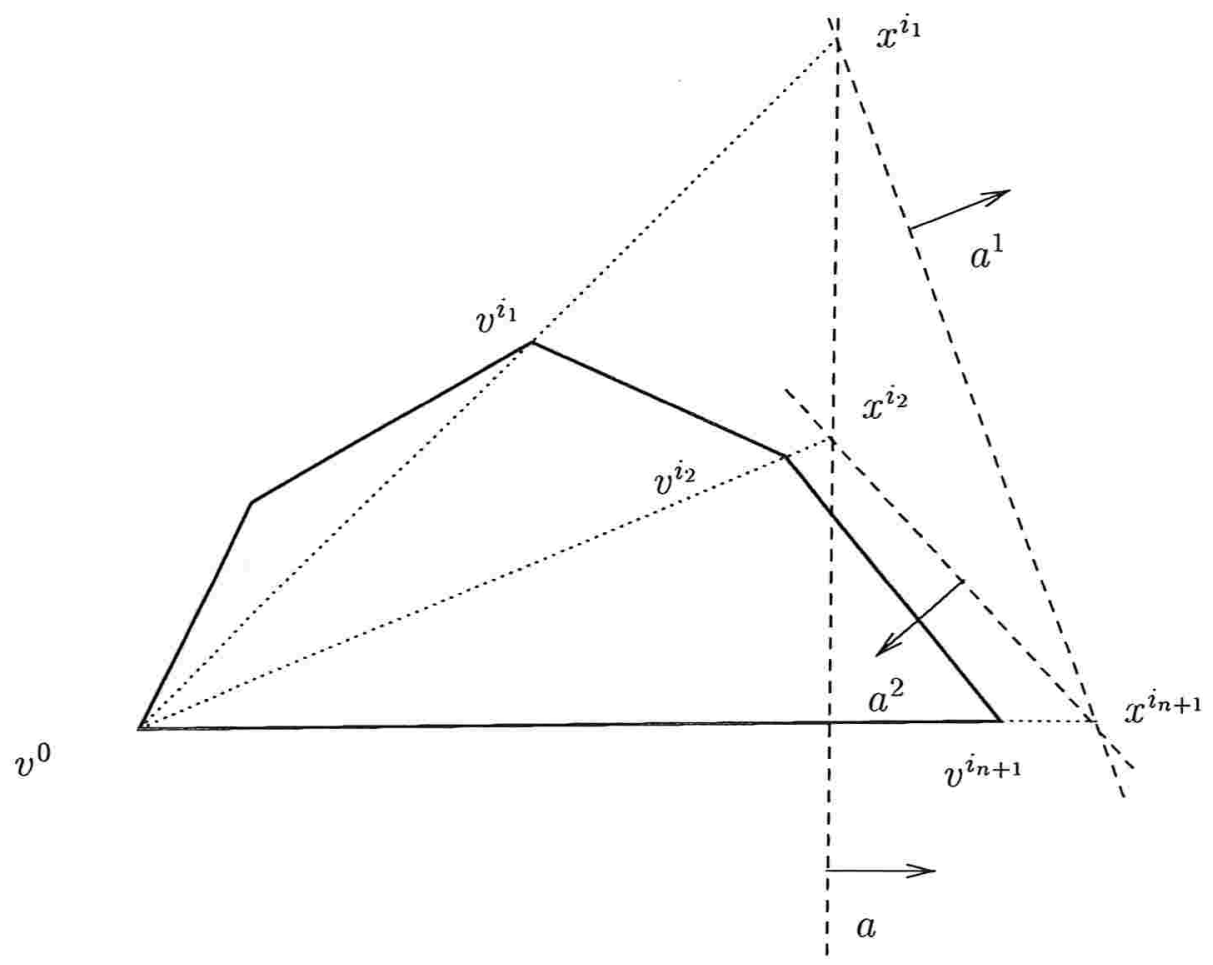

Neste exemplo, o subproblema associado a $\left(x^{i_{1}}, x^{i_{2}}\right)$ dá origem a um subproblema associado a $\left(x^{i_{2}}, x^{i_{n+1}}\right)$, que dá origem por sua vez a um subproblema associado a $\left(x^{i_{2}}, v^{0}\right)$, que por fim dá origem ao subproblema original. O desenho a rigor não está correto porque se $f\left(x^{i_{1}}\right)=f\left(x^{i_{n+1}}\right)=f\left(v^{0}\right)$, então (usando concavidade) a extensão $x^{i_{2}}$ de $v^{i_{2}}$ está sub-estimada. Isso pode ser facilmente corrigido com uma dimensão a mais, garantindo que $x^{i_{1}}, x^{i_{2}}$ e $x^{i_{n+1}}$ não sejam co-lineares.

A propriedade essencial que impediria a ciclagem do método, e que é violada pelo exemplo em [Zwart 1973], é que a solução do PL $\max _{x \in X} a^{t} x$ deveria estar no cone (transladado) gerado por $\left\{v^{i_{1}}-v^{0}, v^{i_{2}}-v^{0}, \ldots, v^{i_{n}}-v^{0}\right\}$. Em geral, se $X \subset \mathbb{R}^{n}$, é necessário adicionar as restrições que definem o cone (vide observação 3.2).

Por curiosidade, a propriedade acima vale "quase" automaticamente em $\mathbb{R}^{2}$ : se a solução $x^{*}$ do PL não está no cone, então $a^{t} x^{*} \leq b$, pois do contrário uma das extensões $x^{i_{1}}$ ou $x^{i_{2}}$ estaria sub-estimada (vide desenho anterior). Esta observação 
pode ser usada para verificar que o método proposto por Tuy sempre funciona em dimensão 2.

O método proposto em [Thoai \& Tuy 1980] relembra este método proposto em [Tuy 1964], porém além de adicionar aos subproblemas as restrições de definição do cone, substitui o $n$-particionamento do cone em cada subproblema pela operação mais simples de bissecção, definida a seguir.

Definição 3.14 (Cones obtidos por bissecção)

Seja $C=$ cone $\left\{v^{i_{1}}-v^{0}, v^{i_{2}}-v^{0}, \ldots, v^{i_{n}}-v^{0}\right\}$, e $r, s \in\{1,2, \ldots, n\}$ tais que

$$
\left\|v^{i_{r}}-v^{i_{s}}\right\|=\max \left\{\left\|v^{i_{j}}-v^{i_{k}}\right\| \mid j, k \in\{1,2, \ldots, n\}\right\}
$$

Seja $\bar{v}=\frac{1}{2} v^{i_{r}}+\frac{1}{2} v^{i_{s}}$. Os cones obtidos por bissecção a partir de C são

$$
\begin{aligned}
& C^{1}=\operatorname{cone}\left\{\bar{v}-v^{0}, v^{i_{k}}-v^{0} \mid k \neq s\right\} e \\
& C^{2}=\operatorname{cone}\left\{\bar{v}-v^{0}, v^{i_{k}}-v^{0} \mid k \neq r\right\} .
\end{aligned}
$$

Note que a operação de bissecção depende da representação do cone através $\operatorname{dos} \operatorname{raios} v^{i_{1}}-v^{0}, v^{i_{2}}-v^{0}, \ldots, v^{i_{n}}-v^{0}$.

\section{Lema 3.15 (Cobertura por bissecção de cones)}

Se $C=\operatorname{cone}\left\{v^{i_{1}}-v^{0}, v^{i_{2}}-v^{0}, \ldots, v^{i_{n}}-v^{0}\right\}$ é tal que $\operatorname{Int}(C) \neq \emptyset$, e os cones $C^{1}$ e $C^{2}$ são obtidos por bissecção a partir de $C$, então $\operatorname{Int}\left(C^{1}\right) \neq \emptyset$, $\operatorname{Int}\left(C^{2}\right) \neq \emptyset$, $\operatorname{Int}\left(C^{1}\right) \cap \operatorname{Int}\left(C^{2}\right)=\emptyset$ e $C=C^{1} \cup C^{2}$.

Prova: Seja $D$ a matriz com colunas $v^{i_{1}}-v^{0}, v^{i_{2}}-v^{0}, \ldots, v^{i_{n}}-v^{0}$. Inicialmente, note que $x \in \operatorname{Int}\left(C^{1}\right)$ se, e somente se, existem $\bar{\mu}>0 \mathrm{e}$ $\mu_{k}>0, k \neq s$ tais que

$$
\begin{aligned}
x & =\bar{\mu}\left(\bar{v}-v^{0}\right)+\sum_{k \neq s} \mu_{k}\left(v^{i_{k}}-v^{0}\right) \\
& =\left(\frac{1}{2} \bar{\mu}+\mu_{r}\right)\left(v^{i_{r}}-v^{0}\right)+\frac{1}{2} \bar{\mu}\left(v^{i_{s}}-v^{0}\right)+\sum_{k \neq r, s} \mu_{k}\left(v^{i_{k}}-v^{0}\right) .
\end{aligned}
$$


Isso garante que $D^{-1} x>0$ e, além disso, $D_{r}^{-1} x=\frac{1}{2} \bar{\mu}+\mu_{r}>\frac{1}{2} \bar{\mu}=D_{s}^{-1} x$. Por outro lado, se $x=\bar{\mu}\left(\bar{v}-v^{0}\right)+\sum_{k \neq s} \mu_{k}\left(v^{i_{k}}-v^{0}\right)$ satisfaz $D^{-1} x>0$ e $D_{r}^{-1} x>D_{s}^{-1} x$, então $\bar{\mu}>0$ e $\mu_{k}>0, k \neq s$, e assim $x \in \operatorname{Int}\left(C^{1}\right)$. Isso mostra que

$$
\operatorname{Int}\left(C^{1}\right)=\left\{x \in \operatorname{Int}(C) \mid D_{r}^{-1} x>D_{s}^{-1} x\right\} .
$$

De maneira análoga mostra-se que

$$
\operatorname{Int}\left(C^{2}\right)=\left\{x \in \operatorname{Int}(C) \mid D_{r}^{-1} x<D_{s}^{-1} x\right\} .
$$

De posse desta caracterização, tem-se trivialmente

$$
\operatorname{Int}\left(C^{1}\right) \cap \operatorname{Int}\left(C^{2}\right)=\emptyset
$$

e, além disso,

$$
\begin{aligned}
C^{1} \cup C^{2} & =\overline{\operatorname{Int}\left(C^{1}\right)} \cup \overline{\overline{\operatorname{Int}\left(C^{2}\right)}} \\
& =\left\{x \in C \mid D_{r}^{-1} x \geq D_{s}^{-1} x \text { ou } D_{r}^{-1} x \leq D_{s}^{-1} x\right\} \\
& =C .
\end{aligned}
$$

Por fim, considere $x=\left(v^{i_{r}}-v^{0}\right)+\sum_{k=1}^{n}\left(v^{i_{k}}-v^{0}\right)$. É claro que $D_{r}^{-1} x=2$ e $D_{k}^{-1} x=1, \forall k \neq r$ e, deste modo, $x \in \operatorname{Int}\left(C^{1}\right)$. Analogamente, obtém-se $\operatorname{Int}\left(C^{2}\right) \neq \emptyset$.

\section{Método de Thoai \& Tuy (bissecção de cones)}

0. Seja $v^{0}$ um mínimo local-* de $f(\cdot)$ em $X$, e sejam $v^{1}, v^{2}, \ldots, v^{n}$ seus vizinhos. Faça $v^{*}=v^{0}$ e $f^{*}=f\left(v^{0}\right)$.

Faça $C^{i} \leftarrow \emptyset$ e $d_{i} \leftarrow 0$, para todo $i \in \mathbb{N}$.

Faça $C^{1} \leftarrow \operatorname{cone}\left\{v^{1}, v^{2}, \ldots, v^{n}\right\}, k \leftarrow 1$ e $\bar{k} \leftarrow 1$.

1. Enquanto $C^{k}=\emptyset$, faça $k \leftarrow k+1$. 
Com posse deste resultado, conclui-se que o NCP

$$
\left\{\begin{array}{l}
x \geq 0 \\
F(x) \geq 0 \\
x^{t} F(x)=0
\end{array}\right.
$$

com $\nabla F(x)$ simétrico para todo $x$ é equivalente às condições de otimalidade de primeira ordem do problema de otimização

$$
\begin{cases}\min & f(x)=\int_{0}^{1} F(s x)^{t} x d s \\ \text { s.a. } & x \geq 0\end{cases}
$$

No caso em questão, parece difícil incorporar a restrição $\mathbb{1}^{t} x=1$ (ou $x \neq 0$ ) sem quebrar a simetria do problema. Pode-se constatar esta dificuldade ao considerar-se o NCP equivalente, obtido ao acrescentar uma variável auxiliar $\alpha$ satisfazendo

$$
\left\{\begin{array}{l}
w=(B-\mu A) x \\
\alpha=-1+\mathbb{1}^{t} x \\
w \geq 0 \\
x \geq 0 \\
w^{t} x=\alpha \mu=0
\end{array}\right.
$$

onde $\mu=\frac{1}{\lambda}$ em relação ao problema original. Neste contexto, as hipóteses do teorema clássico não se verificam.

A partir da reformulação do (EiCP) como um problema de Programação NãoLinear várias conclusões podem ser tiradas. A primeira delas é uma caracterização da viabilidade do (EiCP).

Proposição 2.21 (Viabilidade do (EiCP) simétrico)

$O$ (EiCP) simétrico é viável se, e somente se, existe $x \geq 0$ tal que $x^{t} A x>0$. 
Prova : Suponha, por um lado, que o (EiCP) é viável. Então, se $(\lambda, x)$ é uma solução viável qualquer, tem-se $x^{t} A x=\lambda x^{t} B x>0$, pois $x \neq 0$ e $B$ é positiva definida.

Suponha, por outro lado, que exista um $\hat{x} \geq 0$ tal que $\hat{x}^{t} A \hat{x}>0$. Como $\lambda(x)$ é uma função contínua (em $x \neq 0$ ) e o conjunto $X=\left\{x \geq 0 \mid \mathbb{1}^{t} x=1\right\}$ é compacto, existe um ponto $\bar{x} \in X$ tal que $\lambda(\bar{x}) \geq \lambda(x), \forall x \in X$. Este ponto satisfaz as condições de equilíbrio do (OEiCP) e, em particular, satisfaz $\lambda(\bar{x}) \geq \lambda(\hat{x})>0$. Portanto, $\bar{x}$ é uma solução do (EiCP).

Para a solução prática do (EiCP) simétrico, qualquer método de subida de gradiente, a partir de uma solução inicial $\hat{x} \in X$ tal que $\hat{x}^{t} A \hat{x}>0$, obtém um ponto de equilíbrio que é solução do problema. A não-convexidade da função objetivo não é realmente um problema, visto que o problema de otimização global (OEiCP) não precisa ser resolvido.

Algumas classes de matrizes $(A)$ que possuem uma tal solução inicial podem ser facilmente identificadas, por exemplo, matrizes copositivas não-nulas e, em particular, matrizes semi-definidas positivas não-nulas. Em geral, encontrar um tal ponto inicial é um problema NP-completo (proposição 2.14). Mas para uma classe muito grande de matrizes este problema é trivial, como mostra a proposição a seguir. Para estas matrizes, um ponto inicial pode ser obtido por inspeção, e a solução do (EiCP) é obtida facilmente a partir daí. 
2. Se $k>\bar{k}$, pare. $v^{*}$ é uma solução ótima com valor ótimo $f^{*}$. Caso contrário, seja $C^{k}=\operatorname{cone}\left\{v^{i_{1}}-v^{0}, v^{i_{2}}-v^{0}, \ldots, v^{i_{n}}-v^{0}\right\}$, e $d_{k}=\max \left\{\left\|v^{i_{r}}-v^{i_{s}}\right\| \mid r, s=1,2, \ldots, n, r \neq s\right\}$.

3. Compute, para cada $j=1,2, \ldots, n$,

$$
\theta_{j}=\sup \left\{\theta \mid f\left(v^{0}+\theta\left(v^{i_{j}}-v^{0}\right)\right) \geq f^{*}\right\}
$$

Se $I=\left\{j \mid \theta_{j}<\infty\right\}=\emptyset$, faça $k \leftarrow k+1$ e volte ao passo 1 .

4. Calcule o corte de concavidade $a^{t} x \geq b$ da definição 3.7 , e seja $x^{*}$ a solução ótima de $\max _{x \in X \cap C^{k}} a^{t} x$. Se $a^{t} x^{*} \leq b$, faça $k \leftarrow k+1$ e volte ao passo 1 . Se $f\left(x^{*}\right)<f^{*}$, faça $v^{*}=x^{*}$ e $f^{*}=f\left(x^{*}\right)$.

5. Sejam $C^{2 k}$ e $C^{2 k+1}$ os cones obtidos por bissecção a partir de $C^{k}$. Faça $\bar{k} \leftarrow 2 k+1, k \leftarrow k+1$, e volte ao passo 1 .

Note que a árvore de enumeração dos cones foi propositalmente organizada como

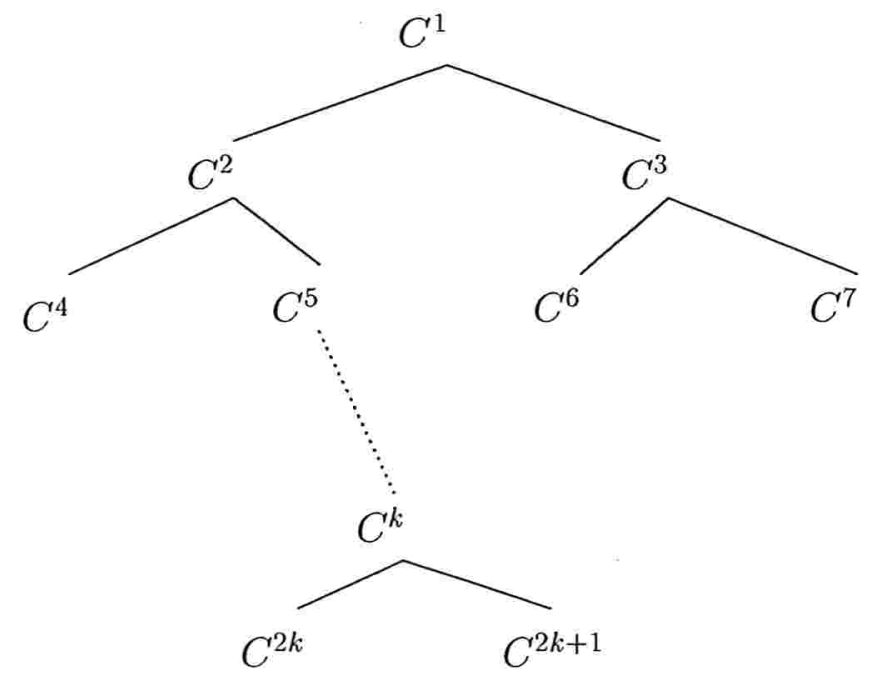




\section{Lema 3.16 (Cobertura por cones no Método de Thoai \& Tuy)}

Seja $C^{k}$ a seqüência produzida pelo método de Thoai \& Tuy. Então, para todo $p \in \mathbb{N}$,

$$
\begin{gathered}
C^{1} \supseteq \bigcup_{i=2^{p}}^{2^{p+1}-1} C^{i}, e \\
\operatorname{Int}\left(C^{2^{p}+i}\right) \cap \operatorname{Int}\left(C^{2^{p}+j}\right)=\emptyset, \forall i, j=0, \ldots, 2^{p}-1, i \neq j .
\end{gathered}
$$

Prova: Considerando-se que o método não aplica bissecção a alguns cones $C^{k}$, quando então $C^{2 k}=C^{2 k+1}=\emptyset$, o lema 3.15 traduz-se por $C^{k} \supseteq C^{2 k} \cup C^{2 k+1}$ e $\operatorname{Int}\left(C^{2 k}\right) \cap \operatorname{Int}\left(C^{2 k+1}\right)=\emptyset$. O resultado é obtido pela aplicação destas propriedades $p$ vezes.

\section{Lema 3.17 (Convergência de cones para subseqüências encaixadas)}

Seja $C^{k_{1}} \supset C^{k_{2}} \supset \cdots C^{k_{j}} \supset \cdots$ uma subseqüência de cones tal que $\operatorname{Int}\left(C^{k_{j}}\right) \neq \emptyset e$ $C^{k_{j+1}}$ é obtido por bisseç̧ão a partir de $C^{k_{j}}$, para todo $j \in \mathbb{N}$. Então

$$
d_{k_{j+\frac{n(n-1)}{2}}} \leq \frac{d_{k_{j}}}{2}
$$

e, em particular, $\lim _{j \rightarrow \infty} d_{k_{j}}=0$.

Prova : Sejam $\left(\alpha_{1}^{j}, \alpha_{2}^{j}, \ldots, \alpha_{\frac{n(n-1)}{2}}^{j}\right)$ todos os valores de $\left\|v^{r}\left(k_{j}\right)-v^{s}\left(k_{j}\right)\right\|$, para $r, s=1,2, \ldots, n, r \neq s$, ordenados como a seguir

$$
\alpha_{1}^{j} \geq \alpha_{2}^{j} \geq \ldots \geq \alpha_{\frac{n(n-1)}{2}}^{j}
$$

onde $\left\{v^{i}\left(k_{j}\right)-v^{0} \mid i=1,2, \ldots, n\right\}$ são os raios que definem o cone $C^{k_{j}}$. Note que a desigualdade $\alpha_{i}^{j} \geq \alpha_{i}^{j+m}$ é satisfeita por construção. Prova-se a seguir que $\alpha_{1}^{j+\frac{n(n-1)}{2}} \leq \frac{\alpha_{1}^{j}}{2}$ e isso prova o resultado (pois $d_{k_{j}}=\alpha_{1}^{j}, \forall j \in \mathbb{N}$ ). Suponha, por contradição, que $\alpha_{1}^{j+\frac{n(n-1)}{2}}>\frac{\alpha_{1}^{j}}{2}$. Pela construção do processo de bissecção, para cada $m=0,1, \ldots, \frac{n(n-1)}{2}$ existe um índice máximo $i(m)$ tal que $\alpha_{1}^{j+\frac{n(n-1)}{2}}=\alpha_{i(m)}^{j+m}$. Mais ainda, os números 
$\left(\alpha_{1}^{j+m}, \alpha_{2}^{j+m}, \ldots, \alpha_{\frac{n(n-1)}{2}}^{j+m}\right)$ e $\left(\alpha_{1}^{j+m+1}, \alpha_{2}^{j+m+1}, \ldots, \alpha_{\frac{n(n-1)}{2}}^{j+m+1}\right)$ são os mesmos com a única exceção de que $\alpha_{1}^{j+m}$ aparece dividido por 2 em $\alpha^{j+m+1}$.

Como, além disso,

$$
\alpha_{i(m+1)}^{j+m+1}=\alpha_{1}^{j+\frac{n(n-1)}{2}}>\frac{\alpha_{1}^{j}}{2} \geq \frac{\alpha_{1}^{j+m}}{2}
$$

e $i(m)$ é o maior índice tal que $\alpha_{1}^{j+\frac{n(n-1)}{2}}=\alpha_{i(m)}^{j+m}$, tem-se que

$$
i(m+1)<i(m), m=0,1, \ldots, \frac{n(n-1)}{2} .
$$

Mas como $i(m) \in\left\{0,1, \ldots, \frac{n(n-1)}{2}\right\}$ tem-se, obrigatoriamente,

$$
i(m)=\frac{n(n-1)}{2}-m
$$

onde $i\left(\frac{n(n-1)}{2}\right)=0$ indica que

$$
\alpha_{1}^{j+\frac{n(n-1)}{2}} \leq \frac{\alpha_{1}^{j}}{2}
$$

Esta contradição conclui a demonstração.

O resultado do lema 3.17 aqui apresentado é uma versão mais forte de um resultado de [Thoai \& Tuy 1980], e além disso possui uma demonstração mais simples do que aquele. Em [Thoai \& Tuy 1980] se estabelece apenas $\lim _{j \rightarrow \infty} d_{k_{j}}=0$ para uma subseqüência $\left\{C^{k_{j}}\right\}$ de cones encaixados. A propriedade adicional

$$
d_{k+\frac{n(n-1)}{2}} \leq \frac{d_{k_{j}}}{2}
$$

garante a convergência de $d_{k}$ para toda a seqüência $\left\{C^{k}\right\}$ gerada pelo algoritmo. Este resultado não foi estabelecido em [Thoai \& Tuy 1980].

Proposição 3.18 (Convergência de $d_{k}$ no Método de Thoai \& Tuy) Sejam $C^{k}$ e $d_{k}$ as seqüências produzidas pelo método de Thoai \& Tuy. Então $\lim _{k \rightarrow \infty} d_{k}=0$ 
Prova : Por construção, tem-se que

$$
C^{k} \supseteq \bigcup_{i=0}^{2^{p} k-1} C^{2^{p} k+i}
$$

onde os cones $C^{2^{p} k+i}$ acima são obtidos através de $p$ bissecções a partir de $C^{k}$. Portanto

$$
d_{2^{p} k+i} \leq d_{k}, \forall i=0,1, \ldots, 2^{p} k-1
$$

$\mathrm{e}$, além disso, se $\bar{p}=\frac{n(n+1)}{2}$,

$$
d_{2^{\bar{p}} k+i} \leq \frac{d_{k}}{2}, \forall i=0,1, \ldots, 2^{\bar{p}} k-1
$$

Com isso,

$$
d_{k} \leq \frac{1}{2^{m}} d_{1}, \forall k=2^{m \bar{p}}, 2^{m \bar{p}}+1, \ldots, 2^{(m+1) \bar{p}}-1
$$

e portanto $\lim _{k \rightarrow \infty} d_{k}=0$.

\section{Proposição 3.19 (Convergência do Método de Thoai \& Tuy)}

Se o método de Thoai 8 Tuy termina em tempo finito, $v^{*}$ é uma solução ótima de $(P P C)$ com valor ótimo $f^{*}$. Do contrário, os valores de $v^{*}$ e $f^{*}$ convergem, em tempo finito, para uma solução ótima de (PPC), e toda subseqüência de cones encaixados não-vazios $C^{k_{1}} \supset C^{k_{2}} \supset \cdots C^{k_{j}} \supset \cdots$ converge para um raio $r^{k}=\bigcap_{i=1}^{\infty} C^{k_{i}}$ contendo um vértice $v \neq v^{0}$ de $X$ tal que $f(v)=f^{*}$.

Prova: O único critério de parada do algoritmo é $k>\bar{k}$, onde $\vec{k}$ é o maior índice associado aos cones gerados por bissecção no algoritmo. Se o algoritmo termina em tempo finito, $f^{*} \leq \min _{x \in X \cap C^{k}} f(x)$ para todo $k \leq \bar{k}$, e como $X \subset \cup_{k \leq k} C^{k}$, tem-se que $f^{*} \leq \min _{x \in X} f(x)$. Mas $f^{*}=f\left(v^{*}\right)$ onde $v^{*} \in X$, e portanto $f^{*}=\min _{x \in X} f(x)$. 
Por outro lado, se o algoritmo não termina, pela proposição 3.18 tem-se $\lim _{k \rightarrow \infty} d_{k}=0$. Seja $\varepsilon>0$ tal que qualquer cone de diâmetro $d_{k} \leq \varepsilon$ gerado pelo algoritmo contém no máximo um vértice de $X$ (além de $v^{0}$ ). Pelo lema 3.17 existe um $\bar{m}$ tal que $d_{k} \leq \varepsilon$ para $k=2^{\bar{m}}, 2^{\bar{m}}+1, \ldots, 2^{\bar{m}+1}-1$ e como $X \subset \bigcup_{k=1}^{2^{m+1}-1} C^{k}$, um vértice ótimo será encontrado até a iteração $k=2^{\bar{m}+1}-1$.

Se $C^{k_{1}} \supset C^{k_{2}} \supset \cdots C^{k_{j}} \supset \cdots$ é tal que $r^{k}=\bigcap_{i=1}^{\infty} C^{k_{i}} \neq \emptyset$, então $r^{k}$ é um raio porque $\lim _{j \rightarrow \infty} d_{k_{j}}=0$ (lema 3.17). O raio $r^{k}$ contém um vértice $v \neq v^{0}$ pela proposição 3.12 e pelo critério de interrupção de bissecção do passo 4 do método. Além disso, pelo critério de interrupção de bissecção do passo $3, I \neq \emptyset$ para todos os cones $C^{k_{j}}$. Seja $x^{i}\left(k_{j}\right)$ uma das extensões tais que $i \in I$, para cada $j \in \mathbb{N}$. Então $\lim _{j \rightarrow \infty}\left\|v-x^{i}\left(k_{j}\right)\right\|=0$, e pela continuidade da $f(\cdot)$, tem-se $f(v)=\lim _{j \rightarrow \infty} f\left(x^{i}\left(k_{j}\right)\right)=f^{*}$.

Note portanto que o número de subseqüências distintas de cones encaixados não-vazios é limitado pelo número de vértices ótimos do poliedro original $X$.

Apesar do resultado de convergência deste algoritmo, o bom funcionamento dele depende também de uma certa quantidade de sorte. O processo de subdivisão de cones pode demorar muito até produzir um cone contendo a solução ótima, e de tal forma que ela seja a solução de $\max _{x \in X \cap C^{k}} a^{t} x$, onde $a^{t} x \geq b$ é o corte de Tuy produzido. Além disso, a solução ótima poderia ser encontrada logo no início do algoritmo, e no entanto o processo de exaustão dos cones demorar muito.

Um exemplo poderá ilustrar a dificuldade. Suponha que $C^{1}=$ cone $\left\{v^{1}-v^{0}, v^{2}-v^{0}, \ldots, v^{n}-v^{0}\right\}$, e que

$$
\left\|v^{r}-v^{s}\right\|=1, \forall r, s=1,2, \ldots, n, r \neq s .
$$


Pelo processo de bissecção, é fácil ver que

$$
d_{k}=1, \forall k=1,2, \ldots, 2^{\frac{n(n+1)}{2}}-1
$$

e, em geral (vide demonstração da proposição 3.18),

$$
d_{k}=\frac{1}{2^{m}}, \forall k=2^{\frac{m(n+1)}{2}}, 2^{m \frac{n(n+1)}{2}}+1, \ldots, 2^{(m+1) \frac{n(n+1)}{2}}-1,
$$

ou seja, o número de cones $C^{k}$ para os quais $d_{k}$ permanece constante $=\frac{1}{2^{m}}$ é dado por

$$
\left(2^{\frac{n(n+1)}{2}}\right)^{m}
$$

Em $\mathbb{R}^{50}$, por exemplo, este número é próximo de $\left(10^{384}\right)^{m}$, ou seja, a técnica de bissecção de cones precisa gerar aproximadamente $10^{7650}$ subcones até que o diâmetro de todos eles seja limitado por $10^{-6}$.

Esta "certa quantidade de sorte" pode ser favorecida através do uso de heurísticas nos subproblemas. Por exemplo, ao resolver-se um subproblema linear $\max _{x \in X \cap C^{k}} a^{t} x$ os valores de $f(v)$ nos vértices enumerados podem ser comparados com $f^{*}$, com custo adicional muito baixo. Além disso, um número maior de mínimos locais-* podem ser procurados em cada subcone, a partir de vértices iniciais distintos.

\subsection{Os Métodos de Tuy e Thoai \& Tuy Para o LCP}

Os métodos da seção anterior podem ser diretamente aplicados ao Problema de Complementaridade Linear, se $X=\{z \geq 0 \mid w(z)=M z+q \geq 0\}$ é limitado e $\operatorname{Int}(X) \neq \emptyset$.

Definição 3.20 (Problema de Complementaridade Linear - LCP)

$$
(L C P)\left\{\begin{aligned}
z & \geq 0 \\
w(z)=M z+q & \geq 0 \\
z^{t} w(z) & =0
\end{aligned}\right.
$$


Isso é feito através da reformulação do (LCP) como um Problema de Programação Côncava [Thoai \& Tuy 1983].

Definição 3.21 (LCP como Problema de Programação Côncava)

$$
(L C P-P P C) \begin{cases}\min & f(z)=\sum_{i=1}^{n} \min \left\{z_{i}, w_{i}(z)\right\} \\ & z \in X .\end{cases}
$$

Note que a função objetivo $f(\cdot)$ é côncava porque é a soma de mínimos de funções lineares, e satisfaz $f(z) \geq 0$ para todo $z \in X$. A equivalência dos dois problemas é trivial.

Proposição 3.22 (Equivalência de LCP e LCP-PPC)

$z$ é solução do (LCP) se, e somente se, z é solução ótima do (LCP-PPC) com $f(z)=0$.

Prova : Basta notar que se $z \in X$, então $z \geq 0$ e $w(z) \geq 0$, logo

$$
w^{t} z=0 \Longleftrightarrow w_{i} z_{i}=0, \forall i \Longleftrightarrow f(z, w)=0
$$

Em [Thoai \& Tuy 1983] o método para programação côncava proposto em [Thoai \& Tuy 1980] é adaptado ao LCP, na tentativa de lidar inclusive com LCPs associados a poliedros ilimitados. Mostra-se que se o algoritmo gera uma seqüência infinita de cones $C^{k}$ tais que $X \cap C^{k}$ é ilimitado, então o (LCP) é inviável. Apesar desta caracterização, nota-se claramente que o algoritmo não é capaz de verificar que um (LCP) é inviável. Por esta razão, mantém-se a hipótese de limitação do poliedro no presente contexto; além disto, esta hipótese é essencial ao método de 
[Tuy 1964], que utiliza somente cortes de concavidade. Mostra-se a seguir que a hipótese de $X=\{z \geq 0 \mid w(z)=M z+q \geq 0\} \neq \emptyset$ ser um poliedro limitado depende exclusivamente da matriz $M$.

\section{Lema 3.23 (Classe de matrizes associadas a poliedros limitados)}

$X=\{z \geq 0 \mid w(z)=M z+q \geq 0\} \neq \emptyset$ é limitado se, e somente se, $M$ não é uma matriz $S_{0}$, ou seja, se

$$
\left.\begin{array}{r}
h \geq 0 \\
M h \geq 0
\end{array}\right\} \Longrightarrow h=0 .
$$

Prova : Basta ver que $X \neq \emptyset$ é limitado se, e somente se, o cone recessional $C=\{h \geq 0 \mid M h \geq 0\}$ satisfaz $C \equiv\{0\}$. Lembrando a definição da classe $S_{0}$ como a classe das matrizes $M$ tais que

$$
\exists h \neq 0 \text { tal que }\left\{\begin{array}{l}
h \geq 0 \\
M h \geq 0
\end{array}\right.
$$

tem-se o resultado.

A classe de matrizes $S_{0}$ contém a classe de matrizes semi-monótonas $E_{0}$, que por sua vez contém as classes de matrizes $K, P_{0}$, Positivas Semi-Definidas, Copositivas, Não-Negativas, Diagonal Dominantes, Adequadas e Regulares (vide glossário e [Cottle, Pang \& Stone 1992]). Deste modo, $S_{0}$ contém todas as classes de matrizes para as quais se conhece algoritmos eficientes para processar o (LCP) correspondente [Cottle, Pang \& Stone 1992, Murty 1988].

A classe Não- $S_{0}$ das matrizes associadas a poliedros limitados é subclasse da classe $R_{0}$ das matrizes Pseudo-Regulares, definida pela condição

$$
\left.\begin{array}{rl}
z & \geq 0 \\
M z & \geq 0 \\
z^{t} M z & =0
\end{array}\right\} \Longrightarrow z=0 .
$$


A inclusão Não- $S_{0} \subseteq R_{0}$ é trivial. $R_{0}$ é a classe das matrizes para as quais o (LCP) homogêneo (i.e., com $q=0$ ) possui somente a solução trivial. Outra caracterização da classe $R_{0}$ é como a classe de matrizes tais que o conjunto de soluções do (LCP) é limitado [Cottle, Pang \& Stone 1992].

A estrutura do problema (LCP-PPC) pode ser explorada a fim de acelerar os métodos apresentados na seção anterior.

Por exemplo, o valor $f^{*}$ utilizado nestes métodos armazena o valor da melhor solução viável do (PPC) já encontrada. Estes valores fornecem estimativas cada vez melhores do ótimo global, e fornecem alguma informação útil na hipótese do método atingir o número limite de iterações. No caso do (LCP-PPC) não interessa obter soluções com $f^{*}>0$, porque nenhuma delas é solução do (LCP). Assim, na geração de cortes de concavidade para o (LCP-PPC) pode-se adotar $f^{*}=0$ na definição dos valores $\theta_{i}$. Inicializa-se $v^{*}$ com um ponto inviável $v^{-1}$ arbitrário, e para todas as soluções $x$ dos subproblemas testa-se a validade de $f(x)=0$. Se uma solução com $f(x)=0$ é encontrada, o método pára fornecendo esta solução. Se um dos critérios de parada é atingido e $v^{*}=v^{-1}$, então o problema não tem solução.

\section{Lema 3.24 (Corretude do corte de Tuy para o (LCP))}

Seja $v^{0}$ um mínimo local- * de $f(\cdot)$ em $X$ tal que $f\left(v^{0}\right)>0$, e seja $a^{t} x \geq b$ o corte de concavidade gerado a partir de um cone $C=\operatorname{cone}\left\{x^{1}-v^{0}, x^{2}-v^{0}, \ldots, x^{n}-v^{0}\right\}$ com $f^{*}=0$. Então, para todo $x \in X \cap C$ tal que $a^{t} x<b$, tem-se $f(x)>0$. Em outras palavras, o corte $a^{t} x \geq b$ não elimina nenhuma solução do (LCP) em $X \cap C$.

Prova : Se $x \in X \cap C$ e $a^{t} x<b$, então pelo lema $3.8, x$ escreve-se como $x=\bar{x}+h$, onde

$$
\begin{aligned}
& \bar{x} \in \operatorname{conv}\left\{v^{0}, x^{i} \mid i \in I\right\}, \\
& h \in \operatorname{cone}\left\{x^{i}-v^{0} \mid i \notin I\right\},
\end{aligned}
$$


e $I$ e $x^{1}, x^{2}, \ldots, x^{n}$ são como na definição 3.7. Mais ainda, $\bar{x}=\alpha_{0} v^{0}+\sum_{i \in I} \alpha_{i} x^{i} \operatorname{com} \alpha_{0}>0$ (pois $\left.a^{t} x<b\right)$, assim

$$
f(\bar{x}) \geq \alpha_{0} f\left(v^{0}\right)+\sum_{i \in I} \alpha_{i} f\left(x^{i}\right)>0
$$

e, pelo lema $3.9, f(x) \geq f(\bar{x})>0$.

\section{Proposição 3.25 (Métodos de Tuy e Thoai \& Tuy com $f^{*}=0$ )}

Os métodos de Tuy e.Thoai \& Tuy para o (LCP-PPC), inicializados com $f^{*}=0$ e adicionados do teste $f(x)=0$ para todos os pontos intermediários gerados, possuem os mesmos resultados de corretude e convergência das proposições 3.13 e 3.19. Mais ainda, o método de Thoai \& Tuy de particionamento de cones sempre termina em tempo finito.

Prova : Pelo lema anterior, os cortes de concavidade em um cone $C$ com $f^{*}=0$ não cortam nenhuma solução do (LCP) em $X \cap C$, e isso garante a corretude dos cortes gerados. Tem-se então os resultados de corretude e convergência das proposições 3.13 e 3.19 .

A convergência do método de Thoai \& Tuy em tempo finito para LCPs com solução foi estabelecida em 3.19. Se, por outro lado, o (LCP) não possui solução, então existe um $\varepsilon>0$ tal que $f(x) \geq \varepsilon, \forall x \in X$. Pela proposição 3.19 , uma subseqüência de cones encaixados $C^{k_{1}} \supset C^{k_{2}} \supset \cdots C^{k_{j}} \supset \cdots$ não-vazios deveria conter um vértice $v \in X$ tal que $f(v)=f^{*}=0$, o que é impossível. Portanto não pode existir uma tal subseqüência, e o método termina em tempo finito.

Outro momento onde a estrutura do (LCP-PPC) pode ser utilizada a favor dos métodos de [Tuy 1964, Thoai \& Tuy 1983] é no cômputo dos valores $\theta_{j}$ e das 
extensões $x^{j}$. Como $f(z)=\sum_{i=1}^{n} \min \left\{z_{i}, w_{i}(z)\right\}$ e $w(z)$ é uma função afim, tem-se que $f(\cdot)$ é uma função côncava linear por trechos, bem como, em particular, a função $f^{j}(\theta)=f\left(v^{0}+\theta\left(v^{j}-v^{0}\right)\right)$.

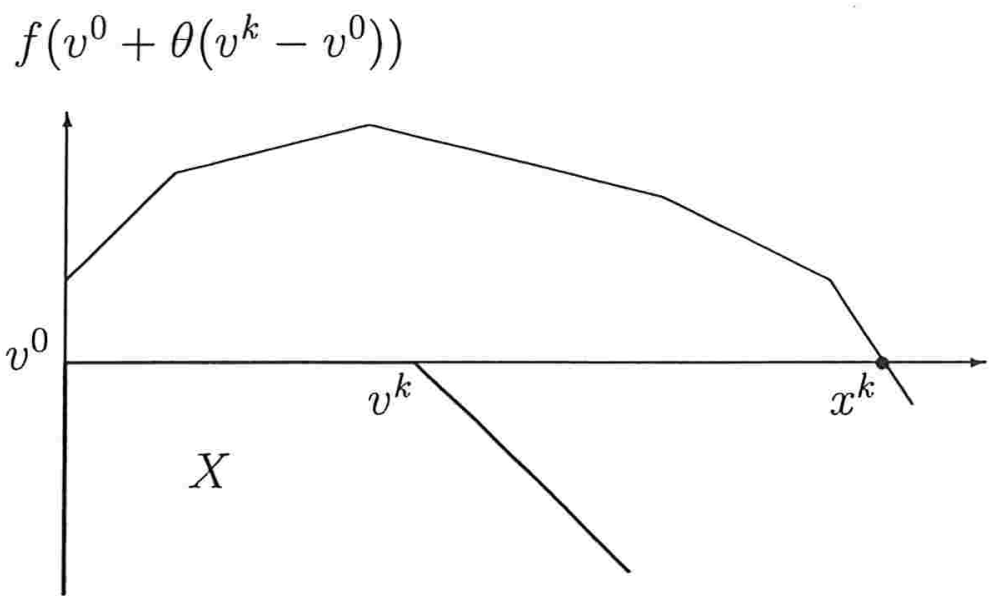

Lema 3.26 (Número de quebras de $f^{j}(\theta)=\left(v^{0}+\theta\left(v^{j}-v^{0}\right)\right)$ )

A função $f^{j}(\theta)=f\left(v^{0}+\theta\left(v^{j}-v^{0}\right)\right)$, para $\theta \geq 0$ possui no máximo $n$ quebras, que definem até $n+1$ trechos lineares.

Prova : Seja $y^{j}(\theta)=v^{0}+\theta\left(v^{j}-v^{0}\right)$. Então as quebras de $f^{j}(\theta)=f\left(y^{j}(\theta)\right)$ correspondem aos valores de $\theta$ para os quais as parcelas $y_{i}^{j}-w_{i}\left(y^{j}\right)$ trocam de sinal; como a direção $v^{j}-v^{0}$ é constante, cada parcela troca de sinal no máximo uma vez, e portanto as quebras ocorrem no máximo $n$ vezes.

Assim, cada $\theta_{j}$ pode ser construído em tempo $\mathcal{O}(n)$ pelo seguinte algoritmo.

Construção de $x^{j}$ e $\theta_{j}$ para o (LCP-PPC)

0. Faça $\theta_{j}=1$ e $x^{j}=v^{j}$. Defina $\Delta^{z}=v^{j}-v^{0}$ e $\Delta^{w}=w\left(v^{j}\right)-w\left(v^{0}\right)$. 
1. Se $f\left(x^{j}\right)=0$ pare. $x^{j}$ e $\theta_{j}$ correspondem à extensão procurada.

2. Compute, se possível, $\bar{i}=\operatorname{argmin}\left\{\left|\frac{x_{\bar{i}_{1}}^{j}-w_{\bar{i}_{1}}\left(x^{j}\right)}{\Delta \Delta_{\bar{i}_{1}}^{z}-\Delta_{\bar{i}_{1}}^{w}}\right|,\left|\frac{x_{\bar{i}_{2}}^{j}-w_{\bar{i}_{2}}\left(x^{j}\right)}{\Delta{\overline{\bar{i}_{2}}}_{2}-\Delta_{\bar{i}_{2}}^{w}}\right|\right\}$, onde

$$
\left.\begin{array}{l}
\bar{i}_{1}=\operatorname{argmin}\left\{\begin{array} { l } 
{ | \frac { x _ { i } ^ { j } - w _ { i } ( x ^ { j } ) } { \Delta _ { i } ^ { z } - \Delta _ { i } ^ { w } } | } \\
{ \overline { i } _ { 2 } = \operatorname { a r g m i n } }
\end{array} \left\{\begin{array}{l}
x_{i}^{j}-w_{i}\left(x^{j}\right)>0 \text { e } \Delta_{i}^{z}-\Delta_{i}^{w}<0 \\
\Delta_{i}^{j}-w_{i}\left(x_{i}^{j}\right) \\
\Delta_{i}^{z}
\end{array} \mid x_{i}^{j}-w_{i}\left(x^{j}\right)<0 \text { e } \Delta_{i}^{z}-\Delta_{i}^{w}>0\right.\right.
\end{array}\right\} \text { e }
$$

e faça $\Delta^{\theta}=\left|\frac{x_{\bar{i}}^{j}-w_{\bar{i}}\left(x^{j}\right)}{\Delta_{\bar{i}}^{z}-\Delta_{\bar{i}}^{w}}\right|$. Se não for possível computar $\bar{i}$, vá para o passo 5 .

3. Faça $y^{j}=x^{j}+\Delta^{\theta}\left(v^{j}-v^{0}\right)$. Se $f\left(y^{j}\right) \geq 0$, faça $x^{j}=y^{j}, \theta_{j}=\theta_{j}+\Delta^{\theta}$ e volte para o passo 1 .

4. Como $f\left(x^{j}\right)>0, f\left(y^{j}\right)<0$, e não há quebras entre $x^{j}$ e $y^{j}$, a função $f(\cdot)$ é linear neste trecho. Portanto faça

$$
\begin{gathered}
\theta_{j}=\theta_{j}+f\left(x^{j}\right) \frac{\Delta^{\theta}}{f\left(x^{j}\right)-f\left(y^{j}\right)}, \\
x^{j}=v^{0}+\theta_{j}\left(v^{j}-v^{0}\right)
\end{gathered}
$$

e pare. $x^{j}$ e $\theta_{j}$ correspondem à extensão procurada.

5. Faça $\Delta^{\theta}=1$. Se $f\left(x^{j}+\Delta^{\theta}\left(v^{j}-v^{0}\right)\right) \geq f\left(x^{j}\right)$, a função $f(\cdot)$ cresce ilimitadamente na direção $v^{j}-v^{0}$; faça $\theta_{j}=\infty$ e pare. Do contrário, volte para o passo 4.

Esta possibilidade de aproveitamento de estrutura não é mencionada em [Thoai \& Tuy 1983].

De posse deste método para construção de $x^{j}$ e $\theta_{j}$, e fazendo uso de $f^{*}=0$ ao longo do algoritmo, é possível implementar os métodos de [Tuy 1964] e [Thoai \& Tuy 1983] aproveitando fortemente a estrutura do problema (LCP-PPC). $\mathrm{Na}$ seção seguinte, será visto como a estrutura do problema original (LCP) pode ser aproveitada ainda mais, a fim de definir cortes de concavidade mais profundos do que os da seção 3.1 . 


\subsection{Cortes Profundos para o LCP}

Nesta seção, o problema de programação côncava (LCP-PPC) é substituído por uma família de problemas de programação côncava (LCP- $\alpha \beta)$, onde $\alpha, \beta \in \mathbb{R}_{+}^{n}$ são escolhidos a cada iteração de modo a gerar cortes mais profundos do que os cortes de Tuy.

Definição 3.27 (LCP como PPC Paramétrico - LCP- $\alpha \beta$ )

$$
(L C P-\alpha \beta) \begin{cases}\min & f^{\alpha \beta}(z)=\sum_{i=1}^{n} \min \left\{\alpha_{i} z_{i}, \beta_{i} w_{i}(z)\right\} \\ & z \in X .\end{cases}
$$

Lema 3.28 (Equivalência entre (LCP) e (LCP- $\alpha \beta)$ )

Para quaisquer $\alpha, \beta \in \mathbb{R}_{++}^{n}$, o problema $(L C P-\alpha \beta)$ é equivalente ao (LCP), no sentido da proposição 3.22.

Prova: Seja $z \in X=\{z \geq 0 \mid w(z)=M z+q \geq 0\}$. Claramente $f^{\alpha \beta}(z)=\sum_{i=1}^{n} \min \left\{\alpha_{i} z_{i}, \beta_{i} w_{i}(z)\right\} \geq 0$. Além disso,

$$
f^{\alpha \beta}(z)=0 \Longleftrightarrow f(z)=0
$$

pois, como $z_{i}, w_{i}(z) \geq 0$ e $\alpha, \beta \in \mathbb{R}_{++}^{m}$,

$$
\min \left\{\alpha_{i} z_{i}, \beta_{i} w_{i}(z)\right\}=0 \Longleftrightarrow z_{i}=0 \text { ou } w_{i}(z)=0
$$

Deste modo, o (LCP- $\alpha \beta$ ) é equivalente ao problema (LCP-PPC), e conseqüentemente equivalente ao problema original (LCP), no sentido da proposição 3.22 .

Apesar da equivalência entre os problemas depender da hipótese forte $\alpha, \beta \in \mathbb{R}_{++}^{n}$, para efeito da construção de cortes de concavidade admite-se $\alpha, \beta \in \mathbb{R}_{+}^{n}$, 
desde que se tome o cuidado de garantir que $f^{\alpha \beta}\left(v^{0}\right)>0$ e assim, pelo lema 3.24 , garantir a corretude dos cortes produzidos.

No restante desta seção, $v^{0}$ é um mínimo local-* de $f(\cdot)$ em $X$ tal que $f\left(v^{0}\right)>0, v^{1}, v^{2}, \ldots, v^{n}$ são os vizinhos de $v^{0}$ e, para $\alpha, \beta \in \mathbb{R}_{+}^{n}$,

$$
\begin{aligned}
\theta_{j}^{\alpha \beta} & =\sup \left\{\theta \mid f^{\alpha \beta}\left(v^{0}+\theta\left(v^{j}-v^{0}\right)\right) \geq 0\right\} \\
\theta_{j} & =\sup \left\{\theta \mid f\left(v^{0}+\theta\left(v^{j}-v^{0}\right)\right) \geq 0\right\}, \\
I & =\left\{j \mid \theta_{j}<\infty\right\}, \mathrm{e} \\
x^{j} & = \begin{cases}v^{0}+\theta_{j}\left(v^{j}-v^{0}\right), & \text { se } j \in I, \\
v^{j} & \text { caso contrário. }\end{cases}
\end{aligned}
$$

A estratégia de aprofundamento de cortes aqui proposta consiste em, para cada $v^{0}, v^{1}, v^{2}, \ldots, v^{n}$ utilizados na definição do corte de concavidade, escolher os parâmetros $\alpha, \beta \in \mathbb{R}_{+}^{n}$ de modo que

$$
\theta_{j}^{\alpha \beta} \geq \theta_{j}, \forall j
$$

e tentar tornar esta desigualdade estrita para pelo menos um índice $j \in I$. A condição acima garante que o corte de Tuy obtido através da função $f^{\alpha \beta}(\cdot)$ é no mínimo tão profundo quanto o corte de Tuy obtido com a função $f(\cdot)$.

\section{Lema 3.29 (Caracterização de corte profundo I)}

Seja $a^{t} x \geq b$ o corte de concavidade associado a $v^{0}$ e $f(\cdot)$, como na definição 3.7, e seja $c^{t} x \geq d$ o corte de concavidade associado a $v^{0}$ e $f^{\alpha \beta}(\cdot)$, com $\alpha, \beta \in \mathbb{R}_{+}^{n}$. Se $\theta_{j}^{\alpha \beta} \geq \theta_{j}, \forall j$, então

$$
\forall x \in X \quad\left[c^{t} x \geq d \Longrightarrow a^{t} x \geq b\right]
$$

Prova : Considere inicialmente o caso $\theta_{j} \leq \theta_{j}^{\alpha \beta}<\infty$. Neste caso, $v^{0}+\theta_{j}\left(v^{j}-v^{0}\right) \in \operatorname{conv}\left\{v^{0}, v^{0}+\theta_{j}^{\alpha \beta}\left(v^{j}-v^{0}\right)\right\}$. Se, por outro lado, 


$$
\begin{aligned}
& \theta_{j}<\theta_{j}^{\alpha \beta}=\infty, \text { então } \theta_{j}\left(v^{j}-v^{0}\right) \in \operatorname{cone}\left\{v^{j}-v^{0}\right\} . \text { Portanto } \\
& \quad \operatorname{conv}\left\{v^{0}, v^{0}+\theta_{j}\left(v^{j}-v^{0}\right) \mid \theta_{j}<\infty\right\}+\operatorname{cone}\left\{v^{j}-v^{0} \mid \theta_{j}=\infty\right\} \\
& \quad \subseteq \operatorname{conv}\left\{v^{0}, v^{0}+\theta_{j}^{\alpha \beta}\left(v^{j}-v^{0}\right) \mid \theta_{j}^{\alpha \beta}<\infty\right\}+\operatorname{cone}\left\{v^{j}-v^{0} \mid \theta_{j}^{\alpha \beta}=\infty\right\}
\end{aligned}
$$

e, pelo lema 3.8

$$
\forall x \in X\left[c^{t} x \geq d \Longrightarrow a^{t} x \geq b\right]
$$

No intuito de tornar as desigualdades $\theta_{k}^{\alpha \beta} \geq \theta_{k}$ estritas, para $k \in I$, mantendo todas as outras desigualdades $\theta_{j}^{\alpha \beta} \geq \theta_{j}, \forall j$ válidas, é tentador escrever o problema de otimização

$$
\begin{cases}\max & \sum_{k \in I} \theta_{k}^{\alpha \beta} \\ \text { s.a. } & \theta_{j}^{\alpha \beta} \geq \theta_{j}, \forall j \\ & \alpha, \beta \geq 0 .\end{cases}
$$

É verificado a seguir que as restrições do problema acima podem ser formuladas como restrições lineares nas variáveis $\alpha$ e $\beta$.

Lema 3.30 (Caracterização de $\theta_{j}^{\alpha \beta} \geq \theta_{j} \neq \infty$ )

Suponha que $\theta_{j}<\infty$. Então são equivalentes

1. $\theta_{j}^{\alpha \beta} \geq \theta_{j}$

2. $f^{\alpha \beta}\left(x^{j}\right) \geq 0$;

3. $\sum_{i=1}^{n} \sigma_{i}^{j} \geq 0, \sigma_{i}^{j} \leq \alpha_{i} x_{i}^{j}, \sigma_{i}^{j} \leq \beta_{i} w_{i}\left(x^{j}\right), i=1,2, \ldots, n$.

Prova : $[1 \Longleftrightarrow 2]$ Definição de $\theta_{j}^{\alpha \beta}$ e concavidade de $f^{\alpha \beta}\left(x^{j}\right)$.

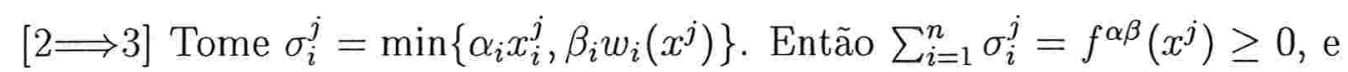
$\sigma_{i}^{j} \leq \alpha_{i} x_{i}^{j}, \sigma_{i}^{j} \leq \beta_{i} w_{i}\left(x^{j}\right), i=1,2, \ldots, n$.

$[3 \Longrightarrow 2] f^{\alpha \beta}\left(x^{j}\right)=\sum_{i=1}^{n} \min \left\{\alpha_{i} x_{i}^{j}, \beta_{i} w_{i}\left(x^{j}\right)\right\} \geq \sum_{i=1}^{n} \sigma_{i}^{j} \geq 0$. 
Lema 3.31 (Caracterização de $\theta_{j}^{\alpha \beta} \geq \theta_{j}=\infty$ )

Suponha que $\theta_{j}=\infty$. Então são equivalentes

1. $\theta_{j}^{\alpha \beta}=\infty$;

2. $f^{\alpha \beta}\left(v^{0}+\theta\left(v^{j}-v^{0}\right)\right) \geq 0, \forall \theta \geq 0$;

3. $\sum_{i=1}^{n} \min \left\{\alpha_{i}\left(v_{i}^{j}-v_{i}^{0}\right), \beta_{i} M_{i}\left(v^{j}-v^{0}\right)\right\} \geq 0$;

4. $\sum_{i=1}^{n} \sigma_{i}^{j} \geq 0, \sigma_{i}^{j} \leq \alpha_{i}\left(v_{i}^{j}-v_{i}^{0}\right), \sigma_{i}^{j} \leq \beta_{i} M_{i}\left(v^{j}-v^{0}\right), i=1,2, \ldots, n$.

Prova : Para abreviar a notação, considere $d=v^{j}-v^{0}$.

$[1 \Longleftrightarrow 2]$ Definição de $\theta_{j}^{\alpha \beta}$ e concavidade de $f^{\alpha \beta}\left(x^{j}\right)$.

[2 $\Longrightarrow 3]$ Seja $g(x)=\sum_{i=1}^{n} \min \left\{\alpha_{i} x_{i}, \beta_{i} M_{i} x\right\}$, e suponha por contradição que $g(d)<0$. Então $\lim _{\theta \rightarrow \infty} g(\theta d)=\lim _{\theta \rightarrow \infty} \theta g(d)=-\infty$. Como $g(\cdot)$ é côncava, pelo lema 3.9 tem-se $\lim _{\theta \rightarrow \infty} g\left(v^{0}+\theta d\right)=-\infty$. Mas

$$
\begin{aligned}
f^{\alpha \beta}\left(v^{0}+\theta d\right) & =\sum_{i=1}^{n} \min \left\{\alpha_{i}\left(v_{i}^{0}+\theta d_{i}\right), \beta_{i}\left(M_{i}\left(v^{0}+\theta d\right)+q_{i}\right)\right\} \\
& \left.\leq \sum_{i=1}^{n} \min \left\{\alpha_{i}\left(v_{i}^{0}+\theta d_{i}\right)+\beta_{i}\left|q_{i}\right|, \beta_{i}\left(M_{i}\left(v^{0}+\theta d\right)\right)+\beta_{i}\left|q_{i}\right|\right)\right\} \\
& =g\left(v^{0}+\theta d\right)+\sum_{i=1}^{n} \beta_{i}\left|q_{i}\right|,
\end{aligned}
$$

e $\operatorname{assim} \lim _{\theta \rightarrow \infty} f\left(v^{0}+\theta d\right) \leq \lim _{\theta \rightarrow \infty} g\left(v^{0}+\theta d\right)+\sum_{i=1}^{n} \beta_{i}\left|q_{i}\right|=-\infty$.

$[3 \Longrightarrow 2]$ Sejam

$$
\begin{aligned}
& I^{1}=\left\{i \mid \alpha_{i} d_{i}<\beta_{i} M_{i} d\right\} \mathrm{e} \\
& I^{2}=\left\{i \mid \alpha_{i} d_{i} \geq \beta_{i} M_{i} d\right\}
\end{aligned}
$$


Então

$$
\begin{aligned}
f^{\alpha \beta}\left(v^{0}+\theta d\right)= & \sum_{i=1}^{n} \min \left\{\alpha_{i}\left(v_{i}^{0}+\theta d_{i}\right), \beta_{i}\left(M_{i}\left(v^{0}+\theta d\right)+q_{i}\right)\right\} \\
\geq & \left.\sum_{i \in I} \min \left\{\alpha_{i} v_{i}^{0}+\alpha_{i} \theta d_{i}, \beta_{i}\left(M_{i} v^{0}+q_{i}\right)+\alpha_{i} \theta d_{i}\right)\right\} \\
& +\sum_{i \notin I} \min \left\{\alpha_{i} v_{i}^{0}+\beta_{i} \theta M_{i} d, \beta_{i}\left(M_{i} v^{0}+q_{i}\right)+\beta_{i} \theta M_{i} d\right\} \\
= & \sum_{i \in I} \alpha_{i} \theta d_{i}+\sum_{i \notin I} \beta_{i} \theta M_{i} d+\sum_{i=1}^{n} \min \left\{\alpha_{i} v_{i}^{0}, \beta_{i}\left(M_{i} v^{0}+q_{i}\right)\right\} \\
= & \theta \sum_{i=1}^{n} \min \left\{\alpha_{i} d_{i}, \beta_{i} M_{i} d\right\}+f\left(v^{0}\right) \\
\geq & f\left(v^{0}\right) \\
> & 0 .
\end{aligned}
$$

[3 $\Longleftrightarrow 4]$ Análoga à demonstração de [2 $\Longleftrightarrow 3]$ no lema 3.30.

O problema de otimização anteriormente proposto pode então ser reformulado como

$$
\left\{\begin{aligned}
\max \quad \sum_{k \in I} \theta_{k}^{\alpha \beta} & \\
\text { s.a. } \quad \sum_{i=1}^{n} \sigma_{i}^{j} & \geq 0 \\
\sigma_{i}^{j} & \leq \alpha_{i} x_{i}^{j} \quad j \in I, i=1,2, \ldots, n \\
\sigma_{i}^{j} & \leq \beta_{i} w_{i}\left(x^{j}\right), \\
\sum_{i=1}^{n} \sigma_{i}^{j} & \geq 0 \\
\sigma_{i}^{j} & \leq \alpha_{i}\left(v_{i}^{j}-v_{i}^{0}\right) \\
\sigma_{i}^{j} & \leq \beta_{i} M_{i}\left(v^{j}-v^{0}\right), \\
\alpha, \beta & \geq 0,
\end{aligned}\right.
$$

onde $\theta_{k}^{\alpha \beta}=\sup \left\{\theta \mid f^{\alpha \beta}\left(v^{0}+\theta\left(v^{k}-v^{0}\right)\right) \geq 0\right\}$ e $\theta_{k}<\infty$. Note que este problema é sempre viável, tomando-se $\alpha=\beta=\rrbracket \mathrm{e}$

$$
\sigma_{i}^{j}= \begin{cases}\min \left\{x_{i}^{j}, w_{i}\left(x^{j}\right)\right\} & j \in I \\ \min \left\{v_{i}^{j}-v_{i}^{0}, M_{i}\left(v^{j}-v^{0}\right)\right\} & j \notin I .\end{cases}
$$

A definição de $\theta_{k}^{\alpha \beta}$ é naturalmente modelada como $\max \theta$ sujeito a

$$
f^{\alpha \beta}\left(v^{0}+\theta_{k}\left(v^{k}-v^{0}\right)\right) \geq 0
$$


ou ainda,

$$
\left\{\begin{aligned}
\sum_{i=1}^{n} \sigma_{i}^{k} & \geq 0 \\
\sigma_{i}^{k} & \leq \alpha_{i} v_{i}^{0}+\alpha_{i} \theta\left(v_{i}^{k}-v_{i}^{0}\right) \\
\sigma_{i}^{k} & \leq \beta_{i}\left(M_{i} v^{0}+q_{i}\right)+\beta_{i} \theta\left(v_{i}^{k}-v_{i}^{0}\right) .
\end{aligned}\right.
$$

Nota-se que as restrições acima são não-lineares, e não-convexas para os índices $i$ tais que $v_{i}^{k}-v_{i}^{0}<0$. A dificuldade com a modelagem de $\theta_{k}$ sugere a substituição do objetivo $\max \sum_{k \in I} \theta_{k}$ por um objetivo mais modesto como, por exemplo, $\max \sum_{k \in I} f^{\alpha \beta}\left(x^{k}\right)$.

\section{Lema 3.32 (Caracterização de corte profundo II)}

Seja $a^{t} x \geq b$ o corte de concavidade associado a $v^{0}$ e $f(\cdot)$, como na definição 3.7, e seja $c^{t} x \geq d$ o corte de concavidade associado a $v^{0}$ e $f^{\alpha \beta}(\cdot)$, com $\alpha, \beta \in \mathbb{R}_{+}^{n}$. Se $f^{\alpha \beta}\left(x^{j}\right) \geq 0, \forall j: \theta_{j}<\infty, e f^{\alpha \beta}\left(v^{0}+\theta\left(v^{j}-v^{0}\right)\right) \geq 0, \forall \theta \geq 0, \forall j: \theta_{j}=\infty$, então

$$
\forall x \in X \quad\left[c^{t} x \geq d \Longrightarrow a^{t} x \geq b\right]
$$

Prova : As condições

$$
\begin{aligned}
f^{\alpha \beta}\left(x^{j}\right) & \geq 0, \quad \forall j: \theta_{j}<\infty, \mathrm{e} \\
f^{\alpha \beta}\left(v^{0}+\theta\left(v^{j}-v^{0}\right)\right) & \geq 0, \quad \forall \theta \geq 0, \forall j: \theta_{j}=\infty
\end{aligned}
$$

são equivalentes a $\theta_{j}^{\alpha \beta} \geq \theta_{j}, \forall j$, pelos lemas 3.30 e 3.31. Portanto, pelo lema 3.29 , tem-se o resultado.

Antes de apresentar o método para obtenção de cortes profundos para o (LCP), mostra-se que não há perda de generalidade em restringir a família de funções $f^{\alpha \beta}(\cdot)$ a vetores $\alpha, \beta$ em um compacto.

\section{Lema 3.33 (Restrição de $\alpha, \beta$ a um compacto)}

Seja $v^{0}$ um mínimo local-* de $f(\cdot)$ em $X$, e seja $a^{t} x \geq b$ o corte de Tuy associado a $f^{\alpha \beta}(\cdot)$ onde $\alpha, \beta \in \mathbb{R}_{+}^{n}$. Então existem $\alpha^{\prime}, \beta^{\prime} \in \mathbb{R}_{+}^{n}$ satisfazendo $\mathbb{1}^{t} \alpha^{\prime} \leq 1$ e $\mathbb{1}^{t} \beta^{\prime} \leq 1$, tais que $f^{\alpha^{\prime} \beta^{\prime}}(\cdot)$ produz o mesmo corte de Tuy $a^{t} x \geq b$. 
Prova : Suponha que $\mathbb{1}^{t} \alpha>1$, e seja

$$
\varrho=n \max \left\{\left|\alpha_{i}\right|,\left|\beta_{j}\right| \mid i, j=1,2, \ldots, n\right\}
$$

Defina $\alpha^{\prime}=(1 / \varrho) \alpha$ e $\beta^{\prime}=(1 / \varrho) \beta$; claramente $\mathbb{1}^{t} \alpha \leq 1$ e $\mathbb{1}^{t} \beta \leq 1$. Note que

$$
f^{\alpha^{\prime} \beta^{\prime}}(x)=\frac{1}{\varrho} f^{\alpha \beta}(x), \forall x \in \mathbb{R}^{n}
$$

Assim, como $f^{*}=0$, os valores $\theta_{j}^{\prime}, I^{\prime}$ e $x^{\prime j}$ calculados pela definição 3.7 para a função $f^{\alpha^{\prime} \beta^{\prime}}(\cdot)$ são idênticos aos valores $\theta_{j}, I$ e $x^{j}$ obtidos usando-se a função $f^{\alpha \beta}(\cdot)$ e, portanto, o corte de Tuy é o mesmo.

\section{Método para obtenção de corte profundo}

0. Seja $v^{0}$ um mínimo local-* de $f(\cdot)$ em $X$, com $f\left(v^{0}\right)=\bar{f}^{0}>0$. Faça $\alpha=\beta=1$.

1. Calcule

$$
\begin{aligned}
\theta_{j} & =\sup \left\{\theta \mid f^{\alpha \beta}\left(v^{0}+\theta\left(v^{j}-v^{0}\right)\right) \geq 0\right\}, \\
I & =\left\{j \mid \theta_{j}<\infty\right\}, \mathrm{e} \\
x^{j} & = \begin{cases}v^{0}+\theta_{j}\left(v^{j}-v^{0}\right), & \text { se } j \in I, \\
v^{j} & \text { caso contrário. }\end{cases}
\end{aligned}
$$


2. Resolva o PL nas variáveis $\alpha, \beta$ e $\sigma$,

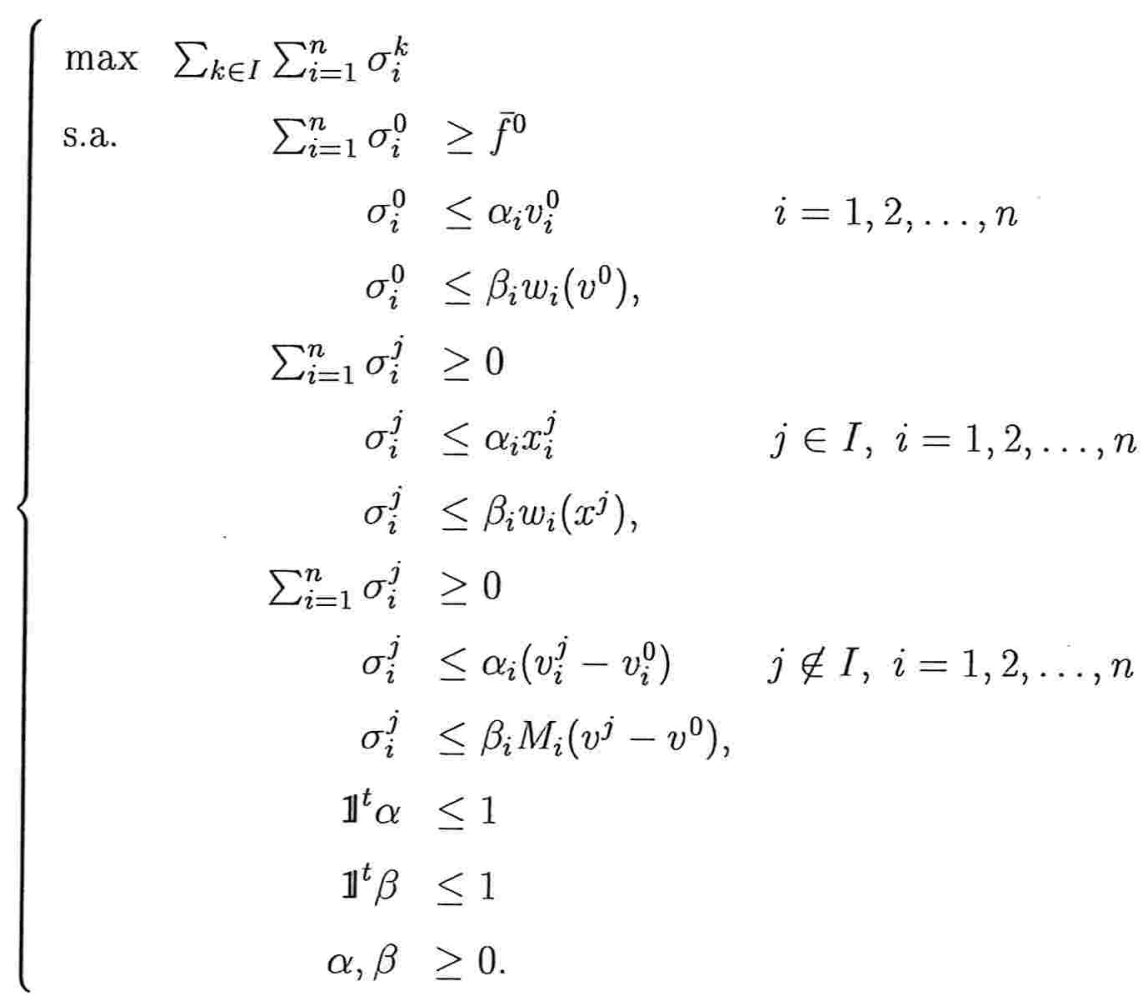

Seja $\left(\alpha^{*}, \beta^{*}, \sigma^{*}\right)$ a solução ótima do PL.

3. Faça $\alpha \leftarrow \alpha^{*}$ e $\beta \leftarrow \beta^{*}$. 
Proposição 3.34 (Corretude do método de obtenção de corte profundo) Seja $v^{0}$ um mínimo local- ${ }^{*}$ de $f(\cdot)$ em $X$ como na definição 3.7. Seja $a^{t} x \geq b$ o corte de concavidade associado a $v^{0}$ e $f(\cdot)$, e seja $c^{t} x \geq d$ o corte de concavidade obtido pelo método acima. Então, $\forall x \in X$,

$$
a^{t} x<b \Longrightarrow c^{t} x<d \Longrightarrow f^{\alpha \beta}(x)>0 \text {. }
$$

Além disso, ou o corte obtido pelo método é estritamente mais profundo do que o corte original, ou então o corte original é eficiente em relação à familia de funções $f^{\alpha \beta}(\cdot)$, no sentido que nenhuma função $f^{\alpha \beta}(\cdot)$ pode gerar um corte estritamente mais profundo do que $a^{t} x \geq b$.

Prova : O passo 2 do método gera um corte pelo menos tão profundo quanto o corte de Tuy, pelo lema 3.32 e pelas caracterizações dadas pelos lemas 3.30 e 3.31. Isso prova que $a^{t} x<b \Longrightarrow c^{t} x<d$, para todo $x \in X$. Além disso, $f^{\alpha \beta}\left(v^{0}\right) \geq \sum_{i=1}^{n} \sigma_{i}^{0} \geq f\left(v^{0}\right)>0$ e então, pelo lema 3.24 ,

$$
c^{t} x<d \Longrightarrow f^{\alpha \beta}(x)>0
$$

Suponha que o corte obtido pelo método não é estritamente mais profundo do que o corte original. Isso significa que na solução do PL no passo 3 do algoritmo, para todo $k \in I$, vale que $\max \sum_{i=1}^{n} \sigma_{i}^{k}=f\left(x^{k}\right)=0$, onde $x^{k}$ é a extensão original de Tuy $\operatorname{com} \alpha=\beta=1$, e assim $\theta_{k}^{\alpha^{*} \beta^{*}}=\theta_{k}$. Logo, as restrições $\theta_{j}^{\alpha \beta} \geq \theta_{j}, \forall j$, comuns a todos os PLs, forçam a satisfação de $\theta_{k}^{\alpha^{*} \beta^{*}}=\theta_{k}, \forall k \in I$, para todos os $\alpha, \beta \in \mathbb{R}_{+}^{n}$. Assim, pela contrapositiva do lema 3.29 , nenhuma função $f^{\alpha \beta}(\cdot)$ pode gerar um corte estritamente mais profundo do que o corte original de Tuy. 


\section{Observação 3.35 (Heurística para cortes profundos)}

Para LCPs em dimensão muito alta, o PL do passo 3 do método para obtenção de cortes profundos pode se tornar custoso computacionalmente, visto que o número de variáveis cresce quadraticamente com $n$. Nessa situação, é possível aplicar antes uma heurística de aprofundamento, detalhada a seguir.

Considere o método para obtenção de cortes profundos, seja $d^{j}=v^{j}-v^{0}$, e considere $f_{i}^{j}=\min \left\{\alpha_{i} x_{i}^{j}, \beta_{i} w_{i}\left(x^{j}\right)\right\}$ e $g_{i}^{j}=\min \left\{\alpha_{i} d^{j}, \beta_{i} M_{i} d^{j}\right\}$ no início do passo 3. Uma versão mais fraca daquele método pode ser obtida substituindo-se as condições

$$
\begin{aligned}
\sum_{i=1}^{n} \min \left\{\alpha_{i} x_{i}^{j}, \beta_{i} w_{i}\left(x^{j}\right)\right\} & \geq 0, \quad \forall j \in I \\
\sum_{i=1}^{n} \min \left\{\alpha_{i} d^{j}, \beta_{i} M_{i} d^{j}\right\} & \geq 0, \quad \forall j \notin I .
\end{aligned}
$$

pelas condições mais simples (e restritivas)

$$
\begin{aligned}
\min \left\{\alpha_{i} x_{i}^{j}, \beta_{i} w_{i}\left(x^{j}\right)\right\} & \geq f_{i}^{j}, \quad \forall j \in I, \forall i=1,2, \ldots, n \\
\min \left\{\alpha_{i} d^{j}, \beta_{i} M_{i} d^{j}\right\} & \geq d_{i}^{j}, \quad \forall j \notin I, \quad \forall i=1,2, \ldots, n .
\end{aligned}
$$

Estas últimas condições são equivalentes a

$$
\begin{aligned}
\alpha_{i} x_{i}^{j} & \geq f_{i}^{j}, \quad \forall j \in I, \forall i=1,2, \ldots, n \\
\beta_{i} w_{i}\left(x^{j}\right) & \geq f_{i}^{j}, \quad \forall j \in I, \quad \forall i=1,2, \ldots, n \\
\alpha_{i} d^{j} & \geq d_{i}^{j}, \quad \forall j \notin I, \forall i=1,2, \ldots, n \\
\beta_{i} M_{i} d^{j} & \geq d_{i}^{j}, \quad \forall j \notin I, \quad \forall i=1,2, \ldots, n .
\end{aligned}
$$

que são suficientes para garantir a validade das condições originais. Mas estas últimas condif̧oes, juntamente com $\alpha, \beta \geq 0$ e $\alpha, \beta \leq \mathbb{1}$ definem uma caixa de valores para $\alpha$ e $\beta$,

$$
B=\{(\alpha, \beta) \mid \underline{\alpha} \leq \alpha \leq \bar{\alpha} \text { e } \underline{\beta} \leq \beta \leq \bar{\beta}\}
$$


Assim, o problema $P L(k)$ pode ser substituído pelo problema linear separável

$$
\begin{cases}\max & \sum_{i=1}^{n} \sigma_{i}^{k} \\ \text { s.a. } & \sigma_{i}^{k} \leq \alpha_{i} x_{i}^{j} \\ & \sigma_{i} \leq \beta_{i} w_{i}\left(x^{j}\right), i=1,2, \ldots, n \\ & (\alpha, \beta) \in B\end{cases}
$$

que possui a solução ótima trivial

$$
\begin{aligned}
& \alpha_{i}= \begin{cases}\underline{\alpha_{i}} & \text { se } x_{i}^{j} \leq 0, \\
\overline{\alpha_{i}} & \text { caso contrário } ;\end{cases} \\
& \beta_{i}= \begin{cases}\underline{\beta_{i}} & \text { se } w_{i}\left(x^{j}\right) \leq 0, \\
\overline{\beta_{i}} & \text { caso contrário. }\end{cases}
\end{aligned}
$$

\section{Observação 3.36 (Pesos infinitos)}

O resultado do lema 3.33 afirma que não há perda de generalidade em considerarse os pesos $\alpha$ e $\beta$ dentro de um compacto, isto é, que não há nenhum corte de Tuy produzido por uma função $f^{\alpha \beta}(\cdot)$ com $\alpha, \beta \in \mathbb{R}_{+}^{n}$ que não seja produzido utilizandose os pesos dentro de um compacto. Isto não é o mesmo que afirmar que todos os cortes obtidos com $\alpha_{i}, \beta_{j} \in \mathbb{R} \cup\{\infty\}$ também podem ser produzidos com os pesos dentro de um compacto. O lema abaixo mostra que a adoção de pesos infinitos não acrescenta nenhuma generalidade ao que foi exposto anteriormente.

Com a adoção de pesos infinitos, a função $f^{\alpha \beta}(z)$ ainda é côncava, mas em geral perde-se a continuidade, pois

$$
\min \left\{\infty z_{i}, w_{i}(z)\right\}= \begin{cases}-\infty & \text { se } z_{i}<0 \\ \min \left\{0, w_{i}(z)\right\} & \text { se } z_{i}=0 \\ w_{i}(z) & \text { se } z_{i}>0\end{cases}
$$


Alguns cuidados se tornam necessários para impedir somas do tipo $\infty+(-\infty)$. Note-se ainda a necessidade da convenção $\infty 0=0$, para garantir a propriedade fundamental

$$
\forall z \in X, f^{\alpha \beta}(z)=0 \Longleftrightarrow f(z)=0
$$

Esta propriedade, juntamente com a concavidade $e$ o fato de que $\forall z \in X$, $f^{\alpha \beta}(z) \geq 0$, permitem a definição de um corte de concavidade com $\alpha_{i}=\infty$.

\section{Lema 3.37 (Pesos infinitos não acrescentam generalidade)}

Sejam $v^{0}, x^{1}, x^{2}, \ldots, x^{n}$ e $I=\left\{j \mid \theta_{j}<\infty\right\}$ como na definição 3. $7, \alpha, \beta \in(\mathbb{R} \cup\{\infty\})^{n}$ e seja $a^{t} x \geq b$ o corte produzido com a função $f^{\alpha \beta}(\cdot)$. Então existem $\alpha^{\prime}, \beta^{\prime} \in \mathbb{R}_{+}^{n}$ com $\mathbb{1}^{t} \alpha \leq 1$ e $\mathbb{1}^{t} \beta \leq 1$, tais que $f^{\alpha^{\prime} \beta^{\prime}}(\cdot)$ produz o mesmo corte.

Prova : Suponha que $\alpha_{k}=\infty$. A prova consiste em substituir este $\alpha_{k}$ por um número real $\alpha_{k}^{\prime}=\max \left\{u_{1}, u_{2}, \ldots, u_{n}\right\}$ definidos abaixo..

A definição de $x^{j}$ para $j \in I$ garante que

$$
f^{\alpha \beta}\left(x^{j}\right)=\sum_{i=1}^{n} \min \left\{\alpha_{i} x_{i}^{j}, \beta_{i} w_{i}\left(x^{j}\right)\right\}=0
$$

e, portanto, $x_{k}^{j} \geq 0$ e $\beta_{k} w_{k}\left(x^{j}\right)<\infty$. Para cada $j \in I$ defina

$$
u_{j}= \begin{cases}0 & \text { se } x_{k}^{j}=0 \\ \frac{\beta_{k} w_{k}\left(x^{j}\right)}{x_{k}^{j}} & \text { se } x_{k}^{j}>0\end{cases}
$$

Se $j \notin I$ então, sendo $d^{j}=v^{j}-v^{0}$,

$$
\sum_{i=1}^{n} \min \left\{\alpha_{i} d_{i}^{j}, \beta_{i} M_{i} d^{j}\right\} \geq 0
$$

e, portanto, $d_{k}^{j} \geq 0$. Para cada $j \notin I$ tal que $d_{k}^{j}=0$, defina $u_{j}=0$. Se $j \notin I$ é tal que $d_{k}^{j}>0$ e $\beta_{k} M_{k} d^{j}<\infty$, defina $u_{j}=\frac{\beta_{k} M_{k} d^{j}}{d_{k}^{j}}$. Se, por fim, 
$j \notin I, d_{k}^{j}>0$ e $\beta_{k} M_{k} d^{j}=\infty$, como a soma $\sum_{i=1}^{n} \min \left\{\alpha_{i} d_{i}^{j}, \beta_{i} M_{i} d^{j}\right\}$ não possui parcelas $-\infty$, existe um número real $u_{j}$ tal que

$$
\min \left\{u_{j} d_{k}^{j}, \beta_{k} M_{k} d^{j}\right\}+\sum_{i \neq k} \min \left\{\alpha_{i} d_{i}^{j}, \beta_{i} M_{i} d^{j}\right\} \geq 0
$$

Seja $\alpha_{i}^{\prime}=\alpha_{i}, \forall i \neq k$, e $\alpha_{k}^{\prime}=\max \left\{u_{1}, u_{2}, \ldots, u_{n}\right\}$. Por construção, $f^{\alpha^{\prime} \beta}\left(x^{j}\right)=f^{\alpha \beta}\left(x^{j}\right)=0$, para todo $j \in I$, e $\sum_{i=1}^{n} \min \left\{\alpha_{i}^{\prime} d_{i}^{j}, \beta_{i} M_{i} d^{j}\right\} \geq 0$, para todo $j \notin I$. Portanto $f^{\alpha^{\prime} \beta}(\cdot)$ gera o mesmo corte de Tuy que $f^{\alpha \beta}(\cdot)$. Repetindo o argumento para cada peso infinito, e aplicando ao final o lema 3.33 , tem-se o resultado.

\subsection{Experiência Computacional}

Os cortes profundos da seção anterior foram incorporados aos métodos de [Tuy 1964] e [Thoai \& Tuy 1983], e alguns testes aleatórios foram gerados para avaliar o impacto desta técnica. Os programas foram escritos em C, e executados em um Pentium III 1.2 $\mathrm{GHz}$ rodando Linux 2.2.19.

Os testes aleatórios foram gerados separadamente para LCPs com solução e LCPs sem solução. A garantia de solução pode ser obtida fazendo-se $q_{i} \geq-M_{i, n}, \forall i<n$ e $q_{n}=-M_{n, n}$, que fornece a solução $z=(0,0, \ldots, 0,1)^{t}$. Os problemas sem solução foram obtidos fazendo-se $w_{1}(z)=z_{1}-1$ e $w_{2}(z)=z_{1}-2$, o que garante que $z_{1} w_{1}>0$ em toda solução viável. A limitação do poliedro é garantida fazendo-se $w_{n}(z)=\sum_{i=1}^{n}\left(-\mu_{i}\right) z_{i}$, onde $\mu>0$. Foram descartados todos os testes resolvidos trivialmente, isto é, todos os testes para os quais uma solução foi obtida pelo método de Lemke, ou durante a inicialização por simplex.

No caso do método original de [Tuy 1964], foram adotados os seguintes procedimentos em situações de dificuldade. 
1. O método comporta até MAXCUTS $=20 n$ cortes, onde $n$ é a dimensão do (LCP).

2. Se o método de busca de mínimo local-* (página 3.1) termina em um vértice degenerado, este método é re-iniciado a partir de um novo vértice aleatório. Esta re-inicialização é permitida até um número máximo de MAXDEGEN=5 vezes por iteração externa.

3. Se, a partir de um mínimo local $v^{0}$, são obtidas as extensões $\left\{x^{i} \mid i=1,2, \ldots, n\right\}$, e o volume da região cortada é muito pequeno (menor que $10^{-8}$ ), então o corte mal-comportado não é introduzido, e um novo vértice aleatório é gerado para a busca de mínimo local, até um máximo de MAXBADCUTS=5 novos vértices por iteração externa.

É utilizado como estimativa do volume da região cortada o volume do simplexo com $n$ arestas ortogonais saindo de $v^{0}$ com comprimentos $\left\|x^{i}-v^{0}\right\|, i=1,2, \ldots, n$, dado por

$$
V\left(v^{0}, x^{1}, \ldots, x^{n}\right)=\frac{\prod_{i=1}^{n}\left\|x^{i}-v^{0}\right\|}{n !} .
$$

4. Se um dos limites acima é atingido, uma rotina de abandono de cortes é acionada, que ordena os valores $V\left(v^{0}, x^{1}, \ldots, x^{n}\right)$ dos cortes introduzidos, toma $\bar{V}$ o máximo dentre estes valores, e elimina todos os cortes cuja estimativa de volume seja menor que $\frac{\bar{V}}{10}$, retendo um número máximo de MAXCUTS/2 cortes. Esta rotina pode ser acionada até MAXRESTARTS=3 vezes, sendo que na tentativa seguinte a MAXRESTARTS o programa interrompe a execução.

O método de [Thoai \& Tuy 1983] não tem as mesmas dificuldades na presença de degenerescência ou cortes mal-comportados. Um limite superior de $\mathrm{MAXCONES}=20 n$ foi estabelecido para o número de subproblemas. 
Os resultados dos testes referentes a problemas com solução com o método de [Tuy 1964] são apresentados na tabela a seguir. Para cada dimensão testada, foram gerados 10 problemas com solução, para os quais nem o método de Lemke nem a primeira busca de mínimo local-* fornecem a solução do (LCP). Para cada tipo de corte utilizado (corte original de Tuy, heurística para cortes profundos e método para obtenção de corte profundo) são dados o número de desistências (problemas em que não se conseguiu achar solução após MAXRESTARTS reinicializações), o número médio de cortes utilizados, e o tempo médio. A dimensão máxima para o método de obtenção de cortes profundos foi escolhida em função da capacidade de processamento em memória (isto é, sem "swap") da implementação do simplex utilizada.

\begin{tabular}{|c|c|c|c|c|c|c|c|c|c|}
\hline \multirow{2}{*}{ 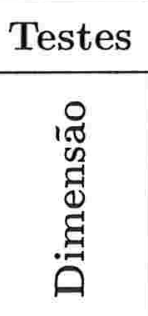 } & \multicolumn{3}{|c|}{ Cortes de Tuy } & \multicolumn{3}{|c|}{ Heur. p/ Cortes Prof. } & \multicolumn{3}{|c|}{ Cortes Profundos } \\
\hline & 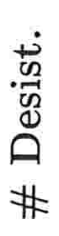 & 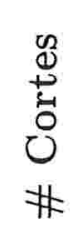 & 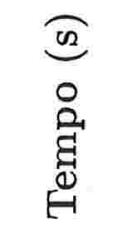 & 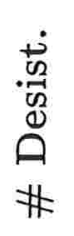 & 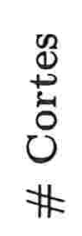 & 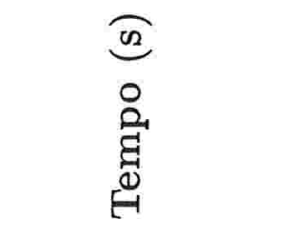 & 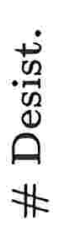 & 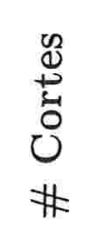 & 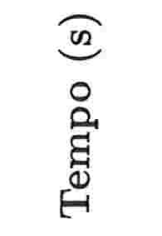 \\
\hline 5 & - & 1.3 & 0.002 & - & 1.0 & 0.002 & - & 1.2 & 0.009 \\
\hline 10 & - & 1.1 & 0.003 & - & 1.3 & 0.004 & - & 1.2 & 0.283 \\
\hline 15 & - & 3.9 & 0.043 & - & 1.3 & 0.019 & - & 2.2 & 8.443 \\
\hline 20 & - & 1.0 & 0.061 & - & 4.0 & 0.080 & 1 & 2.33 & 90.378 \\
\hline 30 & - & 1.5 & 1.317 & - & 1.7 & 0.448 & - & - & - \\
\hline 40 & - & 2.7 & 3.073 & - & 3.3 & 2.540 & - & - & - \\
\hline 50 & - & 1.1 & 8.372 & - & 6.2 & 9.244 & - & - & - \\
\hline
\end{tabular}

Os resultados do mesmo método para problemas sem solução são dados a seguir. 
Método de [Tuy 1964] p/ Problemas Sem Solução

\begin{tabular}{|c|c|c|c|c|c|c|c|c|c|}
\hline \multirow{2}{*}{ 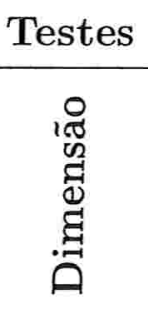 } & \multicolumn{3}{|c|}{ Cortes de Tuy } & \multicolumn{3}{|c|}{ Heur. p/ Cortes Prof. } & \multicolumn{3}{|c|}{ Cortes Profundos } \\
\hline & 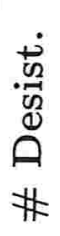 & 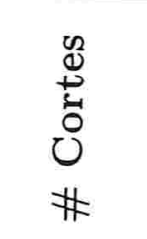 & 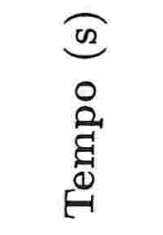 & 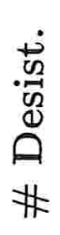 & 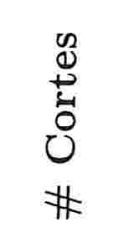 & $\begin{array}{l}\text { (2) } \\
\circ \\
\stackrel{0}{g} \\
\stackrel{0}{0}\end{array}$ & $\begin{array}{l}\dot{\vec{n}} \\
\stackrel{n}{0} \\
\stackrel{0}{0} \\
\text { @ } \\
\#\end{array}$ & 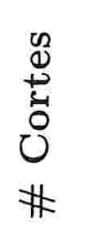 & 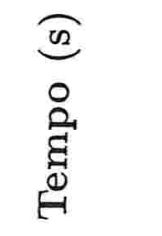 \\
\hline 4 & - & 1.3 & 0.001 & - & 1.0 & 0.001 & & 1.0 & 0.002 \\
\hline 6 & - & 6.6 & 0.003 & - & 1.7 & 0.001 & - & 1.0 & 0.008 \\
\hline 8 & 3 & 150.71 & 25.938 & - & 5.9 & 0.004 & - & 1.4 & 0.056 \\
\hline 10 & 8 & 259.50 & 236.802 & 3 & 234.14 & 84.017 & - & 1.3 & 0.132 \\
\hline
\end{tabular}

Nota-se, a partir das duas tabelas, que o aprofundamento dos cortes tem um impacto bastante diverso em problemas com e sem solução. Na última tabela (problemas sem solução), o impacto do aprofundamento é intuitivo, no sentido que o número de cortes necessários para esvaziar o poliedro diminui em função da "qualidade" do corte (o corte "profundo" é melhor do que o corte "heurístico", que por sua vez é melhor do que o corte de Tuy). Na primeira tabela, o impacto é menos previsível, pois uma mínima alteração em um corte pode conduzir o algoritmo diretamente à solução (obtida pela busca de mínimo local-*), ou afastá-lo completamente da solução.

Com exceção dos testes com solução em dimensões 30, 40 e 50, o desempenho deste método com qualquer escolha de corte é pior do que o desempenho de um método de solução direta, do tipo "branch \& bound". Lembra-se ainda que o método de [Tuy 1964] não possui qualquer resultado de convergência em geral.

A seguir, são apresentados os resultados da experiência computacional com o método de [Thoai \& Tuy 1983], para problemas com solução. As informações dadas são semelhantes às dadas para o método de [Tuy 1964]. Aqui o número de cortes calculados é igual ao número de cones enumerados no processo de bissecção. 
Método de [Thoai \& Tuy 1983] p/ Problemas Com Solução

\begin{tabular}{|c|c|c|c|c|c|c|c|c|c|}
\hline \multirow{2}{*}{ 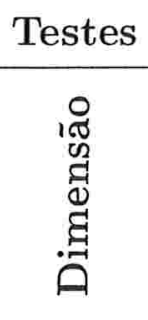 } & \multicolumn{3}{|c|}{ Cortes de Tuy } & \multicolumn{3}{|c|}{ Heur. p/ Cortes Prof. } & \multicolumn{3}{|c|}{ Cortes Profundos } \\
\hline & $\begin{array}{l}\dot{0} \\
\stackrel{0}{0} \\
\dot{D} \\
\stackrel{0}{A} \\
\#\end{array}$ & $\begin{array}{l}\text { on } \\
\stackrel{0}{\sharp} \\
0 \\
U \\
\#\end{array}$ & 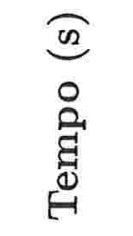 & 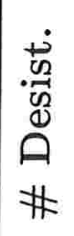 & 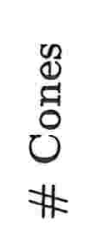 & 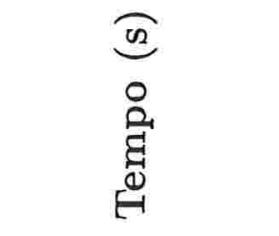 & 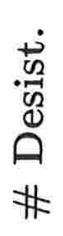 & $\begin{array}{l}\text { Oे } \\
\text { ఏ } \\
0 \\
\text { U } \\
\#\end{array}$ & 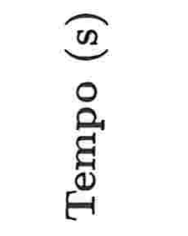 \\
\hline 5 & - & 1.6 & 0.002 & - & 1.0 & 0.002 & - & 1.6 & 0.012 \\
\hline 10 & - & 22.7 & 0.103 & - & 6.5 & 0.029 & - & 1.8 & 0.427 \\
\hline 15 & 1 & 2.78 & 1.736 & 1 & 2.11 & 1.888 & - & 3.1 & 5.903 \\
\hline 20 & - & 5.5 & 0.335 & - & 2.4 & 0.101 & - & 2.2 & 20.225 \\
\hline 30 & - & 3.6 & 0.636 & - & 4.6 & 1.135 & - & - & - \\
\hline 40 & - & 3.1 & 2.185 & - & 3.8 & 2.862 & - & - & - \\
\hline 50 & - & 3.6 & 7.267 & - & 4.6 & 9.777 & - & - & - \\
\hline
\end{tabular}

O mesmo método para problemas sem solução teve o comportamento descrito pela tabela a seguir.

Método de [Thoai \& Tuy 1983] p/ Problemas Sem Solução

\begin{tabular}{|c|c|c|c|c|c|c|c|c|c|}
\hline Testes & \multicolumn{3}{|c|}{ Cortes de Tuy } & \multicolumn{3}{|c|}{ Heur. p/ Cortes Prof. } & \multicolumn{3}{|c|}{ Cortes Profundos } \\
\hline 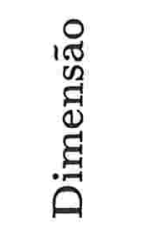 & 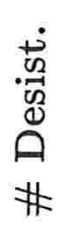 & $\begin{array}{l}\text { y } \\
0 \\
0 \\
0 \\
0 \\
\#\end{array}$ & 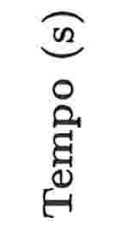 & 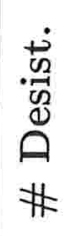 & 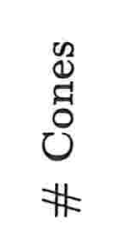 & 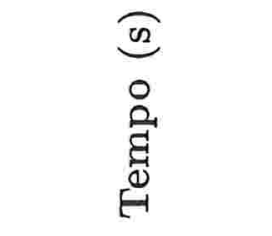 & 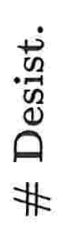 & 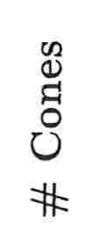 & 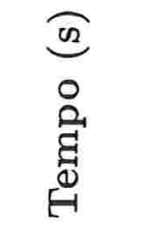 \\
\hline 4 & - & 6.4 & 0.001 & - & 1.0 & 0.001 & - & 1.0 & 0.002 \\
\hline 6 & 7 & 72.33 & 0.121 & 1 & 15.89 & 0.026 & - & 1.0 & 0.007 \\
\hline 8 & 10 & - & - & 8 & 151 & 0.526 & - & 30.2 & 1.518 \\
\hline 10 & 10 & - & - & 10 & - & - & 1 & 1.0 & 6.237 \\
\hline
\end{tabular}


As observações principais a partir dos testes acima são semelhantes às feitas para o método de [Tuy 1964]. No caso de problemas sem solução, observa-se aqui uma mudança qualitativa através dos cortes profundos, pois um grande número de problemas só puderam ser resolvidos através do uso destes cortes.

No entanto vale relembrar que, para as dimensões aqui consideradas, métodos diretos do tipo branch \& bound [Al-Khayyal 1987] são eficientes para a solução do (LCP). Resultados computacionais encorajadores para LCPs de dimensão $n$ entre 10 e 500 foram obtidos em [Pardalos \& Rosen 1988]. Isto parece indicar que existam alternativas mais interessantes para a solução de LCPs difíceis (em especial, quando a matriz é Não- $S_{0}$ ) do que aquela proposta em [Thoai \& Tuy 1983].

\subsection{Conclusão e Trabalho Futuro}

Neste capítulo foi apresentada uma técnica de aprofundamento de cortes de concavidade, inspirada pelos trabalhos de [Tuy 1964, Thoai \& Tuy 1980, Thoai \& Tuy 1983]. Constata-se que esta técnica produz cortes mais profundos do que os de Tuy, sempre que isto é possível. No entanto, a experiência computacional com os métodos originais, bem como com o uso de cortes aprofundados, não permite concluir que estes métodos sejam adequados para resolver LCPs de médio e grande porte.

O método original de [Tuy 1964] não possui resultados de convergência, embora possa ser considerado como um método heurístico para obtenção de mínimos locais-* de um problema de programação côncava (seção 3.1). No caso do (LCP), a obtenção de um mínimo local-* $z$ tal que $f(z)>0$ não dá informação nenhuma sobre o problema.

O método de [Thoai \& Tuy 1983], por outro lado, possui um resultado de convergência (proposição 3.19). No entanto, esta convergência pode ser muito lenta, como indicado no final da seção 3.1. Além disso, em comparação com o método branch 
\& bound para o (LCP), tem-se que este último impõe um dado forte sobre a solução pretendida, exigindo que $z_{i}=0$ ou $w_{i}=0$, e diminui estritamente a dimensão do subproblema, enquanto o processo de bissecção de cones de [Thoai \& Tuy 1983] divide o problema em dois, porém não diminui a dimensão do subproblema, nem ganha qualquer outra informação sobre as soluções, além daquela dada pela concavidade da função $f(\cdot)$. Isso pode explicar que também este método tenha interesse para problemas de programação côncava em geral, mas possa ser de pouco interesse para a solução do (LCP). 


\section{Capítulo 4}

\section{Conclusão Geral}

Neste trabalho foram considerados três problemas, a saber, o Problema de Ajuste de Dobradiças (capítulo 1), o Problema de Valores Próprios Complementar (capítulo 2) e o Problema de Complementaridade Linear (capítulo 3). Como as possíveis extensões de pesquisa já foram apresentadas no final de cada capítulo, são indicadas a seguir as principais contribuições do presente trabalho.

O Problema de Ajuste de Dobradiças surgiu em [Breiman 1993], onde foi proposto um método iterativo para encontrar mínimos locais do problema. Em [Pucar \& Sjöberg 1998] propôs-se uma técnica de amortização do tamanho de passo para aumentar o domínio de convergência do método. A formulação do problema utilizada nestas duas referências é

$$
\min _{x, y} \sum_{i=1}^{n}\left(\max \left\{A_{i} x, A_{i} y\right\}-b_{i}\right)^{2}
$$


A formulação do problema original como MPEC,

$$
(\mathrm{HF}) \begin{cases}\min & \sigma^{t} \sigma \\ \text { s.a. } & r=\sigma-A x+b \\ & s=\sigma-A y+b \\ & r^{t} s=0 \\ & r, s \geq 0\end{cases}
$$

é apresentada aqui pela primeira vez. Todas as caracterizações de soluções e propriedades desta formulação são novos. Também são novos o método enumerativo usando partições separáveis, e o conseqüente resultado de polinomialidade do número de nós da árvore de enumeração, para dimensão $m$ do espaço dos pontos dados fixa.

A formulação do problema com a norma $l_{1}$ como MPEC linear,

$$
(\mathrm{LHF}) \begin{cases}\min & \mathbb{1}^{t} \gamma \\ \text { s.a. } & r=\sigma-A x+b \\ & s=\sigma-A y+b \\ & \gamma \geq \sigma \\ & \gamma \geq-\sigma \\ & r^{t} s=0 \\ & r, s \geq 0,\end{cases}
$$

bem como a técnica de solução baseada em SLCP [Júdice \& Faustino 1992], aparecem aqui pela primeira vez.

O Problema de Valores Próprios Complementar foi proposto no "17th International Symposium on Mathematical Programming" [Júdice, Costa, Figueiredo \& Martins 2000]. Neste simpósio foram apresentados alguns resultados ${ }^{1}$ em colaboração com M. Queiroz e C. Humes. Outras proposições ${ }^{2}$ aparecem aqui pela primeira vez

\footnotetext{
${ }^{1}$ Proposições 2.12, 2.13, 2.15, 2.16, 2.17, 2.29 e 2.30.

${ }^{2}$ Proposições $2.14,2.18,2.21,2.22,2.23,2.24,2.25,2.26$ e 2.31
} 
e, em particular, o estudo do caso simétrico, que conduziu à solução via métodos de gradiente.

A proposta do uso de cortes de concavidade para a formulação do (LCP) como

$$
(L C P-P P C) \begin{cases}\min & f(z)=\sum_{i=1}^{n} \min \left\{z_{i}, w_{i}(z)\right\} \\ & z \in X\end{cases}
$$

é de [Thoai \& Tuy 1983]. A maioria dos resultados das seções 3.1 e 3.2 são diretamente derivados de [Tuy 1964, Thoai \& Tuy 1980, Thoai \& Tuy 1983]; as exceções são comentadas a seguir.

A limitação

$$
d_{k_{j+\frac{n(n-1)}{2}}} \leq \frac{d_{k_{j}}}{2}
$$

obtida no lema 3.17, bem como o uso da estrutura linear por trechos de $f^{j}(\theta)=\left(v^{0}+\theta\left(v^{j}-v^{0}\right)\right)$ (lema 3.26 e "método de construção de $x^{j}$ e $\theta_{j}$ para o (LCPPPC)"), não são mencionados em [Thoai \& Tuy 1983]. Além disso, a caracterização da classe de matrizes para as quais $X=\{z \geq 0 \mid w(z)=M z+q \geq 0\} \neq \emptyset$ é limitado (lema 3.23) não é mencionada nos trabalhos seguintes, que tratam a compacidade de $X$ como uma hipótese sobre $(M, q)$ : [Mangasarian 1997, Pardalos \& Rosen 1988, Sherali, Krishnamurty \& Al-Khayyal 1996, Sherali, Krishnamurty \& Al-Khayyal 1998, Thoai \& Tuy 1983].

A formulação do (LCP) como uma família de problemas de programação côncava

$$
(L C P-\alpha \beta) \begin{cases}\min & f^{\alpha \beta}(z)=\sum_{i=1}^{n} \min \left\{\alpha_{i} z_{i}, \beta_{i} w_{i}(z)\right\} \\ & z \in X,\end{cases}
$$

bem como a proposta de cortes profundos para o (LCP) baseados na escolha de membros desta família, aparecem aqui pela primeira vez. Outros resultados ${ }^{3}$, bem como os métodos de obtenção de cortes profundos, são novos.

\footnotetext{
${ }^{3}$ Lemas 3.28, 3.29, 3.30, 3.31, 3.32, 3.33, 3.37 e proposição 3.34
} 
É interessante observar a variedade de situações em complementaridade e de técnicas em programação matemática encontradas neste estudo. Para o MPEC do capítulo 1, após várias tentativas com outras abordagens, a melhor solução encontrada (e aqui apresentada) é um método de otimização combinatória (do tipo branch \& bound) explorando a relação entre soluções viáveis e hiperplanos separadores dos dados (partições separáveis). No capítulo 2, estabelece-se a equivalência entre um caso especial de um NCP misto e um problema de encontrar mínimos locais de um PNL. No capítulo 3, o LCP é tratado como um problema de otimização global (programação côncava). Nos três problemas, são encontradas formulações de subproblemas como PLs e PQs. Assim, considera-se que o presente trabalho cobre um amplo espectro do ferramental das áreas de complementaridade e programação matemática. 


\section{Referências Bibliográficas}

[Al-Khayyal 1987]

[Alon \& Onn 1999]

[Andreani \& Martinez 2001]

[Auchmuty 1989]

[Auchmuty 1991]

\begin{abstract}
AL-KHAYYAL, F. A.; An Implicit Enumeration Procedure for the General Linear Complementarity Problem. Mathematical Programming Study, 31:1-20, 1987.

ALON, N.; ONN, S.; Separable Partitions. Discrete Applied Mathematics, 91(1):39-51, 1999.
\end{abstract}

ANDREANI, R.; MARTINEZ, J. M.; On the Solution of Mathematical Programming Problems with Equilibrium Constraints by Means of Nonlinear Programming Algorithms. To appear in Mathematical Methods of Operations Research.

AUCHMUTY, G.; Unconstrained Variational Principles for Eigenvalues of Real Symmetric Matrices. SIAM Journal on Mathematical Analysis, 20(5):1186-1207, 1989.

AUCHMUTY, G.; Globally and Rapidly Convergent Algorithms for Symmetric Eigenpro- 
blems. SIAM Journal on Matrix Analysis and Applications, 12(4):690-706, 1991.

[Bai \& Evans 1997]

[Bard 1996]

[Bertsekas \& Tsitsiklis 1996]

[Breiman 1993]

[Chatelin 1993]

[Cichocki \& Unbehauen 1996]

[Costa, Figueiredo, Júdice \& Martins 2001]
BAI, Z.-Z.; EVANS, D. J.; Matrix Multisplitting Relaxation Methods for Linear Complementarity Problems. International Journal of Computer Mathematics, 63:309-326, 1997.

BARD, J.; Practical Bilevel Optimization: Algorithms and Applications. Kluwer Academic Publishers, 1998.

BERTSEKAS, D. P.; TSITSIKLIS, J. N.; Neuro-Dynamic Programming. Athena Scientific, Belmont, Massachusetts, 1996.

BREIMAN, L.; Hinging Hyperplanes for Regression, Classification and Function Approximation. IEEE Transactions on Information Theory, 39(3):999-1013, 1993.

CHATELIN, F.; Eigenvalues of Matrices. John Wiley \& Sons, 1993.

CICHOCKI, A.; UNBEHAUEN, R.; Neural Networks for Optimization and Signal Processing. John Wiley \& Sons, 1996.

] COSTA, A. P.; FIGUEIREDO, I. N.; JÚDICE, J.; MARTINS, J. A. C.; A Complementary Eigenproblem in the Stability Analysis of FiniteDimensional Elastic Systems with Frictional 
[Cottle 1976]

COTTLE, R. W.; Complementarity and Variational Problems. Symposia Mathematica, 19:177-208, 1976.

[Cottle \& Dantzig 1968]

COTTLE, R. W.; DANTZIG, G. B.; Complementarity Pivot Theory of Mathematical Programming. Linear Algebra and Its Applications, 1:103-125, 1968.

[Cottle \& Dantzig 1970]

COTTLE, R. W.; DANTZIG, G. B.; A Generalization of the Linear Complementarity Problem. Journal of Combinatorial Theory, 8:7990, 1970.

[Cottle \& Habetler 1970] COTTLE. R. W.; HABETLER, G. J.; On Classes of Copositive Matrices. Linear Algebra and its Applications, 3:295-310, 1970.

[Cottle, Pang \& Stone 1992]

COTTLE, R. W.; PANG, J. S.; STONE, R. E.; The Linear Complementarity Problem. Academic Press, 1992.

[Cover 1965] COVER, T. M.; Geometrical and Statistical Properties of Systems of Linear Inequalities with Applications in Patter Recognition. IEEE Transactions on Electronic Computers, 14:326$334,1965$. 
[Dirkse \& Ferris 1995]

[Eaves 1971]

[Floudas 2000]

[Floudas, Pardalos et alii. 1999]

[Fukushima, Luo \& Pang 1998]

[Ha 1985]
DIRKSE, S. P.; FERRIS, M. C.; The PATH Solver: A Nonmonotone Stabilization Scheme for Mixed Complementarity Problems. Optimization Methods and Software, 5:123-156, 1995.

EAVES, B. C.; The Linear Complementarity Problem. Management Science, 17(9):612-634, 1971.

FLOUDAS, C. A.; Deterministic Global Optimization: Theory, Methods and Applications. Kluwer Academic Publishers, 2000.

FLOUDAS, C. A.; PARDALOS, P. M.; ADJIMAN, C. S.; ESPOSITO, W. R.; GÜMÜS, Z. H.; HARDING, S. T.; KLEPEIS, J. L.; MEYER, C. A.; SCHWEIGER, C. A.; Handbook of Test Problems in Local and Global Optimization. Kluwer Academic Publishers, 1999.

FUKUSHIMA, M.; LUO, Z.-Q.; PANG, J.S.; A Globally Convergent Sequential Quadratic Programming Algorithm for Mathematical Programs with Linear Complementarity Constraints. Computational Optimization and Applications, 10:5-34, 1998.

HA, C. D.; Stability of the Linear Complementarity Problem at a Solution Point. Mathematical Programming, 31:327-338, 1985. 
[Harding 1967]

[Harker \& Pang 1990]

[Hassoun 1995]

[Horst, Pardalos \& Thoai 1995]

[Horst \& Tuy 1993]

[Júdice 1994]
HARDING, E.F.; The Number of Partitions of a Set of $\mathrm{n}$ Points in $\mathrm{k}$ Dimensions Induced by Hyperplanes. Proceedings of the Edinburgh Mathematical Society, 15(2):285-289, 1967.

HARKER, P. T.; PANG, J.-S.; FiniteDimensional Variational Inequality and Nonlinear Complementarity Problems: A Survey of Theory, Algorithms and Applications. Mathematical Programming - Series B - Variational Inequality Problems, 48(2):161-220, 1990.

HASSOUN, M. H.; Fundamentals of Artifical Neural Networks. MIT Press, Cambridge, Massachusetts, 1995.

HORST, R.; PARDALOS, P. M.; THOAI, N. V; Introduction to Global Optimization. Kluwer Academic Press, 1995.

HORST, R.; TUY, H.; Global Optimization: Deterministic Approaches. Springer Verlag, 1993.

JÚDICE, J.; Algorithms for Linear Complementarity Problems. In.: Algorithms for Continuous Optimization, Editor: E. Spedicato, 435-474. Kluwer Academic Publishers, 1994.

[Júdice, Costa, Figueiredo \& Martins 2000] JÚDICE, J.; COSTA, A. P.; FIGUEIREDO, I. N.; MARTINS, J. A. C.; The Eigenvalue Complementarity Problem: Algorithms and 
Application in Elastic Systems with Frictional Contact. In: 17th International Symposium on Mathematical Programming, Atlanta, August 7-11 2000. Mathematical Programming Society.

[Júdice \& Faustino 1988a]

JÚDICE, J.; FAUSTINO, A. M.; The Solution of the Bilevel Programming Problem by Using the Linear Complementarity Problem. Investigação Operacional, 8(1):77-95, 1988.

[Júdice \& Faustino 1988b]

JÚDICE, J.; FAUSTINO, A. M.; An Experimental Investigation of Enumerative Methods for the Linear Complementarity Problem. Computers and Operations Research, 15(5):417-426, 1988.

[Júdice \& Faustino 1991]

JÚDICE, J.; FAUSTINO, A. M.; A Computational Analysis of LCP Methods for Bilinear and Concave Quadratic Programming. Computers and Operations Research, 18(8):645654, 1991.

[Júdice \& Faustino 1992]

JÚDICE, J.; FAUSTINO, A. M.; A Sequential LCP Method for Bilevel Linear Programming. Annals of Operations Research, 34:89106, 1992.

[Júdice, Machado \& Faustino 1993] JÚDICE, J.; MACHADO, J.; FAUSTINO, A. M.; Extensão do Método de Lemke Para a Resolução do Problema Linear Complementar 
[Júdice \& Mitra 1988]

[Júdice \& Pires 1992]

[Júdice \& Pires 1994]

[Karamardian 1969]

[Karamardian 1971] com Limites Superiores. Portugaliae Mathematica, 50(2):205-216, 1993.

JÚDICE, J.; MITRA, G.; Reformulation of Mathematical Programming Problems as Linear Complementarity Problems and Investigation of Their Solution Methods. Journal of Optimization Theory and Applications, 57(1):123$149,1988$.

JÚDICE, J.; PIRES, F. M.; Basic-Set Algorithm for a Generalized Linear Complementarity Problem. Journal of Optimization Theory and Applications, 74(3):391-411, 1992.

JÚDICE, J.; PIRES, F. M.; A Block Principal Pivoting Algorithm for Large-Scale Strictly Monotone Linear Complementarity Problems. Computers and Operations Research, 21(5):587-596, 1994.

KARAMARDIAN, S.; The Nonlinear Complementarity Problem with Applications, part I and II. Journal of Optimization Theory and Applications, 4:87-98,167-181, 1969.

KARAMARDIAN, S.; Generalized Complementarity Problem. Journal of Optimization Theory and Applications, 8:161-167, 1971. 
[Karamardian 1972]

[Konno, Thach \& Tuy 1997]

[Lemarechal \& Urruty 1970]

[Luo, Pang \& Ralph 1996]

[Luo, Pang, Ralph \& Wu 1996]

[Mangasarian 1997]

[Mangasarian \& Pang 1997]
KARAMARDIAN, S.; The Complementarity Problem. Mathematical Programming, 2:107129, 1972.

KONNO, H.; THACH, P. T.; TUY, H.; Optimization on Low Rank Nonconvex Structures. Kluwer Academic Publishers, 1997.

LEMARECHAL; URRUTY; Convex Analysis and Minimization Algorithms, 2 vols. Springer Verlag, 1970.

LUO, Z.-Q.; PANG, J.-S.; RALPH, D.; Mathematical Programs with Equilibrium Constraints. Cambridge University Press, 1996.

LUO, Z.-Q.; PANG, J.-S.; RALPH, D.; WU, S.-Q.; Exact Penalization and Stationary Conditions of Mathematical Programs with Equilibrium Constraints. Mathematical Programming, 75(1):19-76, 1996.

MANGASARIAN, O. L.; Solution of General Linear Complementarity Problems via Nondifferentiable Concave Minimization. Acta Mathematica Vietamica, 22(1):199-205, 1997.

MANGASARIAN, O. L.; PANG, J. S.; Exact Penalty Functions for Mathematical Programs with Linear Complementarity Constraints. $O p$ timization, 42(1):1-8, 1997. 
[Mongeau \& Torki 1999]

[Moré 1974]

[Murtagh \& Saunders 1978]

[Murtagh \& Saunders 1987]

[Murty 1988]

[Ortega 1987]

[Ortega \& Rheinboldt 1970]
MONGEAU, M.; TORKI, M.; Computing Eigenelements of Real Symmetric Matrices via Optimization. Technical Report MIP 99-54, Université Paul Sabatier, Toulouse, 1999.

MORÉ, J. J.; Classes of Functions and Feasibility Conditions in Nonlinear Complementarity Problems. Mathematical Programming, 6:327338,1974 .

MURTAGH, B. A.; SAUNDERS, M. A.; Large-Scale Linearly Constrained Optimization, Mathematical Programming, 14:41-72, 1978.

MURTAGH, B. A.; SAUNDERS, M. A.; MINOS 5.1 User's Guide, Report SOL 83-20R, Department of Operations Research, Stanford University, 1987.

MURTY, K. G.; Linear Complementarity, Linear and Nonlinear Programming. Heldermann Verlag, Berlin, 1988.

ORTEGA, J. M.; Matrix Analysis: A Second Course. Plenum Press, New York, 1987.

ORTEGA, J. M.; RHEINBOLDT, W. C.; Iterative Solutions of Nonlinear Equations in Several Variables. Academic Press, New York, 1970. 
[Pardalos \& Rosen 1987]

[Pardalos \& Rosen 1988]

[Parlett 1997]

[Peng 1995]

[Pucar \& Sjöberg 1998]

[Ralph 1994]

[Ramarao \& Shetty 1984]
PARDALOS, P. M.; ROSEN, J. B.; Constrained Global Optimization: Algorithms and Applications. Lecture Notes in Computer Science, 268. Springer-Verlag Berlin, 1987.

PARDALOS, P. M.; ROSEN, J. B.; Global Optimization Approach to the Linear Complementarity Problem. SIAM Journal on Scientific and Statistical Computing, 9(2):341-353, 1988.

PARLETT, B. N.; The Symmetric Eigenvalue Problem. Classics in Applied Mathematics 20, SIAM, 1997.

PENG, J. M.; Unconstrained Optimization Methods for Nonlinear Complementarity Problem. Journal on Computational Mathematics, 13(3):259-266, 1995.

PUCAR, P.; SJÖBERG, J.; On the Hinge Finding Algorithm for Hinging Hyperplanes. IEEE Transactions on Information Theory, 44(3):1310-1318, 1998.

RALPH, D.; Global Convergence of Damped Newton's Method for Nonsmooth Equations via the Path Search. Mathematics of Operations Research, 19(2):352-389, 1994.

RAMARAO, B.; SHETTY, C. M.; Application of Disjunctive Programming to the Line- 
[Robinson 1979]

[Robinson 1980]

ROBINSON, S. M.; Strongly Regular Generalized Equations. Mathematics of Operations Research, 5(1):43-62, 1980.

[Robinson 1981]

ROBINSON, S. M.; Some Continuity Properties of Polyhedral Multifunctions. Mathematical Programming Study, 14:206-214, 1981.

[Robinson 1982] ROBINSON, S. M.; Generalized equations and their solutions, Part II: Applications to Nonlinear Programming. Mathematical Programming Studies, 19:200-221, 1982.

[Rockafellar 1970] ROCKAFELLAR, R.T.; Convex Analysis. Princeton University Press, 1970.

[Schläfli 1852] SCHLÄFLI, L.; Theorie der vielfachen Kontinuität (1852), pp 209-212. In.: Gesammelte Mathematische Abhandlungen I, Basel, Birkhäuser Verlag, 1950.

[Sherali \& Adams 1999] SHERALI, H.; ADAMS, W.; A ReformulationLinearization Technique for Solving Discrete and Continuous Nonconvex Problems, Kluwer Academic Press, 1999. 
[Sherali, Krishnamurty \& Al-Khayyal 1996] SHERALI, H.; KRISHNAMURTY, R. S.; AL-KHAYYAL, F. A.; Enhanced Intersection Cutting-Plane Approach for Linear Complementarity Problems. Journal of Optimization Theory and Applications, 90(1):183-201, 1996.

[Sherali, Krishnamurty \& Al-Khayyal 1998] SHERALI, H.; KRISHNAMURTY, R. S.; ALKHAYYAL, F. A.; Enumeration Approach for Linear Complementarity Problems Based on a Reformulation-Linearization Technique. Journal of Optimization Theory and Applications, 99(2):481-507, 1998.

[Spath 1992]

SPATH, H.; Mathematical Algorithms for Linear Regression. Academic Press, 1992.

[Stoer \& Burlisch 1992] STOER, J.; BURLISCH, R.; Introduction to Numerical Analysis. Springer Verlag, 1992.

[Stoer \& Witzgall 1970] STOER, J.; WITZGaLL, C.; Convexity and Optimization in Finite Dimensions. Springer Verlag, 1970.

[Thoai \& Tuy 1980] THOAI, N. V.; TUY, H.; Convergent Algorithms for Minimizing a Concave Function. Mathematics of Operations Research, 5(4):556-566, 1980.

[Thoai \& Tuy 1983] THOAI, N. V.; TUY, H.; Solving the Linear Complementarity Problem Through Concave Programming. U.S.S.R. Computati- 
[Tuy 1964]

[Tuy 1998]

[Wolfe 1962]

onal Mathematics and Mathematical Physics, 23(3):55-59, 1983.

TUY, H.; Concave Programming Under Linear Constraints. Soviet Mathematics Doklady, 5:1437-1440, 1964.

TUY, H.; Convex Analysis and Global Optimization. Kluwer Academic Publishers, 1998.

WOLFE, P.; The Reduced-Gradient Method, manuscrito não publicado, RAND Corporation, 1962.

[Ye \& Pardalos 1991]

YE, Y.; PARDALOS, P. M.; A Class of Linear Complementarity Problems Solvable in Polynomial Time. Linear Algebra and its Applications, 152:3-17, 1991.

[Ziegler 1995]

ZIEGLER, G. M.; Lectures on Polytopes. Springer-Verlag, 1995.

[Zwart 1973]

ZWART, P. B.; Nonlinear Programming: Counterexamples to 2 Global Optimization Algorithms. Operations Research, 21(6):12601266, 1973.

[Zwart 1974]

ZWART, P. B.; Global Maximization of a Convex Function with Linear Inequality Constraints. Operations Research, 22:602-609, 1974. 